Supporting Information

For the article entitled

\title{
Additive- and Ligand-Free Cross-Coupling Reactions between Alkenes and Alkynes by Iridium Catalysis
}

Yaling Sun, Keke Meng, Jian Zhang,* Mengxin Jin, Nini Huang, and Guofu Zhong*

Email: zhangjian@hznu.edu.cn; zgf@hznu.edu.cn

\section{Table of Contents}

General Methods

Optimization Conditions

SI-3

General Procedure for Catalytic Cross-Coupling Reactions

Characterization of Butadienes

SI-4

Gram-Scale Synthesis

SI-11

Deuterium-Labeled Experiments

SI-11

KIE Experiments

Competition Reaction

SI-15

Amide Group Removal

SI-16

References

SI-16

NMR Spectra

SI-17 


\section{General Methods}

Analytical thin layer chromatography (TLC) was performed using Merck 60 F254 precoated silica gel plate (0.2 mm thickness). Subsequent to elution, plates were visualized using UV radiation (254 nm) on Spectroline Model ENF-24061/F254 nm. Further visualization was possible by staining with basic solution of potassium permanganate or acidic solution of ceric molybdate. Flash column chromatography was performed using Merck aluminium oxide 90 active neutral with freshly distilled solvents. Columns were typically packed as slurry and equilibrated with the appropriate solvent system prior to use. Proton nuclear magnetic resonance spectra ( ${ }^{1} \mathrm{H}$ NMR) were recorded on Bruker AMX400 and $500 \mathrm{MHz}$ spectrophotometer $\left(\mathrm{CDCl}_{3}\right.$ as solvent). Chemical shifts for ${ }^{1} \mathrm{H}$ NMR spectra are reported as $\delta$ in units of parts per million (ppm) downfield from $\mathrm{SiMe}_{4}(\delta 0.0)$ and relative to the signal of chloroform-d ( $\delta 7.26$, singlet). Multiplicities were given as: s (singlet), d (doublet), t (triplet), dd (doublets of doublet) or m (multiplets). The number of protons (n) for a given resonance is indicated by nH. Coupling constants are reported as a $J$ value in Hz. Carbon nuclear magnetic resonance spectra $\left({ }^{13} \mathrm{C} \mathrm{NMR}\right)$ are reported as $\delta$ in units of parts per million (ppm) downfield from $\mathrm{SiMe}_{4}(\delta 0.0)$ and relative to the signal of chloroform- $d$ ( $\delta 77.0$, triplet). Mass spectrometry was performed by Waters Q-Tof Premier Micromass instrument, using Electro Spray Ionization (ESI) mode. IR spectra were recorded as thin films on $\mathrm{KBr}$ or $\mathrm{NaCl}$ plates on a Bio-Rad FTS 165 FTIR spectrometer and are reported in frequency of absorption $\left(\mathrm{cm}^{-1}\right)$. $\left[\mathrm{IrCp}^{*} \mathrm{Cl}_{2}\right]_{2},[\operatorname{Ir}(\operatorname{cod}) \mathrm{Cl}]_{2}$ and $\mathrm{AgBF}_{4}$ were purchased from Energy and used directly. Other reagents, unless otherwise noted below, are commercially available from Alfa Aesar (China) Chemical Co., Ltd. and used without further purification. Acrylamides were prepared by reported methods. $^{1-2}$ 
Table 1. Optimization Conditions for Alkene-Alkyne Coupling ${ }^{a}$
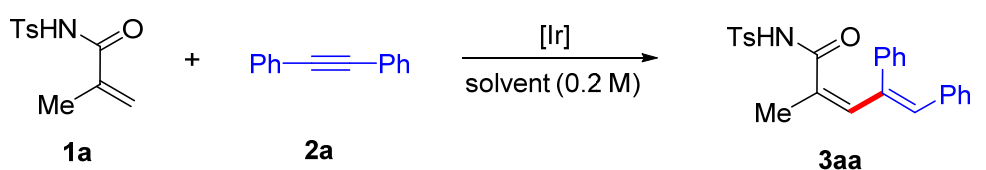

\begin{tabular}{|c|c|c|c|c|c|}
\hline Entry & Catalyst & Solvent & Temperature $\left({ }^{\circ} \mathrm{C}\right)$ & Time (h) & Yield $(\%)^{b}$ \\
\hline 1 & {$[\operatorname{IrCl}(\operatorname{cod})]_{2}$} & Toluene & 70 & 1.5 & 21 \\
\hline 2 & {$[\operatorname{IrCl}(\operatorname{cod})]_{2}$} & Toluene & 70 & 12 & 85 \\
\hline 3 & {$[\operatorname{IrCl}(\operatorname{cod})]_{2}$} & Toluene & 50 & 12 & 50 \\
\hline 4 & {$[\operatorname{IrCl}(\operatorname{cod})]_{2}$} & Toluene & 60 & 12 & 67 \\
\hline 5 & {$[\operatorname{IrCl}(\operatorname{cod})]_{2}$} & Toluene & 70 & 24 & 88 \\
\hline 6 & {$[\operatorname{IrOMe}(\operatorname{cod})]_{2}$} & Toluene & 70 & 24 & 74 \\
\hline 7 & {$[\operatorname{IrCl}(\operatorname{cod})]_{2}$} & 1,4-Dioxane & 70 & 24 & 86 \\
\hline 8 & {$[\operatorname{IrCl}(\operatorname{cod})]_{2}$} & DCE & 70 & 24 & 83 \\
\hline 9 & {$[\operatorname{IrCl}(\operatorname{cod})]_{2}$} & DME & 70 & 24 & 71 \\
\hline 10 & {$[\operatorname{IrCl}(\operatorname{cod})]_{2}$} & $\mathrm{MeOH}$ & 70 & 24 & 96 \\
\hline 11 & {$[\operatorname{IrCl}(\operatorname{cod})]_{2}$} & $\mathrm{EtOH}$ & 70 & 24 & 72 \\
\hline 12 & {$[\operatorname{IrCl}(\operatorname{cod})]_{2}$} & $i-\mathrm{PrOH}$ & 70 & 24 & 76 \\
\hline 13 & {$[\mathrm{IrCl}(\operatorname{cod})]_{2}$} & $\mathrm{CH}_{3} \mathrm{CH}_{2} \mathrm{OH}$ & 70 & 24 & 91 \\
\hline 14 & {$[\operatorname{IrCl}(\operatorname{cod})]_{2}$} & HFIP & 70 & 24 & 67 \\
\hline
\end{tabular}

${ }^{a}$ Conditions: 1a ( $0.2 \mathrm{mmol}, 1.0$ equiv), $2 \mathrm{a}(0.3 \mathrm{mmol}, 1.5$ equiv), [Ir] $(4 \mathrm{~mol} \%)$ in a solvent $(0.2 \mathrm{M})$ at $70{ }^{\circ} \mathrm{C}$ for $24 \mathrm{~h} .{ }^{b}$ Isolated yield. 


\section{General Procedure for Ir-Catalyzed Alkene-Alkyne Coupling Reaction}

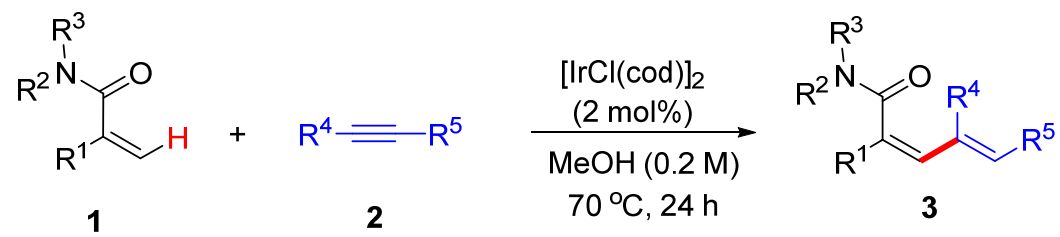

An oven-dried vial was charged with $[\operatorname{Ir}(\operatorname{cod}) \mathrm{Cl}]_{2}(2.7 \mathrm{mg}, 2 \mathrm{~mol} \%)$, and $\mathrm{MeOH}(1 \mathrm{~mL})$. Then, alkyne 2 ( $0.3 \mathrm{mmol}, 1.5$ equiv) and acrylamide 1 ( $0.2 \mathrm{mmol}, 1.0$ equiv) were added into the solution in sequence. The vial was sealed under argon and heated to $70{ }^{\circ} \mathrm{C}$ with stirring for 24 hours. After cooling down, the mixture was concentrated to give the crude product which was directly applied to a flash column chromatography for purification (EtOAc/Petroleum ether mixtures).

\section{Characterization of Butadienes}

(2Z, 4Z)-2-Methyl-4,5-diphenyl- $N$-tosylpenta-2,4-dienamide (3aa)<smiles>CCCCCNC(=O)/C(C)=C\C(=C\c1ccccc1)c1ccccc1</smiles>
Following the general experiment procedure, 3aa was obtained as a yellow solid $(96 \%, 38 \mathrm{mg})$. M. p. $=146{ }^{\circ} \mathrm{C} .{ }^{1} \mathrm{H}$ NMR $\left(500 \mathrm{MHz}, \mathrm{CDCl}_{3}\right): \delta=8.41$ $(\mathrm{s}, 1 \mathrm{H}), 7.60(\mathrm{~d}, J=8.5 \mathrm{~Hz}, 2 \mathrm{H}), 7.17-7.16(\mathrm{~m}, 3 \mathrm{H}), 7.08-7.02(\mathrm{~m}, 5 \mathrm{H}), 6.87$ $(\mathrm{d}, J=8.0 \mathrm{~Hz}, 2 \mathrm{H}), 6.79(\mathrm{~d}, J=7.0 \mathrm{~Hz}, 2 \mathrm{H}), 6.32(\mathrm{~s}, 1 \mathrm{H}), 6.25(\mathrm{t}, J=1.5 \mathrm{~Hz}$, 1H), $2.20(\mathrm{~s}, 3 \mathrm{H}), 1.93(\mathrm{~d}, J=1.5 \mathrm{~Hz}, 3 \mathrm{H}) .{ }^{13} \mathrm{C} \mathrm{NMR}\left(125 \mathrm{MHz}, \mathrm{CDCl}_{3}\right): \delta=166.02,143.66,136.71$, $135.77,135.36,134.82,133.84,131.20,131.05,128.65,128.25,128.12,127.78,127.38,127.16$, 126.99, 126.59, 20.61, 20.09. HR-MS (ESI): m/z calculated for $\mathrm{C}_{25} \mathrm{H}_{23} \mathrm{NO}_{3} \mathrm{~S}:[\mathrm{M}+\mathrm{H}]^{+}: 418.1471$, found: 418.1475. FTIR (KBr, $\left.\mathrm{cm}^{-1}\right): 3685.35,3419.37,2974.61,2899.50,1651.53,1384.18,1087.13$, 1048.10.

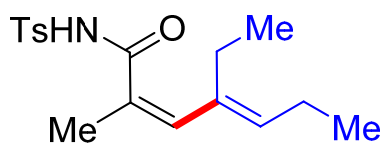

\section{(2Z, 4E)-4-Ethyl-2-methyl- $N$-tosylhepta-2,4-dienamide (3ab)}

Following the general experiment procedure, 3ab was obtained as a yellow liquid (69\%, $44 \mathrm{mg}) .{ }^{1} \mathrm{H}$ NMR (500 MHz, $\left.\mathrm{CDCl}_{3}\right): \delta=8.61(\mathrm{~s}$, $1 \mathrm{H}), 7.95(\mathrm{~d}, J=8.5 \mathrm{~Hz}, 2 \mathrm{H}), 7.33(\mathrm{~d}, J=8.0 \mathrm{~Hz}, 2 \mathrm{H}), 6.31(\mathrm{~s}, 1 \mathrm{H}), 5.40(\mathrm{t}, J=7.0 \mathrm{~Hz}, 1 \mathrm{H}), 2.44$ (s, $3 \mathrm{H}), 2.17-2.05(\mathrm{~m}, 4 \mathrm{H}), 1.88(\mathrm{~d}, J=1.5 \mathrm{~Hz}, 3 \mathrm{H}), 1.00(\mathrm{td}, J=7.5,4.5 \mathrm{~Hz}, 6 \mathrm{H}) .{ }^{13} \mathrm{C}$ NMR $(125 \mathrm{MHz}$, $\left.\mathrm{CDCl}_{3}\right): \delta=165.06,143.88,137.64,136.42,134.82,133.74,128.66,128.47,127.47,22.52,20.64$, 20.29, 19.55, 12.67, 12.35. HR-MS (ESI): $\mathrm{m} / \mathrm{z}$ calculated for $\mathrm{C}_{17} \mathrm{H}_{23} \mathrm{NO}_{3} \mathrm{~S}:[\mathrm{M}+\mathrm{H}]^{+}: 322.1471$, found: 322.1473. FTIR (KBr, $\left.\mathrm{cm}^{-1}\right): 3850.93,3646.34,3500.35,3444.47,1633.98,1384.26,1164.90$, 1083.37 .<smiles>CC=C(C)C=C(C)C(=O)NCCCCC</smiles>

(2Z, 4E)-2, 4-Dimethyl- $N$-tosylhexa-2,4-dienamide (3ac)

Following the general experiment procedure, 3ac was obtained as a yellow liquid $(77 \%, 45 \mathrm{mg}) .{ }^{1} \mathrm{H}$ NMR $\left(500 \mathrm{MHz}, \mathrm{CDCl}_{3}\right): \delta=8.24(\mathrm{~s}, 1 \mathrm{H}), 7.88(\mathrm{~d}$, $J=8.5 \mathrm{~Hz}, 2 \mathrm{H}), 7.27(\mathrm{~d}, J=8.0 \mathrm{~Hz}, 2 \mathrm{H}), 6.12(\mathrm{~s}, 1 \mathrm{H}), 5.43(\mathrm{~d}, J=6.5 \mathrm{~Hz}, 1 \mathrm{H}), 2.37$ (s, 3H), $1.80(\mathrm{~d}, J$ $=1.5 \mathrm{~Hz}, 3 \mathrm{H}), 1.57(\mathrm{~s}, 3 \mathrm{H}), 1.56(\mathrm{~d}, J=6.0 \mathrm{~Hz}, 3 \mathrm{H}) .{ }^{13} \mathrm{C} \mathrm{NMR}\left(125 \mathrm{MHz}, \mathrm{CDCl}_{3}\right): \delta=165.95,144.01$, 
137.62, 134.52, 131.23, 128.49, 127.99, 127.54, 127.07, 20.60, 19.74, 14.42, 12.90. HR-MS (ESI): m/z calculated for $\mathrm{C}_{15} \mathrm{H}_{19} \mathrm{NO}_{3} \mathrm{~S}:[\mathrm{M}+\mathrm{H}]^{+}: 294.1158$, found: 294.1157. FTIR (KBr, cm$\left.{ }^{-1}\right)$ : 3627.22, 3444.29, $2980.82,1668.18,1557.87,1384.26,1164.45,1082.29$.

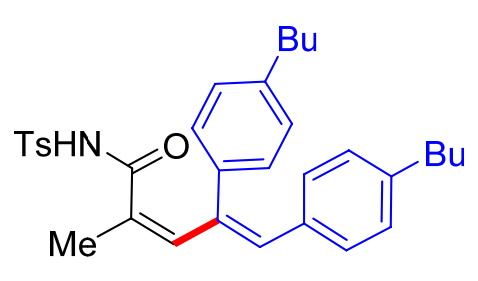

(2Z,4Z)-4,5-bis(4-Butylphenyl)-2-methyl- $N$-tosylpenta-2,4-diena mide (3ad).

Following the general experiment procedure, 3ad was obtained as a yellow solid $(84 \%, 89 \mathrm{mg})$. M. p. $=92{ }^{\circ} \mathrm{C} .{ }^{1} \mathrm{H}$ NMR $(500 \mathrm{MHz}$, $\left.\mathrm{CDCl}_{3}\right): \delta=7.65(\mathrm{~d}, J=8.5 \mathrm{~Hz}, 2 \mathrm{H}), 7.05-7.09(\mathrm{~m}, 4 \mathrm{H}), 6.96-6.92$ $(\mathrm{dd}, J=12.0,8.5 \mathrm{~Hz}, 4 \mathrm{H}), 6.83(\mathrm{~d}, J=8.0 \mathrm{~Hz}, 2 \mathrm{H}), 6.36(\mathrm{~d}, J=1.5 \mathrm{~Hz}, 1 \mathrm{H}), 6.33(\mathrm{~s}, 1 \mathrm{H}), 2.61-2.55$ (m, 4H), 2.29 (s, 3H), $1.99(\mathrm{~d}, J=1.5 \mathrm{~Hz}, 3 \mathrm{H}), 1.62-1.56(\mathrm{~m}, 4 \mathrm{H}), 1.39-1.34(\mathrm{~m}, 4 \mathrm{H}), 0.94(\mathrm{td}, J=7.5$, $2.5 \mathrm{~Hz}, 6 \mathrm{H}) .{ }^{13} \mathrm{C} \mathrm{NMR}\left(125 \mathrm{MHz}, \mathrm{CDCl}_{3}\right): \delta=166.03,143.48,142.04,141.60,136.22,134.82,134.00$, 133.97, 132.31, 130.83, 130.67, 128.62, 128.18, 127.93, 127.83, 127.44, 127.03, 34.42, 34.38, 32.45, $32.39,21.42,21.33,20.59,19.98,12.94,12.93$. HR-MS (ESI): $\mathrm{m} / \mathrm{z}$ calculated for $\mathrm{C}_{33} \mathrm{H}_{39} \mathrm{NO}_{3} \mathrm{~S}$ : $[\mathrm{M}+\mathrm{H}]^{+}:$530.2723, found: 530.2731. FTIR $\left(\mathrm{KBr}, \mathrm{cm}^{-1}\right): 3646.52,3444.43,2956.09,2923.57,1651.68$, $1384.19,1163.79,1082.07$.

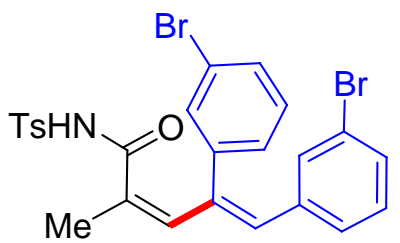

(2Z,4Z)-4,5-bis(3-Bromophenyl)-2-methyl- $N$-tosylpenta-2,4-dienami de (3ae).

Following the general experiment procedure, 3ae was obtained as a white solid (90\%, $83 \mathrm{mg})$. M. p. $=206^{\circ} \mathrm{C} .{ }^{1} \mathrm{H} \mathrm{NMR}\left(500 \mathrm{MHz}, \mathrm{CDCl}_{3}\right)$ : $\delta=8.45(\mathrm{~s}, 1 \mathrm{H}), 7.73(\mathrm{~d}, J=8.0 \mathrm{~Hz}, 2 \mathrm{H}), 7.36(\mathrm{ddd}, J=3.0,1.5,1.0 \mathrm{~Hz}, 1 \mathrm{H}), 7.31-7.29(\mathrm{~m}, 1 \mathrm{H}), 7.26$ (s, 1H), $7.09(\mathrm{t}, J=7.5 \mathrm{~Hz}, 1 \mathrm{H}), 7.01-7.03(\mathrm{~m}, 3 \mathrm{H}), 6.99-6.96(\mathrm{~m}, 2 \mathrm{H}), 6.75(\mathrm{~d}, J=7.5 \mathrm{~Hz}, 1 \mathrm{H}), 6.31(\mathrm{~s}$, $1 \mathrm{H}), 6.20(\mathrm{t}, J=1.5 \mathrm{~Hz}, 1 \mathrm{H}), 2.31(\mathrm{~s}, 3 \mathrm{H}), 2.05-2.04(\mathrm{t}, J=1.5 \mathrm{~Hz}, 3 \mathrm{H}) .{ }^{13} \mathrm{C} \mathrm{NMR}\left(125 \mathrm{MHz}, \mathrm{CDCl}_{3}\right)$ : $\delta=165.97,144.03,138.49,136.58,135.82,133.69,133.44,132.65,131.54,130.92,130.39,129.62$, 129.60, 129.23, 128.48, 128.32, 127.31, 126.94, 126.91, 121.78, 121.14, 20.68, 20.21. HR-MS (ESI): $\mathrm{m} / \mathrm{z}$ calculated for $\mathrm{C}_{25} \mathrm{H}_{23} \mathrm{Br}_{2} \mathrm{NO}_{3} \mathrm{~S}$ : $[\mathrm{M}+\mathrm{H}]^{+}:$575.9838, found: 575.9843. FTIR (KBr, $\left.\mathrm{cm}^{-1}\right)$ : 3626.43, $3564.48,3472.97,3287.26,2932.48,1651.80,1384.21,480.23$.

\section{(2Z, 4Z)-2-Methyl-4-phenyl- $N$-tosylhexa-2,4-dienamide (3af)}

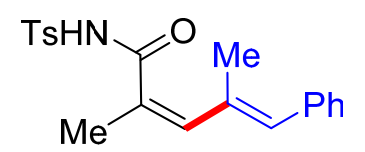

Following the general experiment procedure, 3af was obtained as a white solid (51\%, $38 \mathrm{mg})$. M. p. $=153{ }^{\circ} \mathrm{C} .{ }^{1} \mathrm{H}$ NMR (500 MHz, $\left.\mathrm{CDCl}_{3}\right): \delta=8.31(\mathrm{~s}$, 1H), 7.73 (d, $J=8.0 \mathrm{~Hz}, 2 \mathrm{H}), 7.27$ (t, $J=7.5 \mathrm{~Hz}, 2 \mathrm{H}), 7.21-7.19(\mathrm{~m}, 1 \mathrm{H})$, 7.08 (d, $J=7.5 \mathrm{~Hz}, 2 \mathrm{H}), 6.99$ (d, $J=8.0 \mathrm{~Hz}, 2 \mathrm{H}), 6.21(\mathrm{~s}, 1 \mathrm{H}), 6.17(\mathrm{~s}, 1 \mathrm{H}), 2.25$ (s, 3H), 1.90 (d, $J=$ $1.0 \mathrm{~Hz}, 3 \mathrm{H}), 1.82(\mathrm{~s}, 3 \mathrm{H}) .{ }^{13} \mathrm{C}$ NMR $\left(125 \mathrm{MHz}, \mathrm{CDCl}_{3}\right): \delta=166.36,143.89,137.07,135.54,133.87$, $131.99,131.62,129.05,128.34,128.25,127.47,127.18,126.28,20.63,20.15,16.69$. HR-MS (ESI): $\mathrm{m} / \mathrm{z}$ calculated for $\mathrm{C}_{20} \mathrm{H}_{21} \mathrm{NO}_{3} \mathrm{~S}:[\mathrm{M}+\mathrm{H}]^{+}:$356.1315, found: 356.1314. FTIR $\left(\mathrm{KBr}, \mathrm{cm}^{-1}\right)$ : 3626.10, $3573.43,3450.95,1645.11,1557.59,1384.17,1163.73,1083.16$.<smiles>[CH2]NC(=O)/C(=C\C=C(CCCCC)CCCCC)c1ccccc1</smiles>

(2Z,4E)-4-Pentyl-2-phenyl- $N$-tosyldeca-2,4-dienamide (3bg).

Following the general experiment procedure, $3 \mathbf{b g}$ was obtained as a yellow solid $(93 \%, 88 \mathrm{mg})$. M. p. $=237^{\circ} \mathrm{C} .{ }^{1} \mathrm{H}$ NMR $(500$ 
$\left.\mathrm{MHz}, \mathrm{CDCl}_{3}\right): \delta=8.17(\mathrm{~s}, 1 \mathrm{H}), 7.87(\mathrm{~d}, J=8.0 \mathrm{~Hz}, 2 \mathrm{H}), 7.25(\mathrm{~d}, J=8.0 \mathrm{~Hz}, 2 \mathrm{H}), 7.18-7.16(\mathrm{~m}, 3 \mathrm{H})$, 7.11-7.09 (m, 2H), $6.38(\mathrm{~s}, 1 \mathrm{H}), 5.47(\mathrm{t}, J=7.5 \mathrm{~Hz}, 1 \mathrm{H}), 2.37(\mathrm{~s}, 3 \mathrm{H}), 2.00((\mathrm{t}, J=8.0 \mathrm{~Hz}, 2 \mathrm{H})$, 1.84-1.79 (m, 2H), 1.23-1.12 (m, 12H), 0.83-0.79 (m, 6H). $\left.{ }^{13} \mathrm{C} \mathrm{NMR} \mathrm{(125} \mathrm{MHz,} \mathrm{CDCl}_{3}\right): \delta=165.68$, 144.02, 135.35, 135.28, 134.97, 134.54, 134.44, 132.27, 128.49, 127.70, 127.63, 127.21, 125.43, 35.75, 30.67, 30.66, 28.86, 27.86, 27.84, 27.34, 21.49, 21.47, 20.67, 13.02. HR-MS (ESI): $\mathrm{m} / \mathrm{z}$ calculated for $\mathrm{C}_{28} \mathrm{H}_{37} \mathrm{NO}_{3} \mathrm{~S}:[\mathrm{M}+\mathrm{H}]^{+}: 468.2567$, found: 468.2562. FTIR $\left(\mathrm{KBr}, \mathrm{cm}^{-1}\right): 3850.92,3534.17,3444.62$, 2923.03, 2330.28, 1644.97, 1455.37, 1384.19 .

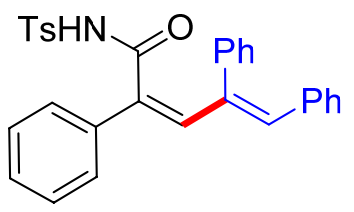

(2Z, 4Z)-2, 4, 5-Triphenyl- $N$-tosylpenta-2, 4-dienamide (3ba).

Following the general experiment procedure, 3 ba was obtained as a yellow solid $(84 \%, 80 \mathrm{mg})$. M. p. $=221^{\circ} \mathrm{C} .{ }^{1} \mathrm{H}$ NMR $\left(500 \mathrm{MHz}, \mathrm{CDCl}_{3}\right): \delta=7.71$ $(\mathrm{d}, J=8.0 \mathrm{~Hz}, 2 \mathrm{H}), 7.30-7.27(\mathrm{~m}, 5 \mathrm{H}), 7.22-7.20(\mathrm{~m}, 3 \mathrm{H}), 7.17-7.15(\mathrm{~m}$, 2H), 7.13-7.10 (dt, $J=7.0,2.5 \mathrm{~Hz}, 1 \mathrm{H}), 7.08-7.05(\mathrm{~m}, 2 \mathrm{H}), 6.93(\mathrm{~d}, J=8.0 \mathrm{~Hz}, 2 \mathrm{H}), 6.80(\mathrm{~d}, J=7.0$ $\mathrm{Hz}, 2 \mathrm{H}), 6.67(\mathrm{~d}, J=1.0 \mathrm{~Hz}, 1 \mathrm{H}), 6.62(\mathrm{~s}, 1 \mathrm{H}), 2.25(\mathrm{~s}, 3 \mathrm{H}) .{ }^{13} \mathrm{C} \mathrm{NMR}\left(125 \mathrm{MHz}, \mathrm{CDCl}_{3}\right): \delta=165.38$, $143.67,137.13,136.60,134.97,134.92,134.83,133.82,133.78,131.51,128.75,128.39,128.24$, 127.83, 127.66, 127.28, 126.97, 126.82, 126.40, 125.54, 20.63. HR-MS (ESI): $\mathrm{m} / \mathrm{z}$ calculated for $\mathrm{C}_{30} \mathrm{H}_{25} \mathrm{NO}_{3} \mathrm{~S}:[\mathrm{M}+\mathrm{H}]^{+}: 480.1628$, found: 480.1623. FTIR $\left(\mathrm{KBr}, \mathrm{cm}^{-1}\right): 3850.98,3626.64,3444.57$, 2930.42, 2357.26, 1651.64, 1384.28, 683.22.<smiles>COc1ccc(C(=CC(=Cc2ccccc2)C(=O)N[TeH])c2ccccc2)cc1</smiles>

(2Z,4Z)-2-(4-Methoxylphenyl)-4,5-diphenyl- $N$-tosylpenta-2,4-di enamide (3ca).

Following the general experiment procedure, 3ca was obtained as a yellow solid $(74 \%, 75 \mathrm{mg})$. M. p. $=225{ }^{\circ} \mathrm{C} .{ }^{1} \mathrm{H}$ NMR $(500$ $\left.\mathrm{MHz}, \mathrm{CDCl}_{3}\right): \delta=8.62(\mathrm{~s}, 1 \mathrm{H}), 7.61(\mathrm{~d}, J=8.5 \mathrm{~Hz}, 2 \mathrm{H}), 7.15-7.11(\mathrm{~m}, 5 \mathrm{H}), 7.07(\mathrm{dd}, J=7.5,3.5 \mathrm{~Hz}$, 2H), $7.02(\mathrm{~d}, J=7.5 \mathrm{~Hz}, 1 \mathrm{H}), 6.97(\mathrm{t}, J=7.5 \mathrm{~Hz}, 2 \mathrm{H}), 6.82(\mathrm{~d}, J=8.5 \mathrm{~Hz}, 2 \mathrm{H}), 6.70(\mathrm{t}, J=8.5 \mathrm{~Hz}, 4 \mathrm{H})$, $6.49(\mathrm{~s}, 1 \mathrm{H}), 6.47(\mathrm{~d}, J=1.5 \mathrm{~Hz}, 1 \mathrm{H}), 3.68(\mathrm{~s}, 3 \mathrm{H}), 2.15(\mathrm{~s}, 3 \mathrm{H}) .{ }^{13} \mathrm{C}$ NMR $\left(125 \mathrm{MHz}, \mathrm{CDCl}_{3}\right): \delta=$ $165.63,159.01,143.63,137.35,136.74,135.11,134.57,133.79,131.95,130.88,128.71,128.41$, 128.22, 127.61, 127.34, 127.26, 126.93, 126.90, 126.79, 126.26, 113.26, 54.31, 20.62. HR-MS (ESI): $\mathrm{m} / \mathrm{z}$ calculated for $\mathrm{C}_{31} \mathrm{H}_{27} \mathrm{NO}_{4} \mathrm{~S}:[\mathrm{M}+\mathrm{H}]^{+}: 510.1734$, found: 510.1731 . FTIR $\left(\mathrm{KBr}, \mathrm{cm}^{-1}\right): 3876.56$, $3564.33,3444.34,2382.40,1714.31,1633.92,1434.65,1360.44$.<smiles>O=C(N[TeH])C(=CC(=Cc1ccccc1)c1ccccc1)c1ccccc1</smiles>

(2Z,4Z)-2-(4-Trifluoromethylphenyl)-4,5-diphenyl- $N$-tosylpenta2,4-dienamide (3da).

Following the general experiment procedure, 3da was obtained as a yellow solid $(86 \%, 94 \mathrm{mg})$. M. p. $=215^{\circ} \mathrm{C} .{ }^{1} \mathrm{H}$ NMR $(500 \mathrm{MHz}$, $\left.\mathrm{CDCl}_{3}\right): \delta=8.65(\mathrm{~s}, 1 \mathrm{H}), 7.67(\mathrm{~d}, J=8.0 \mathrm{~Hz}, 2 \mathrm{H}), 7.52(\mathrm{~d}, J=8.0 \mathrm{~Hz}, 2 \mathrm{H}), 7.39(\mathrm{~d}, J=8.0 \mathrm{~Hz}, 2 \mathrm{H})$, 7.25-7.23 (m, 3H), 7.18-7.15 (m, 3H), $7.09(\mathrm{t}, J=7.5 \mathrm{~Hz}, 2 \mathrm{H}), 6.95(\mathrm{~d}, J=8.0 \mathrm{~Hz}, 2 \mathrm{H}), 6.81(\mathrm{~d}, J=7.5$ $\mathrm{Hz}, 2 \mathrm{H}), 6.77(\mathrm{~d}, J=1.0 \mathrm{~Hz}, 1 \mathrm{H}), 6.68(\mathrm{~s}, 1 \mathrm{H}), 2.27(\mathrm{~s}, 3 \mathrm{H}) .{ }^{13} \mathrm{C} \mathrm{NMR}\left(125 \mathrm{MHz}, \mathrm{CDCl}_{3}\right): \delta=164.78$, $143.97,138.37,136.66,136.21,135.81,134.66,133.56,133.40,132.89,128.82,128.38,128.32$, $127.81,127.24,126.93,126.79,126.52,126.33,125.78,124.74\left(J_{C-F}=3.8 \mathrm{~Hz}\right), 122.86\left(J_{C-F}=270.4\right.$ $\mathrm{Hz}$ ), 20.61. HR-MS (ESI): $\mathrm{m} / \mathrm{z}$ calculated for $\mathrm{C}_{31} \mathrm{H}_{24} \mathrm{FNO}_{3} \mathrm{~S}:[\mathrm{M}+\mathrm{H}]^{+}: 548.1502$, found: 548.1496 . FTIR $\left(\mathrm{KBr}, \mathrm{cm}^{-1}\right)$ : 3850.02, 3507.34, 3444.48, 2956.12, 1651.57, 1463.05, 1123.78, 1083.92. 
<smiles>O=C(N[Ga])/C(=C\C(=C/c1ccccc1)c1ccc(F)cc1)c1ccccc1</smiles>

(2Z,4Z)-2-(4-Fluorophenyl)-4,5-diphenyl- $N$-tosylpenta-2,4-dienamid e (3ea).

Following the general experiment procedure, 3ea was obtained as a yellow solid $(98 \%, 98 \mathrm{mg})$. M. p. $=180{ }^{\circ} \mathrm{C} .{ }^{1} \mathrm{H}$ NMR $(500 \mathrm{MHz}$, $\left.\mathrm{CDCl}_{3}\right): \delta=8.49(\mathrm{~s}, 1 \mathrm{H}), 7.69(\mathrm{~d}, J=8.0 \mathrm{~Hz}, 2 \mathrm{H}), 7.29-7.23(\mathrm{~m}, 5 \mathrm{H}), 7.17-7.12(\mathrm{~m}, 3 \mathrm{H}), 7.08(\mathrm{t}, J=7.5$ $\mathrm{Hz}, 2 \mathrm{H}), 6.99-6.93(\mathrm{~m}, 4 \mathrm{H}), 6.80(\mathrm{~d}, J=7.5 \mathrm{~Hz}, 2 \mathrm{H}), 6.62$ (d, $J=13.0 \mathrm{~Hz}, 2 \mathrm{H}), 2.26(\mathrm{~s}, 3 \mathrm{H}) .{ }^{13} \mathrm{C} \mathrm{NMR}$ $\left(125 \mathrm{MHz}, \mathrm{CDCl}_{3}\right): \delta=165.15,161.92\left(J_{C-F}=248.0 \mathrm{~Hz}\right), 160.93,143.84,136.97,136.38,134.84$, $133.87\left(J_{C-F}=4.6 \mathrm{~Hz}\right), 133.63,131.04\left(J_{C-F}=3.3 \mathrm{~Hz}\right), 128.75,128.35,128.28,127.74,127.48,127.42$, 127.29, 127.09, 126.87, 126.54, $114.86\left(J_{C-F}=21.6 \mathrm{~Hz}\right), 20.63$. HR-MS (ESI): $\mathrm{m} / \mathrm{z}$ calculated for $\mathrm{C}_{30} \mathrm{H}_{24} \mathrm{FNO}_{3} \mathrm{~S}:[\mathrm{M}+\mathrm{H}]^{+}:$498.1534, found: 498.1531. FTIR ( $\left.\mathrm{KBr}, \mathrm{cm}^{-1}\right)$ : 3654.91, 3550.29, 3444.57, 2983.70, 1651.68, 1538.91, 1384.31, 470.37 .<smiles>O=C(N[AlH2])C(=CC(=Cc1ccccc1)c1ccccc1F)c1ccccc1</smiles>

(2Z,4Z)-2-(2-Fluorophenyl)-4,5-diphenyl- $N$-tosylpenta-2,4-dienamide (3fa).

Following the general experiment procedure, 3fa was obtained as a yellow solid $(75 \%, 76 \mathrm{mg}) . \mathrm{M} . \mathrm{p} .=230{ }^{\circ} \mathrm{C} .{ }^{1} \mathrm{H} \mathrm{NMR}\left(500 \mathrm{MHz}, \mathrm{CDCl}_{3}\right): \delta=8.71$ $(\mathrm{s}, 1 \mathrm{H}), 7.73(\mathrm{~d}, J=8.0 \mathrm{~Hz}, 2 \mathrm{H}), 7.29-7.26(\mathrm{~m}, 2 \mathrm{H}), 7.23-7.20(\mathrm{~m}, 5 \mathrm{H}), 7.14(\mathrm{~d}, J=7.0 \mathrm{~Hz}, 1 \mathrm{H})$, 7.10-7.06 (m, 3H), $7.00(\mathrm{td}, J=8.5,0.5 \mathrm{~Hz}, 1 \mathrm{H}), 6.94(\mathrm{~d}, J=8.0 \mathrm{~Hz}, 2 \mathrm{H}), 6.87(\mathrm{~d}, J=7.5 \mathrm{~Hz}, 2 \mathrm{H})$, $6.69(\mathrm{~d}, J=1.5 \mathrm{~Hz}, 1 \mathrm{H}), 6.63(\mathrm{~s}, 1 \mathrm{H}), 2.25(\mathrm{~s}, 3 \mathrm{H}) .{ }^{13} \mathrm{C} \mathrm{NMR}\left(125 \mathrm{MHz}, \mathrm{CDCl}_{3}\right): \delta=164.58,158.91$ $\left(J_{C-F}=248.0 \mathrm{~Hz}\right), 143.56,138.24\left(J_{C-F}=2.8 \mathrm{~Hz}\right), 136.85,136.02,134.84,133.82,132.05,130.01$, $129.55\left(J_{C-F}=8.4 \mathrm{~Hz}\right), 129.33\left(J_{C-F}=2.7 \mathrm{~Hz}\right), 128.83,128.32,128.17,127.70,127.33,127.04,126.88$, 126.62, $123.86\left(J_{C-F}=13.2 \mathrm{~Hz}\right), 123.51\left(J_{C-F}=3.5 \mathrm{~Hz}\right), 114.98\left(J_{C-F}=21.8 \mathrm{~Hz}\right), 20.61$. HR-MS (ESI): $\mathrm{m} / \mathrm{z}$ calculated for $\mathrm{C}_{30} \mathrm{H}_{24} \mathrm{FNO}_{3} \mathrm{~S}:[\mathrm{M}+\mathrm{H}]^{+}:$498.1534, found: 498.1538. FTIR (KBr, $\left.\mathrm{cm}^{-1}\right): 3829.87$, $3520.45,3444.99,2980.17,1698.65,1682.47,1384.44,1084.11$.

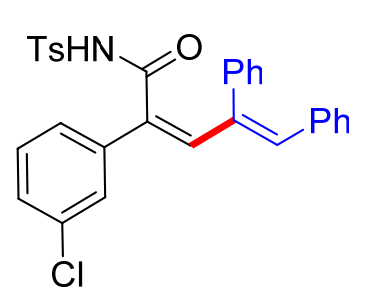

(2Z,4Z)-2-(2-Chlorophenyl)-4,5-diphenyl- $N$-tosylpenta-2,4-dienamid e (3ga)

Following the general experiment procedure, 3ga was obtained as a yellow solid $(67 \%, 69 \mathrm{mg}) . \mathrm{M} . \mathrm{p} .=171^{\circ} \mathrm{C} .{ }^{1} \mathrm{H}$ NMR $(500 \mathrm{MHz}$, $\left.\mathrm{CDCl}_{3}\right): \delta=8.60(\mathrm{~s}, 1 \mathrm{H}), 7.70(\mathrm{~d}, J=8.0 \mathrm{~Hz}, 2 \mathrm{H}), 7.25-7.23(\mathrm{~m}, 4 \mathrm{H})$, 7.20-7.12 (m, 6H), 7.08 (t, $J=7.5 \mathrm{~Hz}, 2 \mathrm{H}), 6.98(\mathrm{~d}, J=8.5 \mathrm{~Hz}, 2 \mathrm{H}), 6.82(\mathrm{~d}, J=7.5 \mathrm{~Hz}, 2 \mathrm{H}), 6.70(\mathrm{~d}, J$ $=1.0 \mathrm{~Hz}, 1 \mathrm{H}), 6.66(\mathrm{~s}, 1 \mathrm{H}), 2.28(\mathrm{~s}, 3 \mathrm{H}) .{ }^{13} \mathrm{C} \mathrm{NMR}\left(125 \mathrm{MHz}, \mathrm{CDCl}_{3}\right): \delta=164.75,143.91,136.73$, $136.72,136.32,135.10,134.76,133.73,133.59,133.38,132.62,129.03,128.79,128.38,128.37$, 127.80, 127.63, 127.23, 127.18, 126.90, 126.66, 125.39, 123.74, 20.66. HR-MS (ESI): m/z calculated for $\mathrm{C}_{30} \mathrm{H}_{24} \mathrm{ClNO}_{3} \mathrm{~S}:[\mathrm{M}+\mathrm{H}]^{+}:$514.1238, found: 514.1239. FTIR (KBr, cm $\left.{ }^{-1}\right): 3813.80,3616.82,3444.55$, 2980.34, 2330.23, 1694.40, 1557.40, 1384.28.

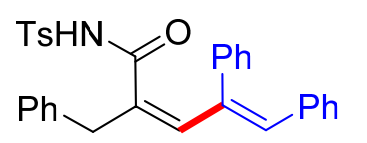

(2Z, 4Z)-2-Benzyl-4,5-diphenyl- $N$-tosylpenta-2,4-dienamide (3ha).

Following the general experiment procedure, 3ha was obtained as a yellow solid $(81 \%, 80 \mathrm{mg})$. M. p. $=176{ }^{\circ} \mathrm{C} .{ }^{1} \mathrm{H}$ NMR $\left(500 \mathrm{MHz}, \mathrm{CDCl}_{3}\right): \delta=8.40(\mathrm{~s}, 1 \mathrm{H}), 7.59(\mathrm{~d}, J$ $=8.5 \mathrm{~Hz}, 2 \mathrm{H}), 7.28(\mathrm{~s}, 1 \mathrm{H}), 7.22-7.19(\mathrm{~m}, 5 \mathrm{H}), 7.13-7.08(\mathrm{~m}, 7 \mathrm{H}), 6.93(\mathrm{~d}, J=8.0 \mathrm{~Hz}, 2 \mathrm{H}), 6.86(\mathrm{~d}, J=$ 
$7.0 \mathrm{~Hz}, 2 \mathrm{H}), 6.49(\mathrm{~s}, 1 \mathrm{H}), 6.25(\mathrm{~s}, 1 \mathrm{H}), 3.61(\mathrm{~s}, 2 \mathrm{H}), 2.28(\mathrm{~s}, 3 \mathrm{H}) \cdot{ }^{13} \mathrm{C} \mathrm{NMR}\left(125 \mathrm{MHz}, \mathrm{CDCl}_{3}\right): \delta=$ $165.84,143.47,136.76,136.16,135.72,135.11,135.05,134.92,133.88,131.32,128.63,128.19$, 128.16, 127.91, 127.75, 127.72, 127.21, 127.06, 126.91, 126.48, 125.77, 40.15, 20.59. HR-MS (ESI): $\mathrm{m} / \mathrm{z}$ calculated for $\mathrm{C}_{31} \mathrm{H}_{27} \mathrm{NO}_{3} \mathrm{~S}:[\mathrm{M}+\mathrm{H}]^{+}:$494.1784, found: 494.1777. FTIR $\left(\mathrm{KBr}, \mathrm{cm}^{-1}\right)$ : 3851.07, 3606.10, 3472.55, 2922.66, 1698.63, 1574.44, 1384.20, 1157.74 .

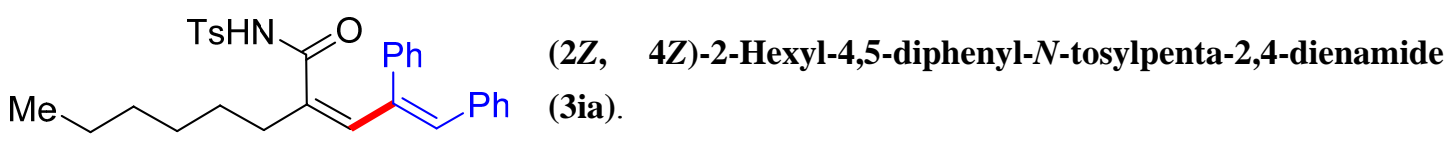
Following the general experiment procedure, 3ia was obtained as a yellow solid $(88 \%, 89 \mathrm{mg})$. M. p. $=146{ }^{\circ} \mathrm{C} .{ }^{1} \mathrm{H}$ NMR $\left(500 \mathrm{MHz}, \mathrm{CDCl}_{3}\right): \delta=8.53(\mathrm{~s}, 1 \mathrm{H}), 7.69(\mathrm{~d}$, $J=8.0 \mathrm{~Hz}, 2 \mathrm{H}), 7.22(\mathrm{dd}, J=6.0,2.5 \mathrm{~Hz}, 3 \mathrm{H}), 7.15-7.12(\mathrm{~m}, 3 \mathrm{H}), 7.08(\mathrm{t}, J=7.5 \mathrm{~Hz}, 2 \mathrm{H}), 6.91(\mathrm{~d}, J=$ $8.0 \mathrm{~Hz}, 2 \mathrm{H}), 6.83(\mathrm{~d}, J=7.5 \mathrm{~Hz}, 2 \mathrm{H}), 6.39(\mathrm{~s}, 1 \mathrm{H}), 6.22(\mathrm{~s}, 1 \mathrm{H}), 2.34-2.29(\mathrm{t}, J=7.0 \mathrm{~Hz}, 2 \mathrm{H}), 2.26$ (s, $3 \mathrm{H}), 1.45-1.39(\mathrm{~m}, 2 \mathrm{H}), 1.28-1.23(\mathrm{~m}, 6 \mathrm{H}), 0.86(\mathrm{t}, J=7.0 \mathrm{~Hz}, 3 \mathrm{H}) .{ }^{13} \mathrm{C}$ NMR $\left(125 \mathrm{MHz}, \mathrm{CDCl}_{3}\right): \delta=$ $166.47,143.57,137.06,136.20,135.90,135.04,133.82,133.71,130.56,128.65,128.20,127.70$, 127.32, 127.01, 126.88, 126.37, 125.76, 34.40, 30.45, 27.76, 27.01, 21.50, 20.59, 13.03. HR-MS (ESI): $\mathrm{m} / \mathrm{z}$ calculated for $\mathrm{C}_{30} \mathrm{H}_{34} \mathrm{NO}_{3} \mathrm{~S}:[\mathrm{M}+\mathrm{H}]^{+}: 488.2254$, found: 488.2247. FTIR $\left(\mathrm{KBr}, \mathrm{cm}^{-1}\right)$ : 3667.30 , $3605.72,3450.97,2950.32,1651.72,1505.05,1384.23,449.35$.<smiles>CCCCCNC(=O)C(=Cc1ccccc1)C=C(CCCCC)C(=O)NCC</smiles>

(2Z,4Z)-2-Decyl-4,5-diphenyl- $N$-tosylpenta-2,4-dienamide (3ja).

Following the general experiment procedure, $3 \mathbf{j a}$ was obtained as a yellow solid $(89 \%, 97 \mathrm{mg})$. M. p. $=180{ }^{\circ} \mathrm{C} .{ }^{1} \mathrm{H} \operatorname{NMR}\left(500 \mathrm{MHz}, \mathrm{CDCl}_{3}\right): \delta=8.61(\mathrm{~s}, 1 \mathrm{H}), 7.69(\mathrm{~d}$, $J=8.0 \mathrm{~Hz}, 2 \mathrm{H}), 7.21(\mathrm{dd}, J=6.0,2.5 \mathrm{~Hz}, 3 \mathrm{H}), 7.14-7.12(\mathrm{~m}, 3 \mathrm{H}), 7.08(\mathrm{t}, J=7.5 \mathrm{~Hz}, 2 \mathrm{H}), 6.90(\mathrm{~d}, J=$ $8.0 \mathrm{~Hz}, 2 \mathrm{H}), 6.83(\mathrm{~d}, J=7.5 \mathrm{~Hz}, 2 \mathrm{H}), 6.39(\mathrm{~s}, 1 \mathrm{H}), 6.20(\mathrm{~s}, 1 \mathrm{H}), 2.31$ (t, $J=7.5 \mathrm{~Hz}, 2 \mathrm{H}), 2.25(\mathrm{~s}, 3 \mathrm{H})$, $1.45-1.39(\mathrm{~m}, 2 \mathrm{H}), 1.23(\mathrm{~s}, 14 \mathrm{H}), 0.88(\mathrm{t}, J=6.5 \mathrm{~Hz}, 3 \mathrm{H}) .{ }^{13} \mathrm{C}$ NMR $\left(125 \mathrm{MHz}, \mathrm{CDCl}_{3}\right): \delta=166.53$, 143.54, 137.09, 136.21, 135.94, 135.07, 133.83, 133.65, 130.49, 128.66, 128.22, 128.21, 127.67, 127.31, 126.98, 126.87, 126.34, 34.40, 30.88, 28.56, 28.53, 28.29, 28.11, 27.06, 21.66, 20.59, 13.11. HR-MS (ESI): $\mathrm{m} / \mathrm{z}$ calculated for $\mathrm{C}_{34} \mathrm{H}_{41} \mathrm{NO}_{3} \mathrm{~S}:[\mathrm{M}+\mathrm{H}]^{+}: 544.2880$, found: 544.2880. FTIR $\left(\mathrm{KBr}, \mathrm{cm}^{-1}\right)$ : $3626.13,3444.44,2922.75,1694.37,1384.25,1159.81,1083.89,459.45$.<smiles>CCCCCNC(=O)C(C)=C(C)C(=Cc1ccccc1)c1ccccc1</smiles>

(2Z,4Z)-2,3-Mimethyl-4,5-diphenyl- $N$-tosylpenta-2,4-dienamide $\quad$ (3ka).

Following the general experiment procedure, 3ka was obtained as a yellow solid $(30 \%, 25 \mathrm{mg})$. M. p. $=169^{\circ} \mathrm{C} .{ }^{1} \mathrm{H}$ NMR $\left(500 \mathrm{MHz}, \mathrm{CDCl}_{3}\right): \delta=7.65$ (d, $J=8.5 \mathrm{~Hz}, 2 \mathrm{H}), 7.26$ (dd, $J=4.0,2.5 \mathrm{~Hz}, 3 \mathrm{H}), 7.15-7.09$ (m, 5H), 6.85 (d, $J=8.5 \mathrm{~Hz}, 4 \mathrm{H}), 6.30$ (s, 1H), 2.20 (s, 3H), 1.98 (d, $J=1.0 \mathrm{~Hz}, 3 \mathrm{H}), 1.67$ (d, $J=1.0 \mathrm{~Hz}, 3 \mathrm{H}) .{ }^{13} \mathrm{C} \mathrm{NMR}\left(125 \mathrm{MHz}, \mathrm{CDCl}_{3}\right): \delta=$ $166.89,143.95,143.39,141.56,136.19,134.91,134.09,128.63,128.53,128.49,128.07,127.80$, $127.51,127.18,127.05,126.89,126.25,20.57,17.95,15.39$. HR-MS (ESI): $\mathrm{m} / \mathrm{z}$ calculated for $\mathrm{C}_{26} \mathrm{H}_{25} \mathrm{NO}_{3} \mathrm{~S}:[\mathrm{M}+\mathrm{H}]^{+}: 432.1628$, found: 432.1626. FTIR $\left(\mathrm{KBr}, \mathrm{cm}^{-1}\right): 3882.07,3500.66,3363.34$, 2358.43, 1651.52, 1557.50, 1384.19 .

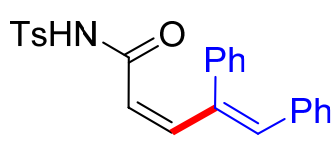

(2Z, 4Z)-4, 5-Diphenyl- $N$-tosylpenta-2,4-dienamide (3la). 
Following the general experiment procedure, 3la was obtained as a yellow solid (40\%, $32 \mathrm{mg})$. M. p. $=216^{\circ} \mathrm{C} .{ }^{1} \mathrm{H}$ NMR $\left(500 \mathrm{MHz}, \mathrm{CDCl}_{3}\right): \delta=8.35(\mathrm{~s}, 1 \mathrm{H}), 7.56(\mathrm{~d}, J=8.0 \mathrm{~Hz}, 2 \mathrm{H}), 7.41-7.32(\mathrm{~m}, 7 \mathrm{H})$, 7.29-7.26 (m, 3H), 7.17 (d, $J=8.5 \mathrm{~Hz}, 2 \mathrm{H}), 7.04$ (dd, $J=9.5,2.0 \mathrm{~Hz}, 2 \mathrm{H}), 6.08(\mathrm{~d}, J=13.0 \mathrm{~Hz}, 1 \mathrm{H})$, $2.40(\mathrm{~s}, 3 \mathrm{H}) .{ }^{13} \mathrm{C}$ NMR $\left(125 \mathrm{MHz}, \mathrm{CDCl}_{3}\right): \delta=161.12,143.60,140.36,138.84,134.90,134.82,134.39$, 132.81, 128.74, 128.30, 127.89, 127.56, 127.52, 127.32, 127.26, 125.79, 124.06, 20.63. HR-MS (ESI): $\mathrm{m} / \mathrm{z}$ calculated for $\mathrm{C}_{24} \mathrm{H}_{21} \mathrm{NO}_{3} \mathrm{~S}$ : $[\mathrm{M}+\mathrm{H}]^{+}: 404.1315$, found: 404.1319. FTIR $\left(\mathrm{KBr}, \mathrm{cm}^{-1}\right): 3850.93$, $3450.69,3416.32,2338.40,1651.59,1633.83,1557.17,1384.19$.

PsHN

(E)-2-(1,2-Diphenylvinyl)- $N$-tosylcyclohex-1-ene-1-carboxamide (3ma).

Following the general experiment procedure, 3ma was obtained as a yellow solid $(87 \%, 80 \mathrm{mg})$. M. p. $=168^{\circ} \mathrm{C} .{ }^{1} \mathrm{H}$ NMR $\left(500 \mathrm{MHz}, \mathrm{CDCl}_{3}\right): \delta=8.74(\mathrm{~s}$, $1 \mathrm{H}), 7.67(\mathrm{~d}, J=8.5 \mathrm{~Hz}, 2 \mathrm{H}), 7.25(\mathrm{dd}, J=5.5,2.5 \mathrm{~Hz}, 3 \mathrm{H}), 7.15-7.09(\mathrm{~m}$, $5 \mathrm{H}), 6.87(\mathrm{~d}, J=8.0 \mathrm{~Hz}, 4 \mathrm{H}), 6.34(\mathrm{~s}, 1 \mathrm{H}), 2.42-2.39(\mathrm{~m}, 2 \mathrm{H}), 2.21(\mathrm{~s}, 3 \mathrm{H}), 1.99-1.96(\mathrm{~m}, 2 \mathrm{H})$, 1.64-1.60 (m, 2H), 1.54-1.51 (m, 2H). ${ }^{13} \mathrm{C}$ NMR (125 MHz, $\left.\mathrm{CDCl}_{3}\right): \delta=166.57,145.89,143.40$, $140.98,136.32$, 134.92, 134.16, 129.19, 128.61, 128.58, 128.34, 128.09, 127.79, 127.21, 126.97, 126.92, 126.26, 28.72, 25.82, 20.93, 20.83, 20.56. HR-MS (ESI): $\mathrm{m} / \mathrm{z}$ calculated for $\mathrm{C}_{28} \mathrm{H}_{27} \mathrm{NO}_{3} \mathrm{~S}$ : $[\mathrm{M}+\mathrm{H}]^{+}:$458.1784, found: 458.1784. FTIR $\left(\mathrm{KBr}, \mathrm{cm}^{-1}\right): 3850.08,3444.39,2923.28,1682.54,1538.71$, $1545.50,1384.23,1164.59$.

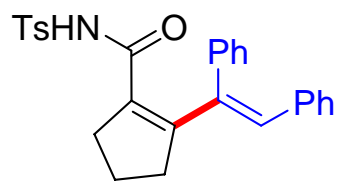

(E)-2-(1,2-Diphenylvinyl)- $N$-tosylcyclopent-1-ene-1-carboxamide (3na). Following the general experiment procedure, 3na was obtained as a yellow solid (72\%, $64 \mathrm{mg})$. M. p. $=225^{\circ} \mathrm{C} .{ }^{1} \mathrm{H}$ NMR $\left(500 \mathrm{MHz}, \mathrm{CDCl}_{3}\right): \delta=7.69$ (d, $J=8.5 \mathrm{~Hz}, 2 \mathrm{H}), 7.30-7.27(\mathrm{~m}, 3 \mathrm{H}), 7.21(\mathrm{dd}, J=5.5,2.5 \mathrm{~Hz}, 3 \mathrm{H}), 7.16(\mathrm{dd}, J=5.5,2.0 \mathrm{~Hz}, 2 \mathrm{H})$, $7.13(\mathrm{~d}, J=8.0 \mathrm{~Hz}, 2 \mathrm{H}), 7.05$ (dd, $J=5.5,2.0 \mathrm{~Hz}, 2 \mathrm{H}), 6.57$ (s, 1H), 2.75 (t, $J=7.5 \mathrm{~Hz}, 2 \mathrm{H}), 2.51$ (t, $J$ $=7.5 \mathrm{~Hz}, 2 \mathrm{H}), 2.37(\mathrm{~s}, 3 \mathrm{H}), 1.86-1.80(\mathrm{~m}, 2 \mathrm{H}) .{ }^{13} \mathrm{C} \mathrm{NMR}\left(125 \mathrm{MHz}, \mathrm{CDCl}_{3}\right): \delta=162.20,153.52$, $143.51,136.77,135.48,134.75,134.27,131.86,130.43,128.53,128.29,128.01,127.95,127.41$, 127.38, 127.31, 127.00, 38.17, 33.34, 20.58, 20.55. HR-MS (ESI): $\mathrm{m} / \mathrm{z}$ calculated for $\mathrm{C}_{27} \mathrm{H}_{25} \mathrm{NO}_{3} \mathrm{~S}$ : $[\mathrm{M}+\mathrm{H}]^{+}:$444.1628, found: 444.1631. FTIR $\left(\mathrm{KBr}, \mathrm{cm}^{-1}\right): 3819.04,3556.93,3406.63,2980.82,1682.37$, $1651.53,1384.22$.

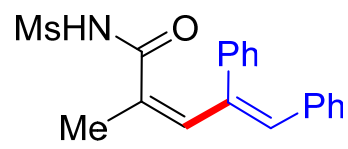

(2Z,4Z)-2-methyl- $N$-(methylsulfonyl)-4,5-diphenylpenta-2,4-dienamide

(3oa). Following the general experiment procedure, 3oa was obtained as a colorless liquid $(87 \%, 59 \mathrm{mg}) .{ }^{1} \mathrm{H}$ NMR $\left(500 \mathrm{MHz}, \mathrm{CDCl}_{3}\right): \delta=$ 7.23-7.22 (m, 3H), 7.16-7.14 (m, 2H), $7.05(\mathrm{dd}, J=5.0,2.0 \mathrm{~Hz}, 3 \mathrm{H}), 6.88(\mathrm{dd}, J=6.0,2.0 \mathrm{~Hz}, 2 \mathrm{H})$, $6.67(\mathrm{~s}, 1 \mathrm{H}), 6.43(\mathrm{t}, J=1.0 \mathrm{~Hz}, 1 \mathrm{H}), 2.77(\mathrm{~s}, 3 \mathrm{H}), 2.01(\mathrm{~d}, J=1.5 \mathrm{~Hz}, 3 \mathrm{H}) .{ }^{13} \mathrm{C}$ NMR $(125 \mathrm{MHz}$, $\left.\mathrm{CDCl}_{3}\right): \delta=168.29,137.66,137.21,136.70,135.89,133.53,131.85,129.67,129.44,128.90,128.23$, 128.21, 127.75, 41.17, 21.45. HR-MS (ESI): $\mathrm{m} / \mathrm{z}$ calculated for $\mathrm{C}_{19} \mathrm{H}_{19} \mathrm{NO}_{3} \mathrm{~S}:[\mathrm{M}+\mathrm{H}]^{+}: 342.1158$, found: 342.1157. FTIR $\left(\mathrm{KBr}, \mathrm{cm}^{-1}\right): 3851.09,3673.66,3520.95,3444.80,2980.82,2340.50,1651.72$, 1384.28. 
(S,E)-2-(1,2-Diphenylvinyl)-4-(prop-1-en-2-yl)- $N$-tosylcyclohex-1-ene-<smiles>C=C(C)[C@H]1CCC(C(=O)Nc2ccccc2)=C(/C(=C\c2ccccc2)c2ccccc2)C1</smiles>

1-carboxamide (3pa). Following the general experiment procedure, 3pa was obtained as a yellow solid $(96 \%, 95 \mathrm{mg})$. M. p. $=145^{\circ} \mathrm{C} .{ }^{1} \mathrm{H}$ NMR $\left(500 \mathrm{MHz}, \mathrm{CDCl}_{3}\right): \delta=8.52(\mathrm{~s}, 1 \mathrm{H}), 7.64(\mathrm{~d}, J=8.5 \mathrm{~Hz}, 2 \mathrm{H}), 7.28-7.29$ (m, 3H), 7.13-7.18 (m, 5H), 6.90-6.93 (m, 4H), $6.36(\mathrm{~s}, 1 \mathrm{H}), 4.68(\mathrm{~s}, 1 \mathrm{H})$, $4.60(\mathrm{~s}, 1 \mathrm{H}), 2.59-2.63(\mathrm{~m}, 1 \mathrm{H}), 2.37-2.43(\mathrm{~m}, 1 \mathrm{H}), 2.24(\mathrm{~s}, 3 \mathrm{H}), 2.08-2.14$ $(\mathrm{m}, 2 \mathrm{H}), 1.93-1.98(\mathrm{~m}, 1 \mathrm{H}), 1.86-1.89(\mathrm{~m}, 1 \mathrm{H}), 1.63(\mathrm{~s}, 3 \mathrm{H}), 1.43-1.46(\mathrm{~m}$, 1H). ${ }^{13} \mathrm{C}$ NMR (125 MHz, $\mathrm{CDCl}_{3}$ ): $\delta=165.87,147.01,146.00,143.47,140.42,135.82,134.66,134.08$, $129.35,128.76,128.59,128.23,128.10,127.99,127.37,127.27,127.07,126.55,108.59,39.39,34.19$, 26.12, 25.76, 20.58, 19.63. HR-MS (ESI): $\mathrm{m} / \mathrm{z}$ calculated for $\mathrm{C}_{31} \mathrm{H}_{31} \mathrm{NO}_{3} \mathrm{~S}:[\mathrm{M}+\mathrm{H}]^{+}: 498.2097$, found: 498.2103. FTIR (KBr, $\left.\mathrm{cm}^{-1}\right): 3850.91,3742.22$, 3646.11, 3626.54, 2358.43, 2340.31, 1651.53, 1402.76, 1385.09 .<smiles>C=C(C)[C@H]1CCC(C(=O)N[Sb])=C(/C(=C\CCCCC)CCCCC)C1</smiles>

(S,E)-2-(Dodec-6-en-6-yl)-4-(prop-1-en-2-yl)- $N$-tosylcyclohex-1ene-1-carboxamide (3pg).

Following the general experiment procedure, 3pg was obtained as a yellow liquid $(71 \%, 69 \mathrm{mg}) .{ }^{1} \mathrm{H} \mathrm{NMR}\left(500 \mathrm{MHz}, \mathrm{CDCl}_{3}\right): \delta=$ $8.81(\mathrm{~s}, 1 \mathrm{H}), 7.93-7.95(\mathrm{~m}, 2 \mathrm{H}), 7.31(\mathrm{~d}, J=8.0 \mathrm{~Hz}, 2 \mathrm{H}), 5.40$ (t, $J$ $=7.5 \mathrm{~Hz}, 1 \mathrm{H}), 4.75(\mathrm{t}, J=1.5 \mathrm{~Hz}, 1 \mathrm{H}), 4.69(\mathrm{~s}, 1 \mathrm{H}), 2.47-2.51(\mathrm{~m}, 1 \mathrm{H}), 2.43(\mathrm{~s}, 3 \mathrm{H}), 2.21-2.27(\mathrm{~m}, 1 \mathrm{H})$, 2.05-2.12 (m, 5H), 1.96-2.02 (m, 1H), 1.81-1.85 (m, 1H), $1.72(\mathrm{~s}, 3 \mathrm{H}), 1.20-1.45(\mathrm{~m}, 14 \mathrm{H}), 0.92(\mathrm{t}, J=$ $7.0 \mathrm{~Hz}, 3 \mathrm{H}), 0.87$ (t, $J=7.0 \mathrm{~Hz}, 3 \mathrm{H}) .{ }^{13} \mathrm{C}$ NMR $\left(125 \mathrm{MHz}, \mathrm{CDCl}_{3}\right): \delta=165.14,148.13,147.31,143.71$, $140.91,134.89,130.47,128.35,127.53,125.74,108.43,39.69,37.49,30.99,30.69,30.38,27.92,27.21$, 26.96, 25.80, 25.16, 21.50, 21.27, 20.62, 19.67, 13.01, 12.97. HR-MS (ESI): $\mathrm{m} / \mathrm{z}$ calculated for $\mathrm{C}_{29} \mathrm{H}_{43} \mathrm{NO}_{3} \mathrm{~S}:[\mathrm{M}+\mathrm{H}]^{+}: 486.3036$, found: 486.3041. FTIR $\left(\mathrm{KBr}, \mathrm{cm}^{-1}\right): 3626.50,3564.48,3175.23$, 2358.03, 1651.51, 1557.22, 1455.26, 1402.64, 1385.10.

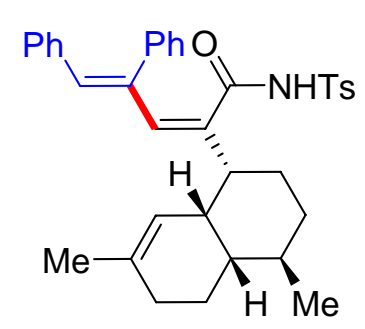

(2E,4Z)-2-((1R,4R,4aS,8aR)-4,7-Dimethyl-1,2,3,4,4a,5,6,8a-octahydrona phthalen-1-yl)-4,5-diphenyl- $N$-tosylpenta-2,4-dienamide (3qa).

Following the general experiment procedure, 3qa was obtained as a yellow liquid $(86 \%, 97 \mathrm{mg}) .{ }^{1} \mathrm{H}$ NMR $\left(500 \mathrm{MHz}, \mathrm{CDCl}_{3}\right): \delta=8.43(\mathrm{~s}, 1 \mathrm{H}), 7.67$ (d, $J=8.5 \mathrm{~Hz}, 2 \mathrm{H}), 7.24-7.26(\mathrm{~m}, 3 \mathrm{H}), 7.12-7.18(\mathrm{~m}, 3 \mathrm{H}), 7.07(\mathrm{t}, J=7.5 \mathrm{~Hz}$, $2 \mathrm{H}), 6.81-6.86(\mathrm{dd}, J=8.5 \mathrm{~Hz}, J=19.0 \mathrm{~Hz}, 4 \mathrm{H}), 6.38(\mathrm{~s}, 1 \mathrm{H}), 6.05(\mathrm{~s}, 1 \mathrm{H})$, $5.10(\mathrm{~s}, 1 \mathrm{H}), 2.66(\mathrm{~d}, J=12.5 \mathrm{~Hz}, 1 \mathrm{H}), 2.38(\mathrm{~s}, 1 \mathrm{H}), 2.24(\mathrm{~s}, 3 \mathrm{H}), 1.82-1.89(\mathrm{~m}, 2 \mathrm{H}), 1.71-1.75(\mathrm{~m}, 1 \mathrm{H})$, 1.64-1.67 (m, 1H), $1.59(\mathrm{~s}, 3 \mathrm{H}), 1.41-1.47(\mathrm{~m}, 2 \mathrm{H}), 1.33-1.37(\mathrm{~m}, 1 \mathrm{H}), 1.24-1.31(\mathrm{~m}, 2 \mathrm{H}), 0.98-1.03(\mathrm{~m}$, $1 \mathrm{H}), 0.85(\mathrm{~d}, J=6.0 \mathrm{~Hz}, 3 \mathrm{H}) .{ }^{13} \mathrm{C}$ NMR $\left(125 \mathrm{MHz}, \mathrm{CDCl}_{3}\right): \delta=167.09,143.49,139.14,137.35$, $136.08,135.13$, 134.86, 133.67, 132.61, 130.21, 128.67, 128.31, 128.08, 127.72, 127.37, 127.02, 126.84, 126.27, 118.44, 44.47, 40.21, 36.84, 34.00, 26.43, 25.32, 24.50, 24.19, 22.90, 20.59, 18.61 . HR-MS (ESI): $\mathrm{m} / \mathrm{z}$ calculated for $\mathrm{C}_{36} \mathrm{H}_{39} \mathrm{NO}_{3} \mathrm{~S}:[\mathrm{M}+\mathrm{H}]^{+}: 566.2723$, found: 566.2715 . FTIR $\left(\mathrm{KBr}, \mathrm{cm}^{-1}\right)$ : 3850.91, 3646.03, 3224.94, 1682.37, 1667.42, 1422.72, 1403.10, 1345.58, 1156.85, 1120.77, 1087.78. 


\section{Gram-Scale Synthesis of 3aa}<smiles>C=C(C)C(=O)NCCCCCC</smiles>

$1 \mathrm{a}, 1.0 \mathrm{~g}$<smiles>[PH3+]#CPc1ccccc1</smiles>

$2 a$

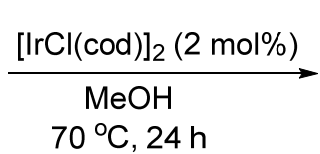

$70^{\circ} \mathrm{C}, 24 \mathrm{~h}$<smiles>C/C(=C/C(=C/c1ccccc1)c1ccccc1)C(=O)N[TeH]</smiles>

3aa, $80 \%$

An oven-dried vial was charged with $[\operatorname{Ir}(\operatorname{cod}) \mathrm{Cl}]_{2}(56.2 \mathrm{mg}, 2 \mathrm{~mol} \%), \mathrm{MeOH}(21 \mathrm{~mL})$. Then, alkyne 2a (1.12 g, $6.27 \mathrm{mmol})$ and acrylamide $1 \mathrm{a}(1.00 \mathrm{~g}, 4.18 \mathrm{mmol})$ were added into the solution in sequence. The vial was sealed under argon and heated to $70{ }^{\circ} \mathrm{C}$ with stirring for 24 hours. After cooling down, the mixture was directly applied to a flash column chromatography (EtOAc/petroleum ether mixtures). The desires product 3aa was obtained as a yellow solid (1.40 g, 80\%).

\section{Deuterium-Labeled Experiments}

\section{H/D Exchange in 1b}

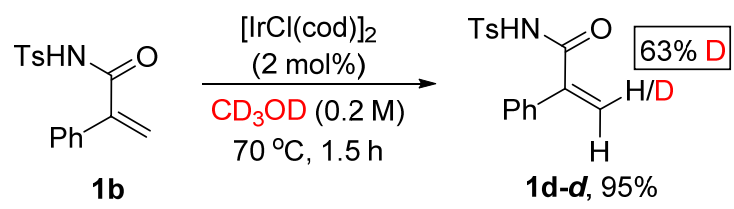

An oven-dried vial was charged with $[\operatorname{Ir}(\operatorname{cod}) \mathrm{Cl}]_{2}(1.3 \mathrm{mg}, 2 \mathrm{~mol} \%)$, acrylamide $\mathbf{1 b}(60.3 \mathrm{mg}, 0.2$ mmol) and $\mathrm{CD}_{3} \mathrm{OD}(1 \mathrm{~mL})$. The vial was sealed under argon and heated to $70{ }^{\circ} \mathrm{C}$ with stirring for 1.5 hours. After cooling down, the mixture was directly applied to a flash column chromatography (EtOAc/petroleum ether mixtures) on silica gel to afford acrylamide 1d-d (57.3 $\mathrm{mg}$, 95\% recovered). The D \% of $\mathbf{1 b}-\boldsymbol{d}$ was estimated by ${ }^{1} \mathrm{H}$ NMR.
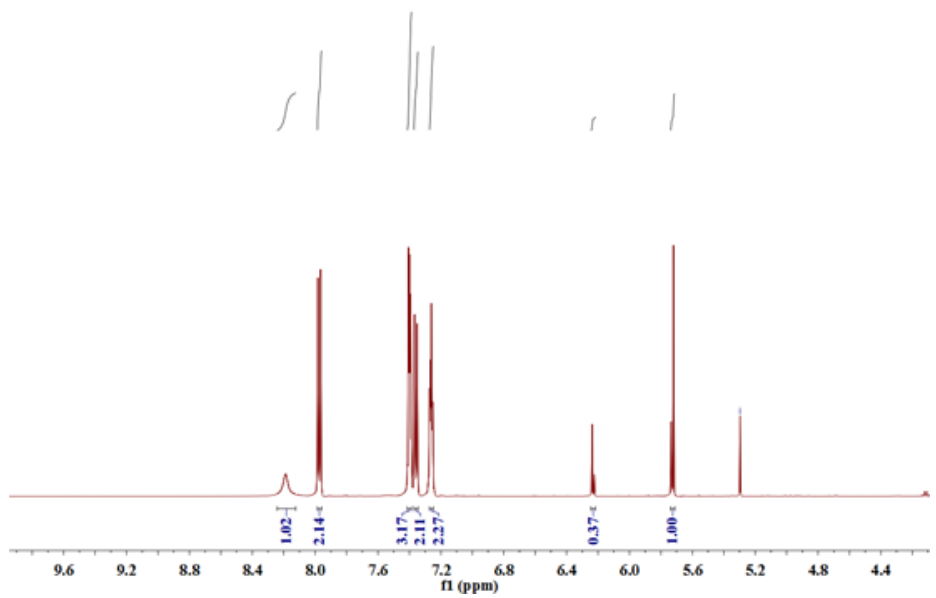


\section{Selective Deuterium-Labeled Synthesis}

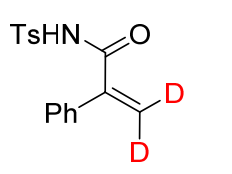

$1 \mathrm{~b}-\mathrm{d}_{2}$<smiles>C(#Cc1ccccc1)c1ccccc1</smiles>

$2 a$

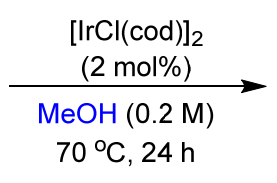

$70^{\circ} \mathrm{C}, 24 \mathrm{~h}$

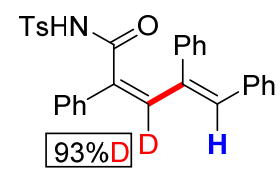

3ba-d, $93 \%$

A $10 \mathrm{~mL}$ vial was charged with $[\operatorname{Ir}(\operatorname{cod}) \mathrm{Cl}]_{2}(1.4 \mathrm{mg}, 2 \mathrm{~mol} \%), \mathrm{MeOH}(1 \mathrm{~mL})$. Then, alkyne 2a (26.8 $\mathrm{mg}, 0.3 \mathrm{mmol})$ and acrylamide $\mathbf{1 b}-\boldsymbol{d}_{2}(30.4 \mathrm{mg}, 0.1 \mathrm{mmol})$ were added into the solution in sequence. The vial was sealed under $\mathrm{Ar}$ and heated to $70{ }^{\circ} \mathrm{C}$ with stirring for $24 \mathrm{~h}$. After cooling down, the mixture was concentrated in vacuo and purified by column chromatography, affording the product 3ba-d as a yellow solid (44.9 mg, 93\%).
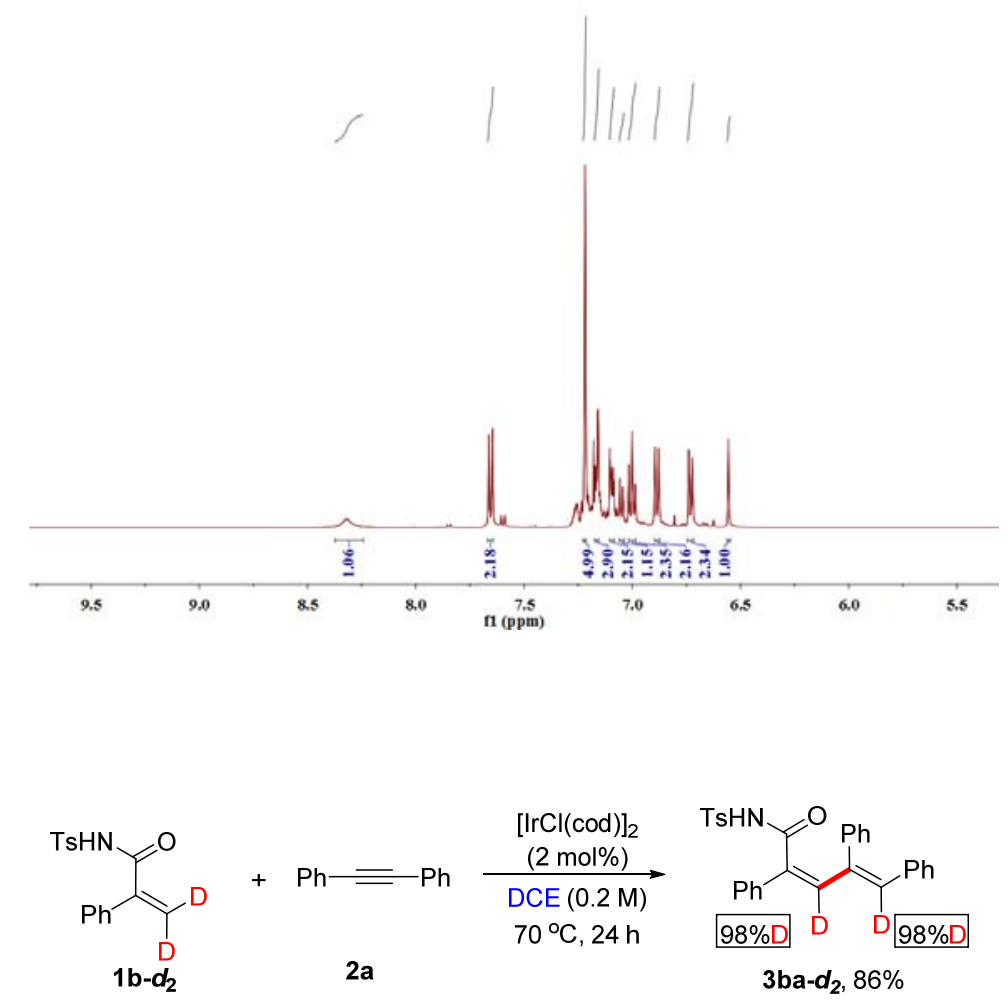

A $10 \mathrm{~mL}$ vial was charged with $[\operatorname{Ir}(\operatorname{cod}) \mathrm{Cl}]_{2}(1.4 \mathrm{mg}, 2 \mathrm{~mol} \%)$, DCE $(1 \mathrm{~mL})$. Then, alkyne $2 a(26.8$ $\mathrm{mg}, 0.15 \mathrm{mmol})$ and acrylamide $\mathbf{1 b}-\boldsymbol{d}_{2}(30.4 \mathrm{mg}, 0.1 \mathrm{mmol})$ were added into the solution in sequence. The vial was sealed under $\mathrm{Ar}$ and heated to $70{ }^{\circ} \mathrm{C}$ with stirring for 24 hours. After cooling down, the mixture was concentrated in vacuo and purified by column chromatography, affording the product 3ba- $\boldsymbol{d}_{2}$ as a yellow solid ( $\left.41.4 \mathrm{mg}, 86 \%\right)$. 

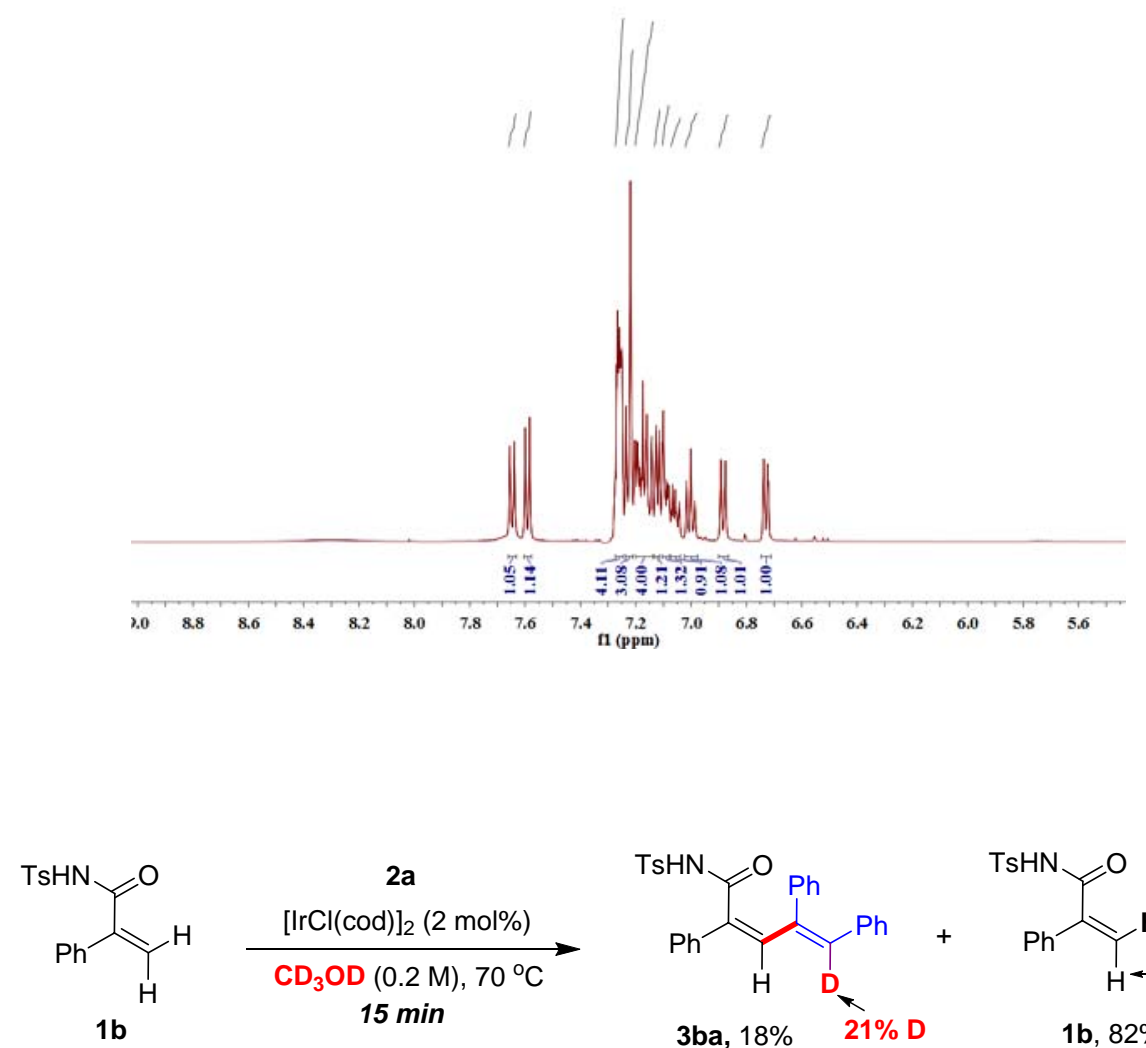

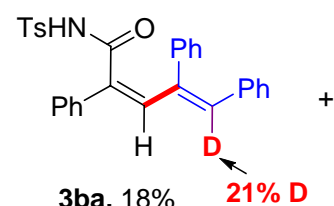

3ba, 18\%

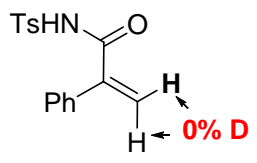

1b, $82 \%$

(recovered)

A $10 \mathrm{~mL}$ vial was charged with $[\operatorname{Ir}(\operatorname{cod}) \mathrm{Cl}]_{2}(2.8 \mathrm{mg}, 2 \mathrm{~mol} \%)$, alkyne 2 a $(107.0 \mathrm{mg}, 0.3 \mathrm{mmol})$, $\mathrm{MeOH}(1.0 \mathrm{~mL})$. Then, acrylamide $1 \mathbf{b}(60.4 \mathrm{mg}, 0.2 \mathrm{mmol})$ awere added into the solution in sequence. The vial was sealed under Ar and heated to $70{ }^{\circ} \mathrm{C}$ with stirring for $15 \mathrm{~min}$. After cooling down, the mixture was directly applied to column chromatography for separation. The $\mathrm{D} \%$ incorporation of product and recovered starting material were determined by ${ }^{1} \mathrm{H}$ NMR.

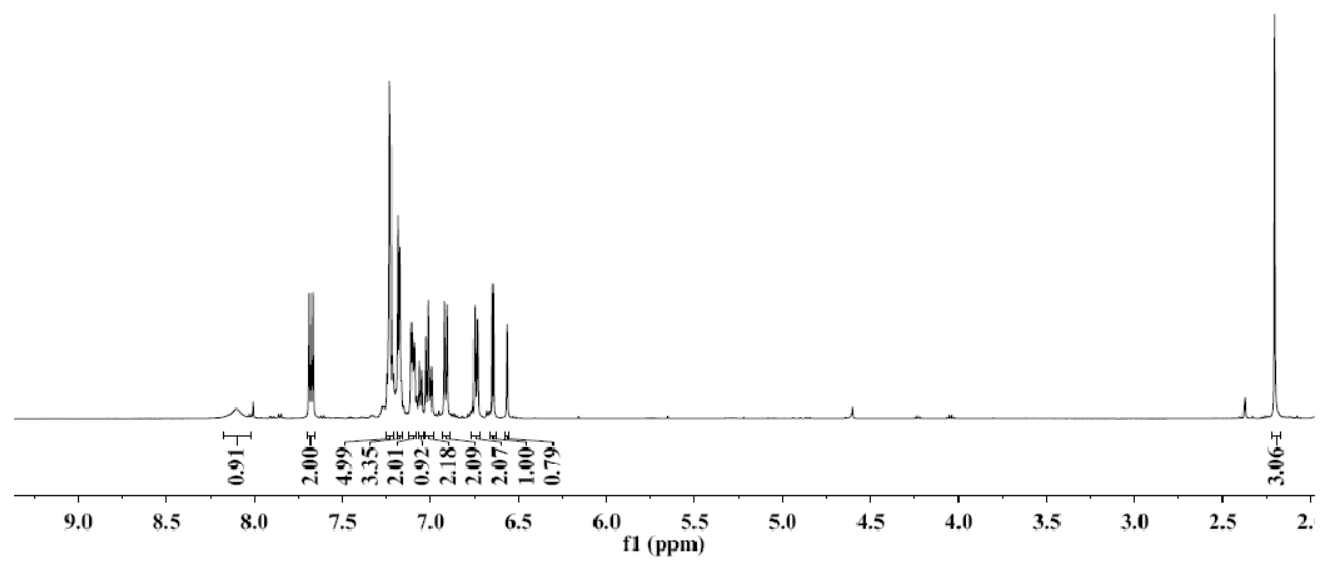




\section{Competitive KIE Experiments with $1 \mathrm{~b}$ and $1 \mathrm{~b}-\mathrm{d}_{2}$}

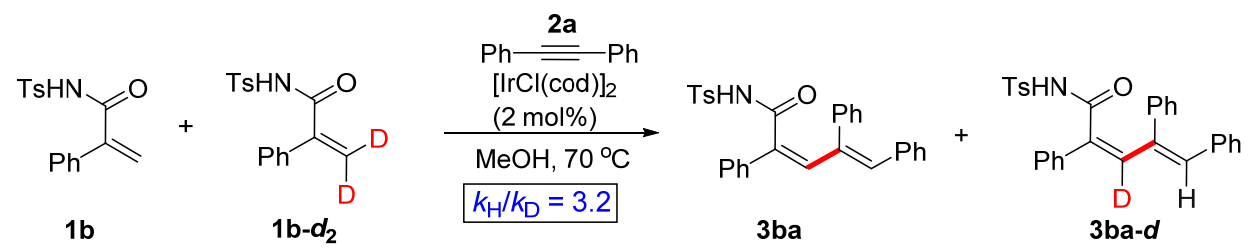

A $10 \mathrm{~mL}$ vial was charged with $[\operatorname{Ir}(\operatorname{cod}) \mathrm{Cl}]_{2}(1.4 \mathrm{mg}, 2 \mathrm{~mol} \%)$, alkyne $2 \mathrm{a}(53.5 \mathrm{mg}, 0.15 \mathrm{mmol})$, MeOH (1.0 mL). Then, acrylamide $\mathbf{1 b}(30.2 \mathrm{mg}, 0.1 \mathrm{mmol})$ and $\mathbf{1 b}-\boldsymbol{d}_{2}(30.4 \mathrm{mg}, 0.1 \mathrm{mmol})$ were added into the solution in sequence. The vial was sealed under $\mathrm{Ar}$ and heated to $70{ }^{\circ} \mathrm{C}$ with stirring for 0.5 hours. After cooling down, the mixture was directly applied to column chromatography for separation. The ratio of $3 \mathbf{b a} / \mathbf{3 b a}-\mathbf{d}$ ( $22.5 \mathrm{mg}, 23 \%$ yield) was determined to be 3.2 by ${ }^{1} \mathrm{H}$ NMR.

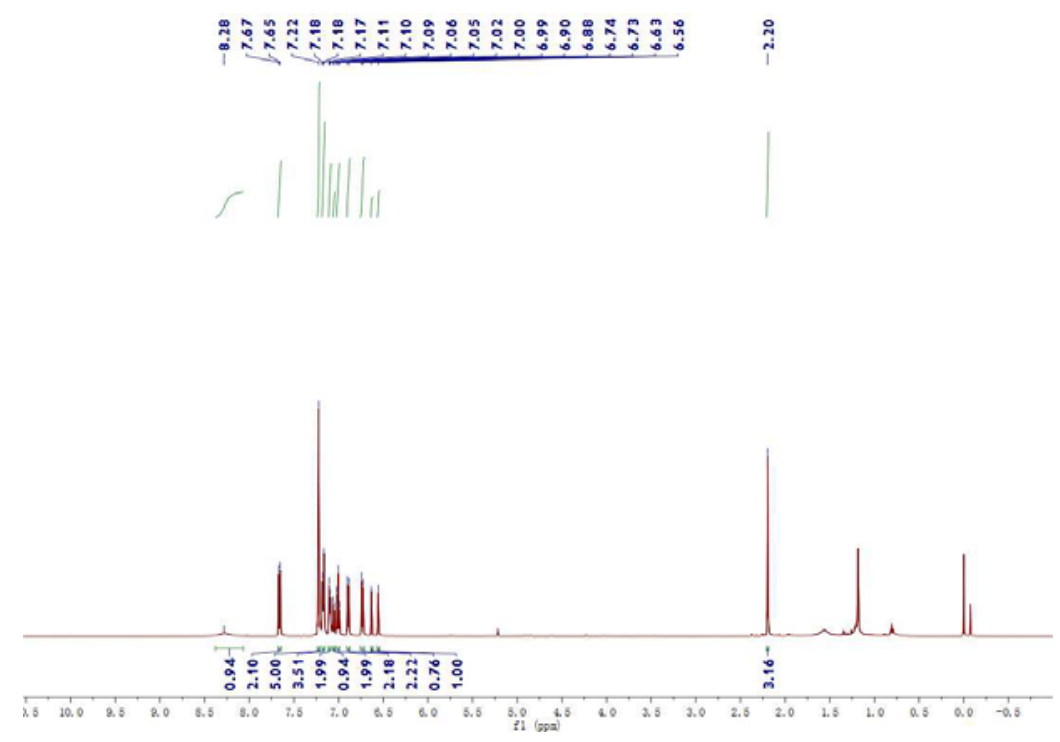

\section{Parallel KIE Experiments with $1 \mathrm{~b}$ and $1 \mathrm{~b}-\mathrm{d}_{2}$}

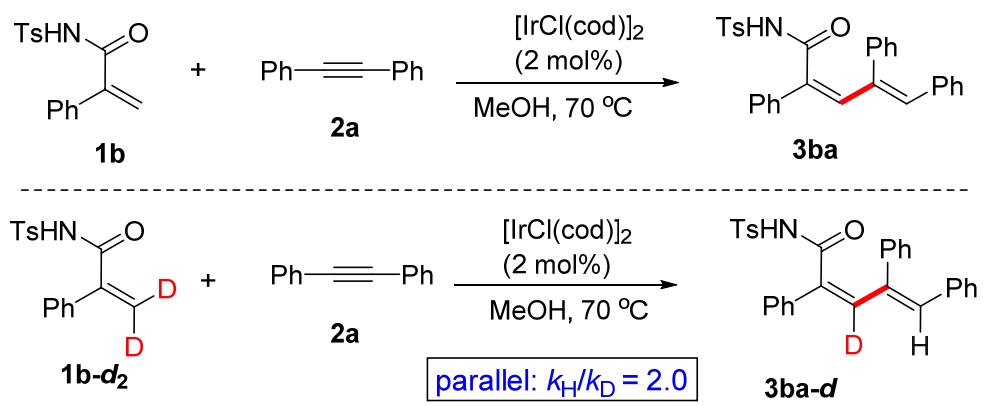

Parallel independent reactions of $\mathbf{1 b} / \mathbf{1} \mathbf{b}-\mathbf{d}_{\mathbf{2}}$ with $\mathbf{2 a}$ were performed to determine the corresponding KIE value. Each $10 \mathrm{~mL}$ vials was charged with $[\operatorname{Ir}(\operatorname{cod}) \mathrm{Cl}]_{2}(1.4 \mathrm{mg}, 2.0 \mathrm{~mol} \%), \mathrm{MeOH}(1 \mathrm{~mL})$. Then, alkyne $\mathbf{2 a}(26.8 \mathrm{mg}, 0.15 \mathrm{mmol})$ and acrylamide $\mathbf{1 b}(30.2 \mathrm{mg}, 0.1 \mathrm{mmol})$ or $\mathbf{1 b}-\boldsymbol{d}_{2}(30.4 \mathrm{mg}, 0.1 \mathrm{mmol})$ were added into the solution in sequence. The vial was sealed under $\mathrm{Ar}$ and heated to $70{ }^{\circ} \mathrm{C}$ with stirring for 5, 10, 15 or 20 minutes. After cooling down, the mixture was concentrated in vacuo and 
purified by column chromatography, affording the product 3ba or 3ba- $\boldsymbol{d}_{2}$. The KIE value was determined to be 2.0 .

\begin{tabular}{c|c|c|c|c}
\hline 3ba & $6.1 \%$ & $11.3 \%$ & $15.1 \%$ & $19.5 \%$ \\
\hline 3ba-d _ $_{2}$ & $4.8 \%$ & $7.3 \%$ & $9.7 \%$ & $11.2 \%$ \\
\hline
\end{tabular}

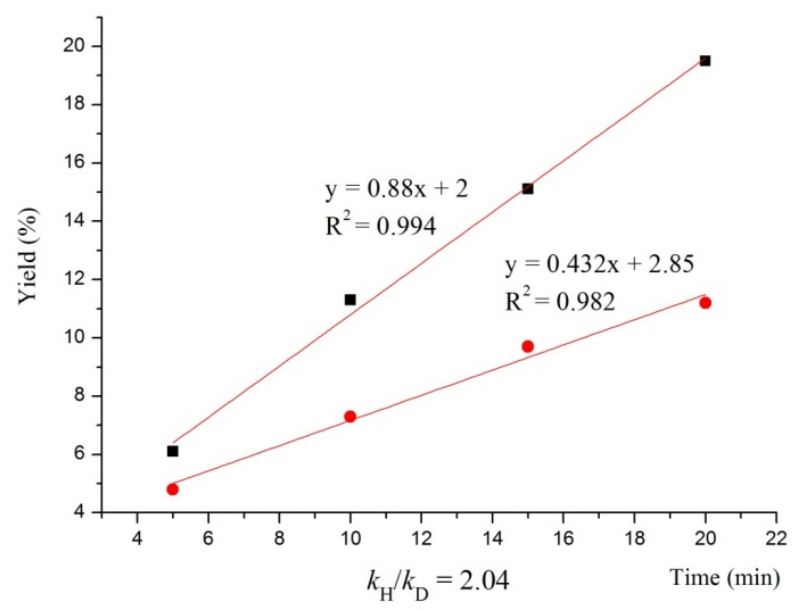

\section{Competitive Reaction of Different Alkynes 2}<smiles>C=C([NH2+]C#CPc1ccccc1)C(=O)c1ccccc1</smiles>

1b 2a

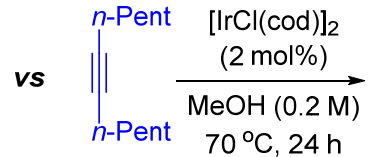

2g<smiles>CNC(=S)NC(=O)C(=CC(=Cc1ccccc1)c1ccccc1)c1ccccc1</smiles>

3ba, $25 \%$<smiles>CCCCC/C=C(\C=C(\C(=O)NCCCC)c1ccccc1)CCCCC</smiles>

3bg, $74 \%$

A $10 \mathrm{~mL}$ vial was charged with $[\operatorname{Ir}(\operatorname{cod}) \mathrm{Cl}]_{2}(2.7 \mathrm{mg}, 2 \mathrm{~mol} \%), \mathrm{MeOH}(1 \mathrm{~mL})$. Then, diphenylacetylene $\mathbf{2 a}(35.6 \mathrm{mg}, 0.2 \mathrm{mmol})$, dodec-6-yne $\mathbf{2 g}(33.3 \mathrm{mg}, 0.2 \mathrm{mmol})$ and acrylamide $\mathbf{1 b}$ (60.3mg, $0.2 \mathrm{mmol}$ ) were added into the solution in sequence. The vial was sealed under Ar and heated to $70{ }^{\circ} \mathrm{C}$ with stirring for 24 hour. After cooling down, the mixture was separated by column chromatography to afford the product 3 ba $(23.9 \mathrm{mg}, 25 \%)$ and $3 \mathrm{bg}(68.8 \mathrm{mg}, 74 \%)$.

\section{Competitive Reaction of Different Acrylamides 1}<smiles>C=C(C(=O)NC)c1ccc(C(F)(F)F)cc1</smiles>

1d<smiles>C=C(C(=O)NCCCCCC)c1ccc(OC)cc1</smiles>

$1 c$

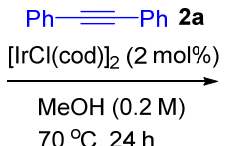

$70^{\circ} \mathrm{C}, 24 \mathrm{~h}$<smiles>O=C(N=[In])C(=CC(=Cc1ccccc1)c1ccccc1)c1ccc(C(F)(F)F)cc1</smiles>

$3 \mathrm{da}, 23 \%$<smiles>CNC(=O)C(=CC(=Cc1ccccc1)c1ccccc1)c1ccc(OC)cc1</smiles>

3ca, $6 \%$ 
A $10 \mathrm{~mL}$ vial was charged with $\left[\operatorname{Ir}(\operatorname{cod}) \mathrm{Cl}_{2}(2.7 \mathrm{mg}, 2 \mathrm{~mol} \%), \mathrm{MeOH}(1 \mathrm{~mL})\right.$. Then, alkyne 2a (53.5 mg, $0.3 \mathrm{mmol})$, acrylamide $1 \mathbf{d}(73.9 \mathrm{mg}, 0.2 \mathrm{mmol})$ and $1 \mathrm{c}(66.2 \mathrm{mg}, 0.2 \mathrm{mmol})$ were added into the solution in sequence. The vial was sealed under Ar and heated to $70{ }^{\circ} \mathrm{C}$ with stirring for 24 hours. After cooling down, the mixture was concentrated in vacuo and purified by column chromatography to afford the product 3da (24.4 mg, 23\%) and 3ca (7.2 mg, 6\%).

\section{Amide Group Removal}<smiles>[3H]NC(=O)/C(C)=C\C(=C\c1ccccc1)c1ccccc1</smiles>

3aa

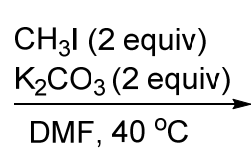

step 1

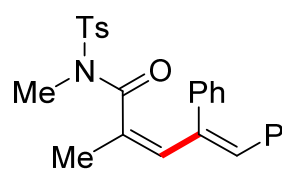

$7,95 \%$

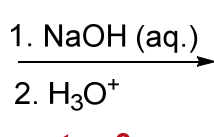

step 2

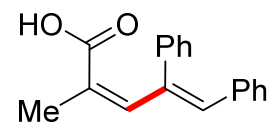

8, $69 \%$

Step 1: $\mathrm{K}_{2} \mathrm{CO}_{3}(27.7 \mathrm{mg}, 0.2 \mathrm{mmol})$ and iodomethane $(28.4 \mathrm{mg}, 0.2 \mathrm{mmol})$ were added to a stirred solution of diene 3aa $(0.1 \mathrm{mmol})$ in DMF $(1.0 \mathrm{ml})$. After stirring at $40{ }^{\circ} \mathrm{C}$ for 3 hours, the solution was evaporated, diluted with water $(10 \mathrm{~mL})$ and the resulting mixture was extracted with ethyl acetate $(3 \mathrm{x}$ $20 \mathrm{~mL}$ ). The combined organic layers were dried over $\mathrm{Na}_{2} \mathrm{SO}_{4}$, filtrated and evaporated. The crude product was purified by flash column chromatography using petroleum ether/ethyl acetate $=30: 1$ as the eluent to give product 7 as a yellow oil $(95 \%, 41 \mathrm{mg}) .{ }^{1} \mathrm{H}$ NMR $\left(500 \mathrm{MHz}, \mathrm{CDCl}_{3}\right): \delta=7.71(\mathrm{~d}, J=8.5$ Hz, 2H), 7.22-7.19 (m, 3H), $7.08-7.03$ (m, 7H), $6.77-6.76$ (m, 2H), 6.45 (s, 1H), 6.17 (s, 1H), 3.11 (s, 3H), $2.31(\mathrm{~s}, 3 \mathrm{H}), 2.05(\mathrm{~d}, J=1.5 \mathrm{~Hz}, 3 \mathrm{H})$.

Step 2: To a solution of compound 7 (41 mg, $0.095 \mathrm{mmol})$ in 1,4-dioxane $(2.5 \mathrm{~mL})$ was added $\mathrm{NaOH}$ $(6 \mathrm{M}, 2.5 \mathrm{~mL})$. The mixture was heated to $60{ }^{\circ} \mathrm{C}$ with stirring for 16 hours. After cooling to room temperature, the solution was concentrated, diluted with water $(10 \mathrm{~mL})$, acidified with $\mathrm{HCl}(3 \mathrm{M})$ and extracted with ethyl acetate $(2 \times 10 \mathrm{~mL})$. The combined organic layers were dried over $\mathrm{Na}_{2} \mathrm{SO}_{4}$, filtrated and evaporated. The crude product was purified by flash column chromatography using petroleum ether/ethyl acetate $=10: 1$ as the eluent to give the product 8 as a colorless oil $(69 \%, 17.3 \mathrm{mg}) .{ }^{1} \mathrm{H}$ NMR $\left(500 \mathrm{MHz}, \mathrm{CDCl}_{3}\right): \delta=7.11-7.08(\mathrm{~m}, 5 \mathrm{H}), 7.02-7.00(\mathrm{~m}, 3 \mathrm{H}), 6.89-6.87(\mathrm{~m}, 2 \mathrm{H}), 6.54(\mathrm{~s}, 1 \mathrm{H}), 6.37(\mathrm{t}$, $J=1.5 \mathrm{~Hz}, 1 \mathrm{H}), 1.94(\mathrm{~d}, J=1.5 \mathrm{~Hz}, 3 \mathrm{H}) .{ }^{13} \mathrm{C} \mathrm{NMR}\left(125 \mathrm{MHz}, \mathrm{CDCl}_{3}\right): \delta=173.63,137.72,137.56$, 137.08, 135.61, 130.31, 128.66, 128.46, 128.18, 127.27, 126.90, 126.46, 126.05, 20.13. HR-MS (ESI): $\mathrm{m} / \mathrm{z}$ calculated for $\mathrm{C}_{18} \mathrm{H}_{16} \mathrm{NO}_{2} \mathrm{~S}:[\mathrm{M}+\mathrm{H}]^{+}:$265.1223, found: 265.1221. FTIR ( $\left.\mathrm{KBr}, \mathrm{cm}^{-1}\right): 3794.03$, $3500.17,3444.85,2922.17,1621.50,1384.30,1258.86,1026.75$.

\section{References}

1. K. Meng, J. Zhang, F. Li, Z. Lin, K. Zhang, G. Zhong, Org. Lett. 2017, 19, 2498.

2. C. Feng, D. Feng, T. P. Loh, Chem. Commun. 2015, 51, 342. 


\section{NMR Spectra}<smiles>CC(=CC(=Cc1ccccc1)c1ccccc1)C(=O)N[TeH]</smiles>

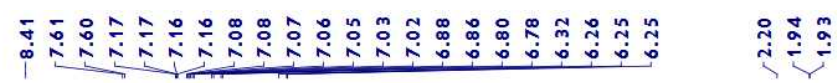

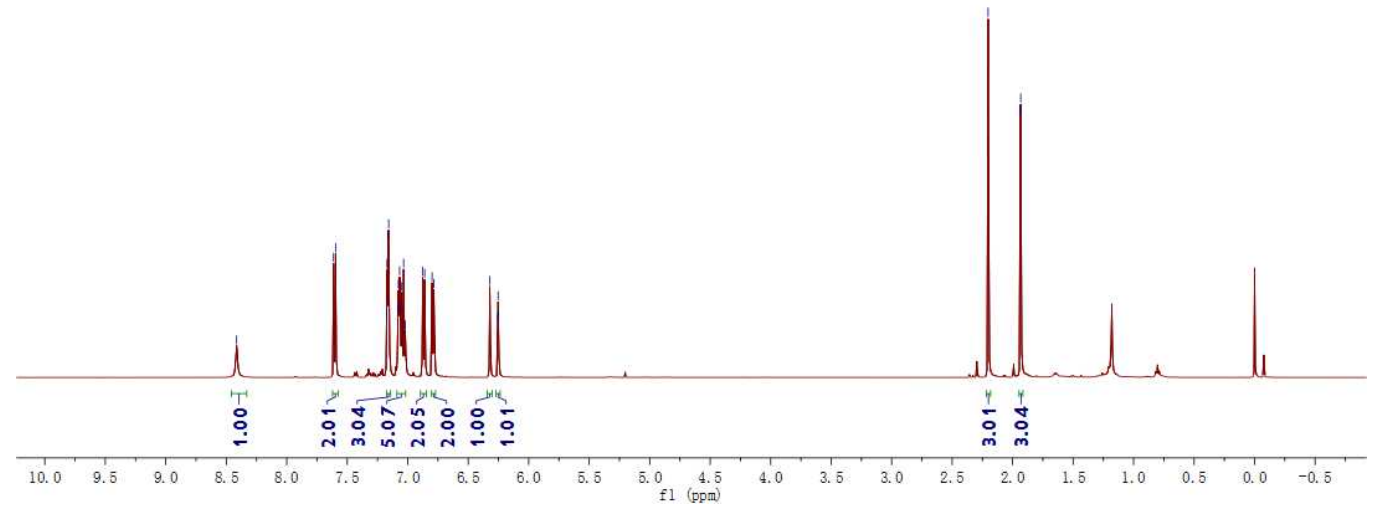

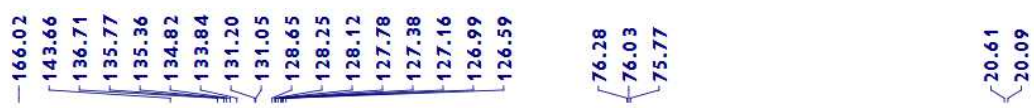

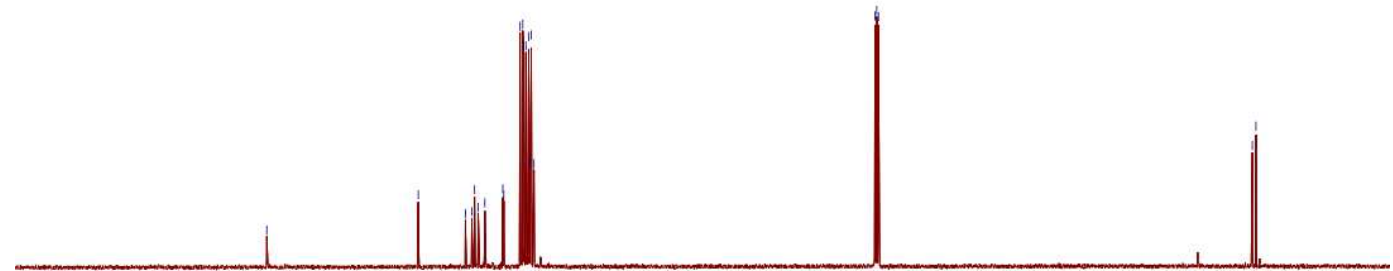

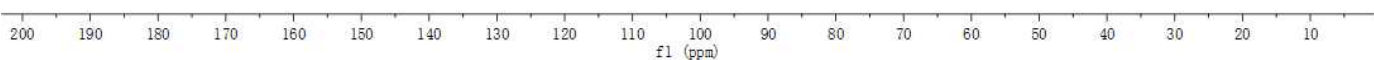


(3ab)

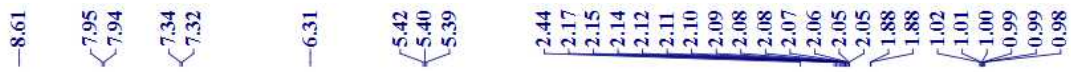
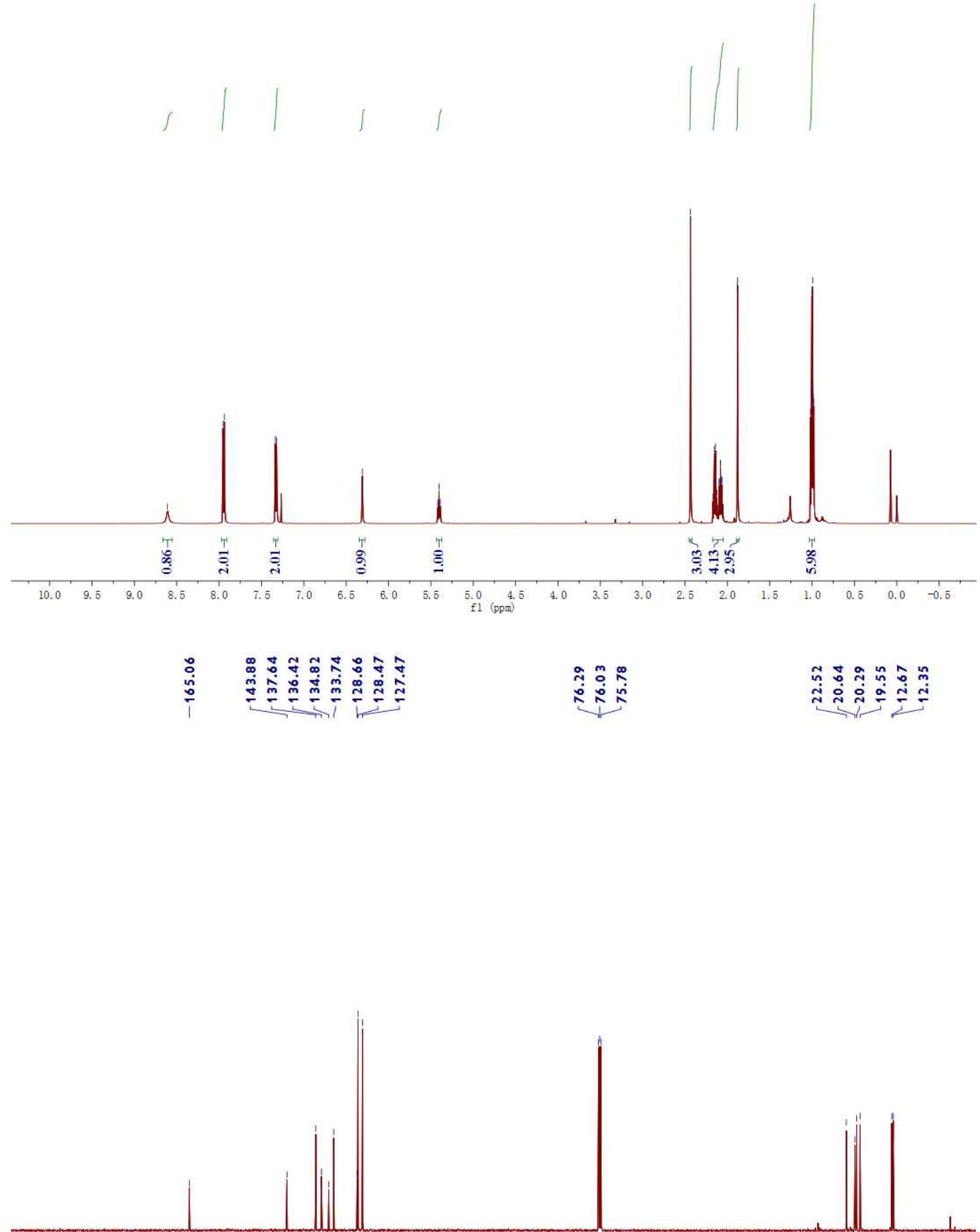

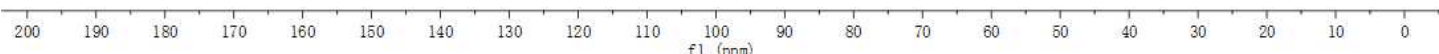



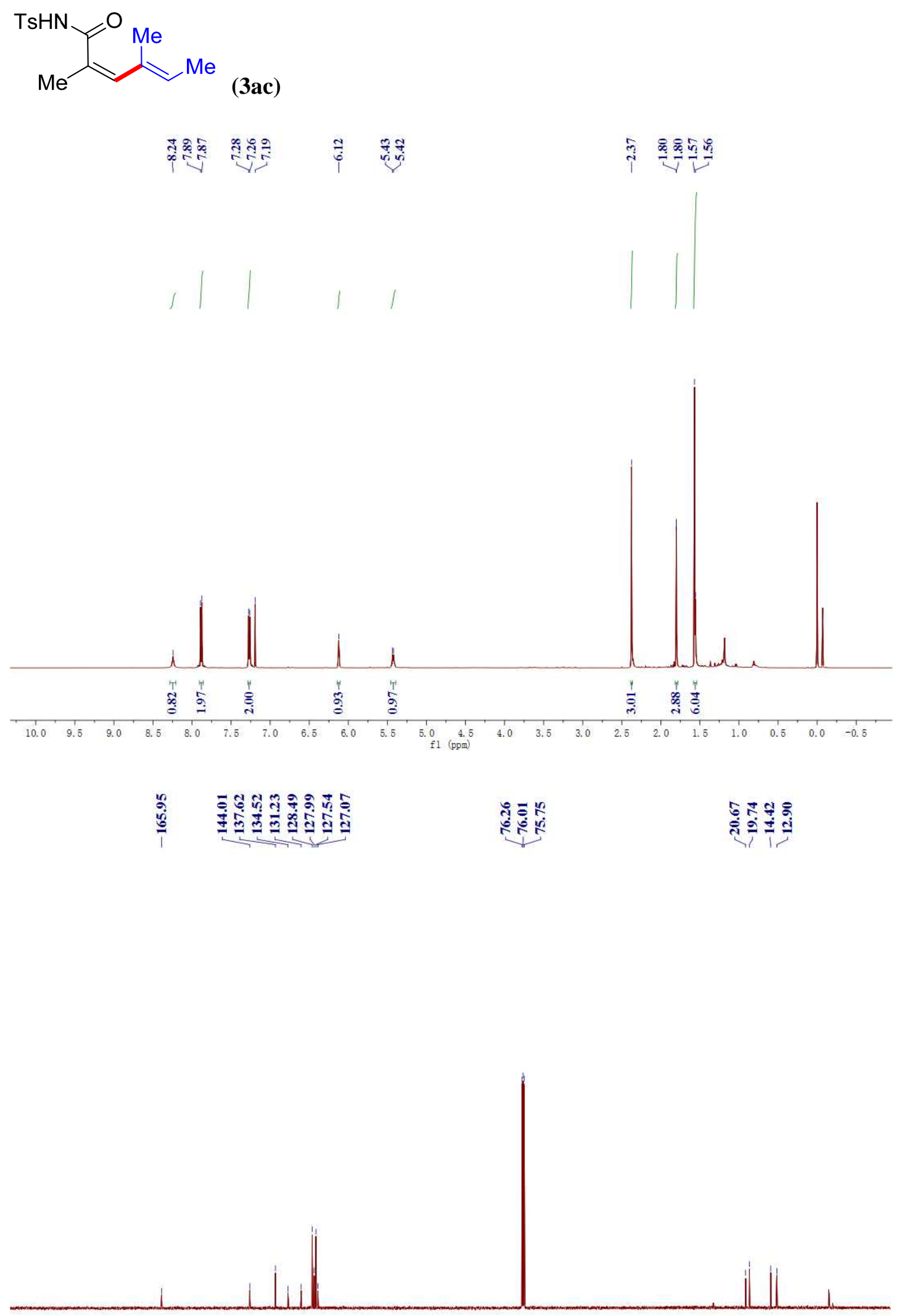

$\begin{array}{lllllllllllllllllllll}200 & 190 & 180 & 170 & 160 & 150 & 140 & 130 & 120 & 110 & \begin{array}{c}100 \\ \mathrm{f} 1(\mathrm{ppm})\end{array} & 80 & 70 & 60 & 50 & 40 & 30 & 20 & 10 & 0 & -10\end{array}$ 
<smiles>C/C(=C/C(=C/c1ccc(CC(C)(C)C)cc1)c1ccc(C(C)(C)C)cc1)C(=O)N[In]</smiles>

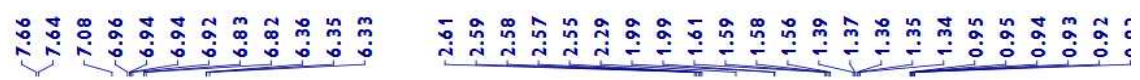
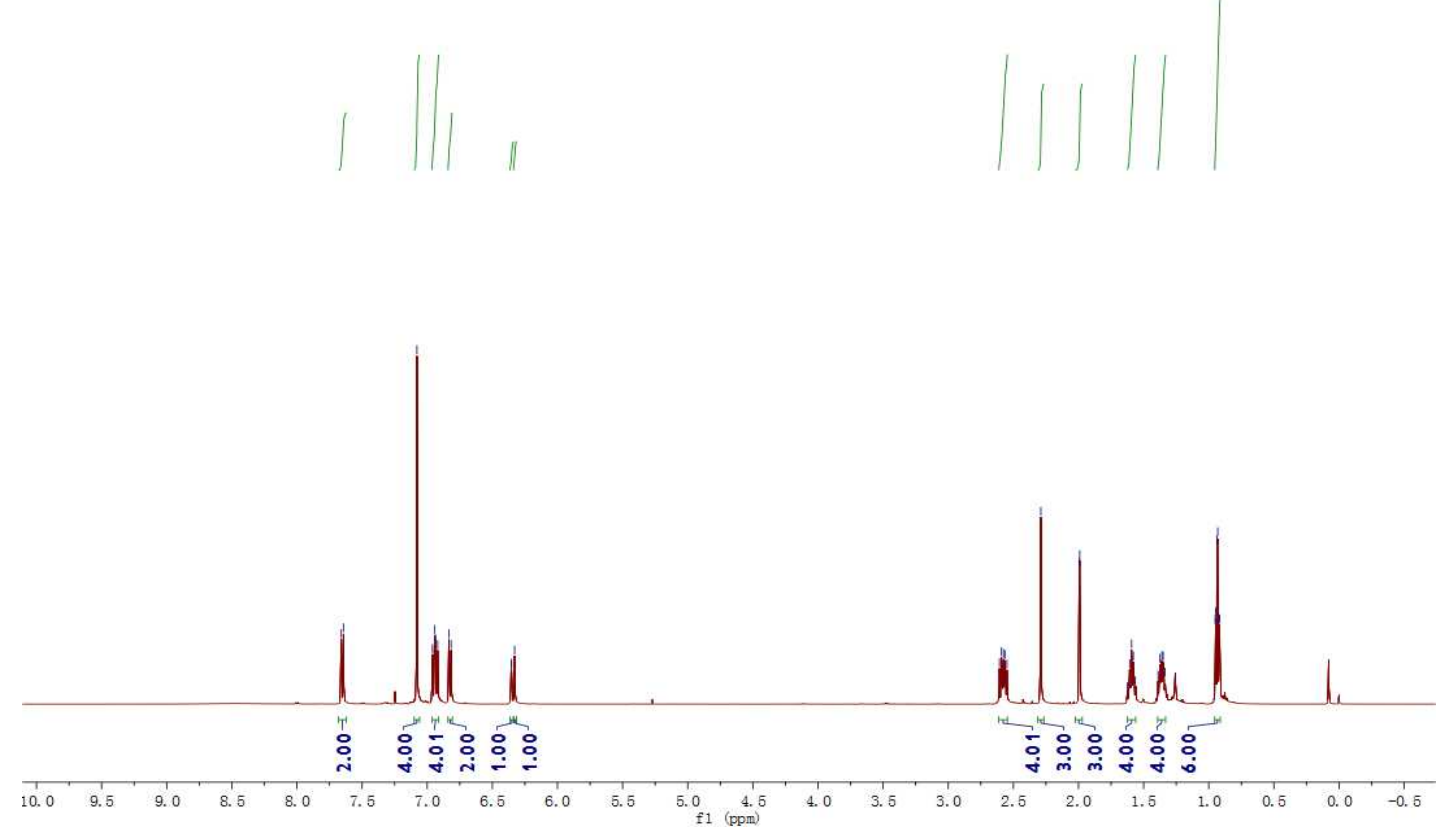

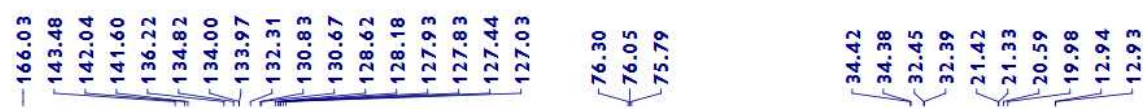

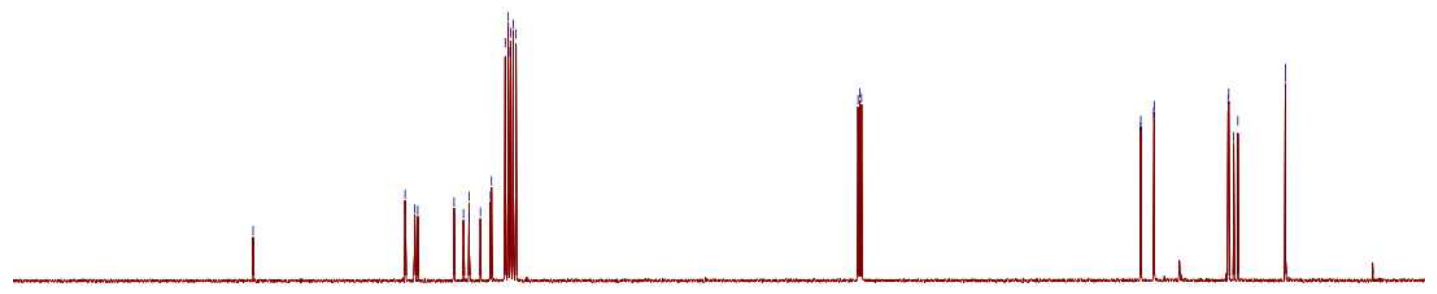

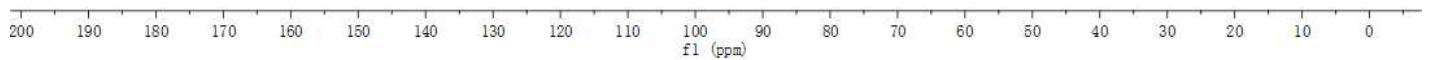


<smiles>[3H]NC(=O)C(C)=CC(=Cc1cccc(Br)c1)c1cccc(Br)c1</smiles>

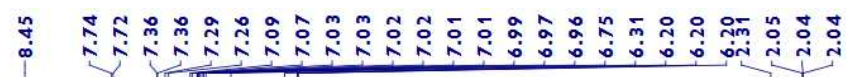
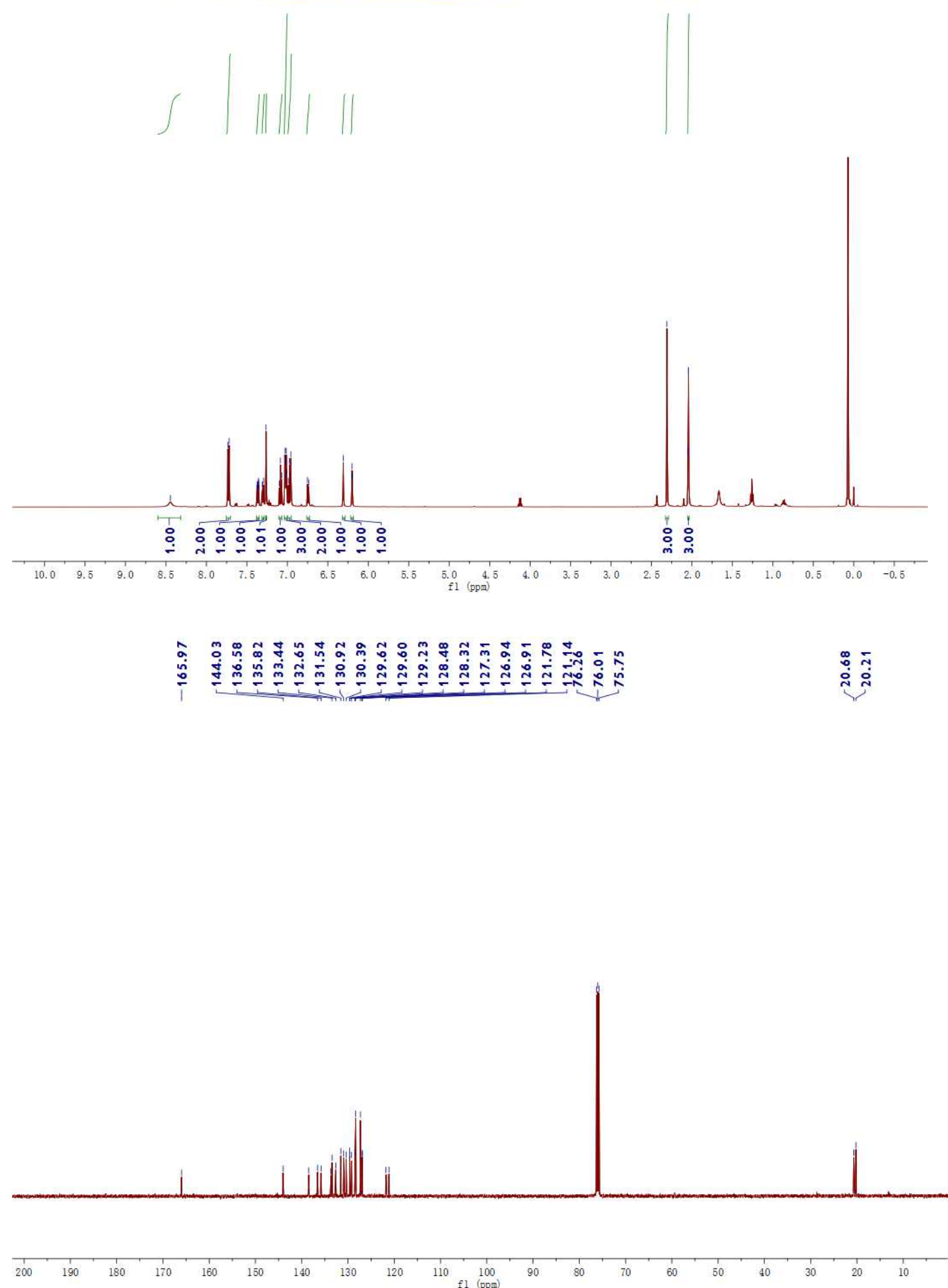

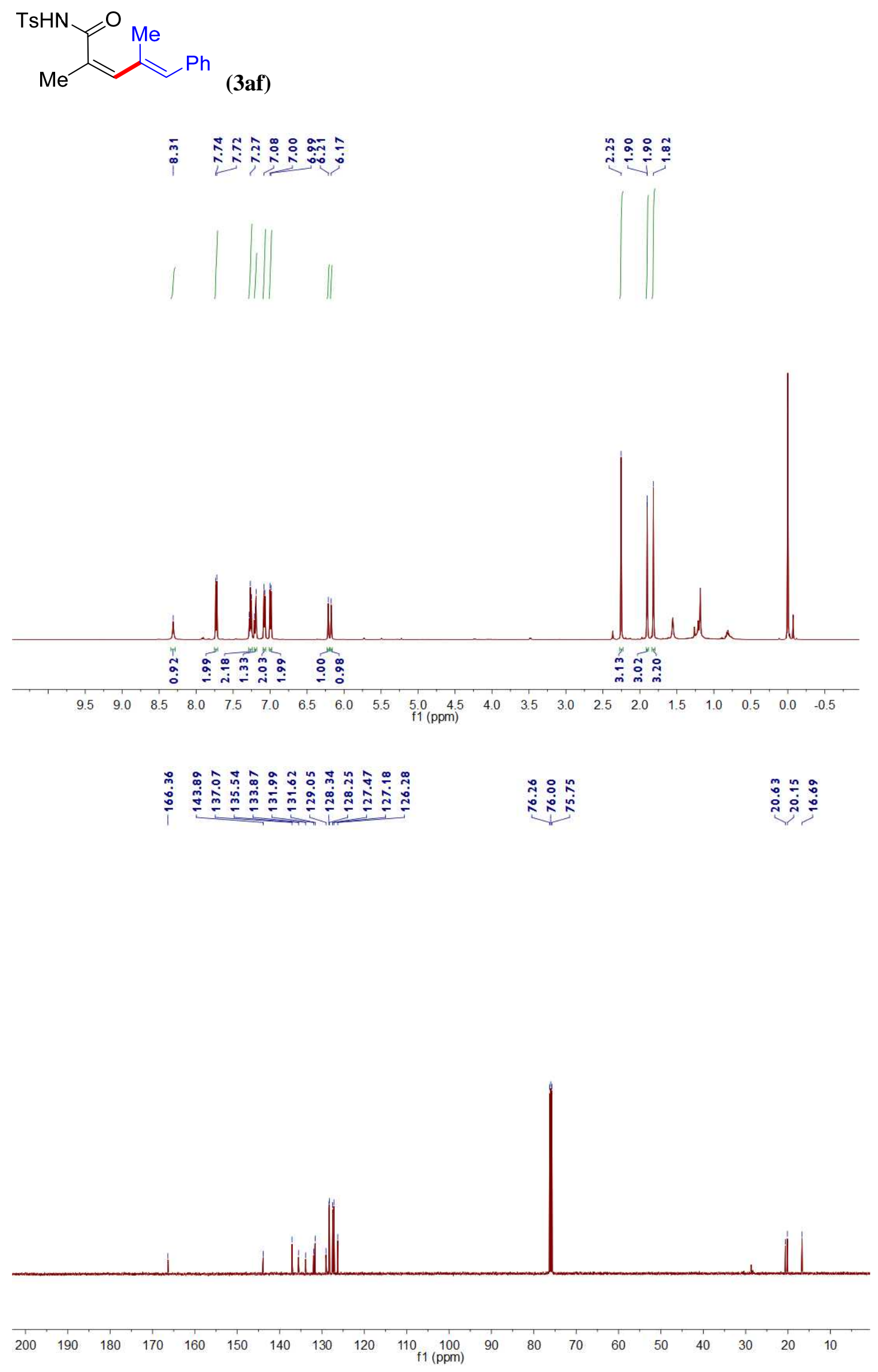


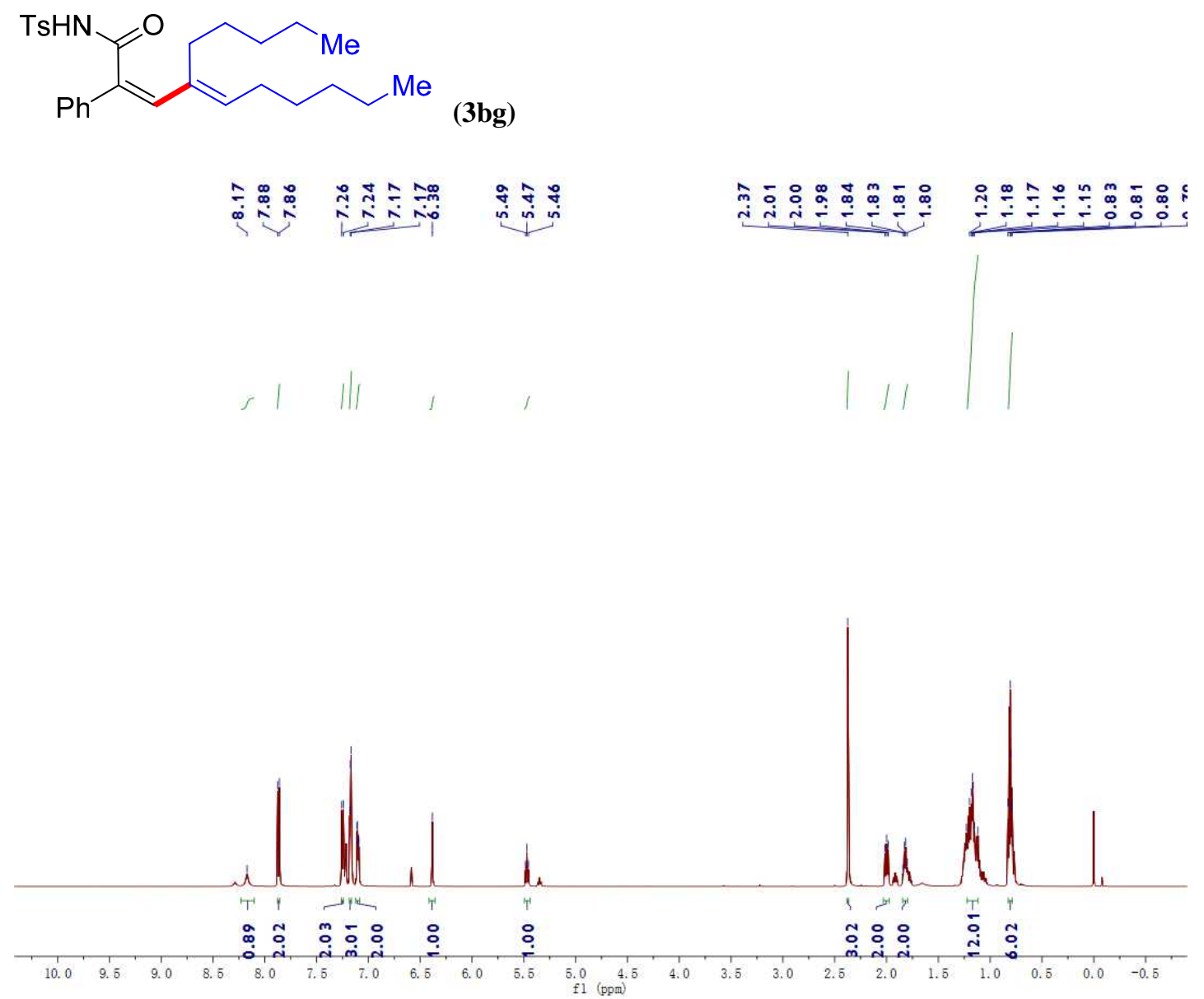

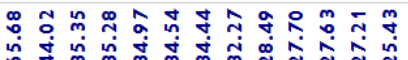

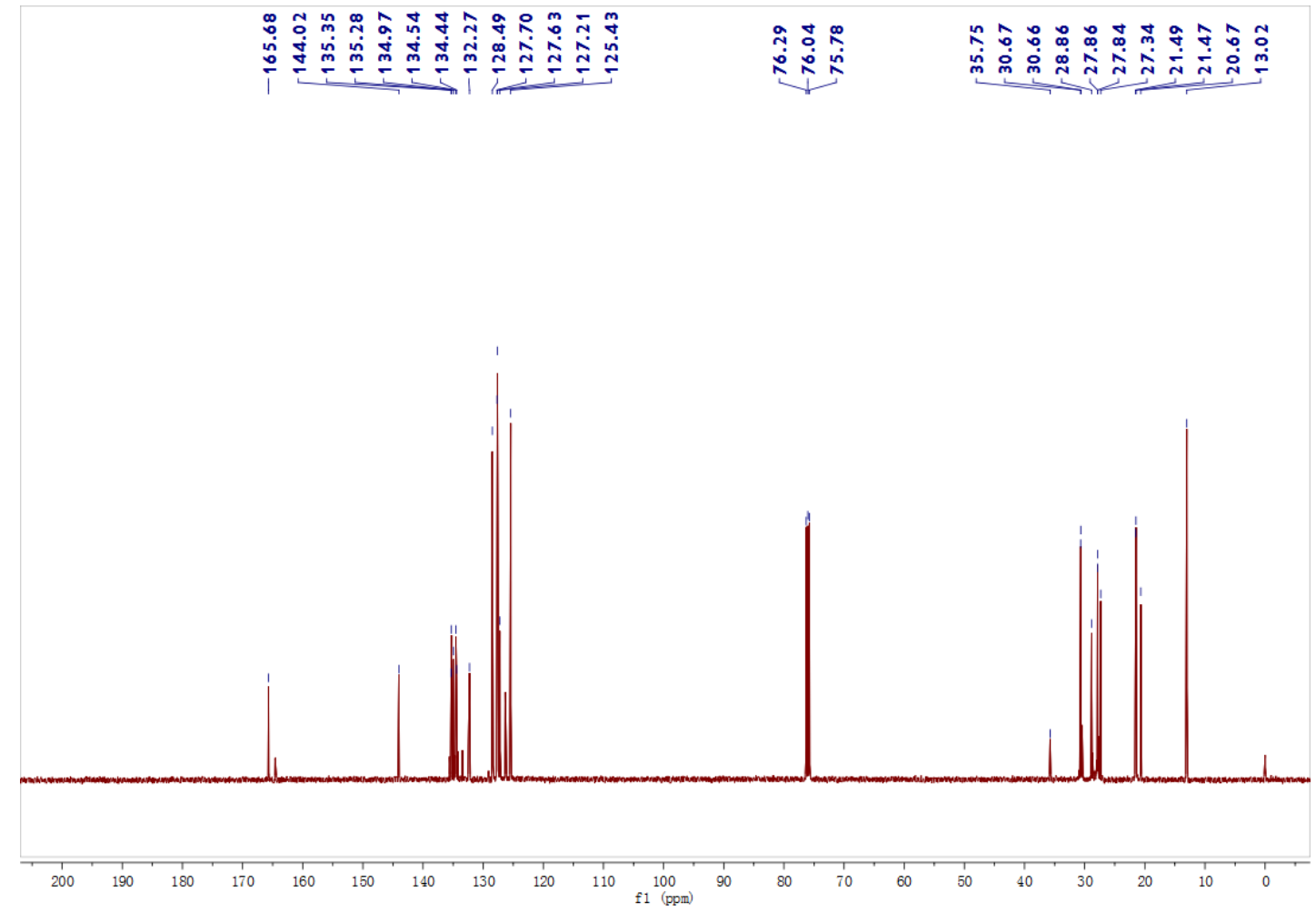




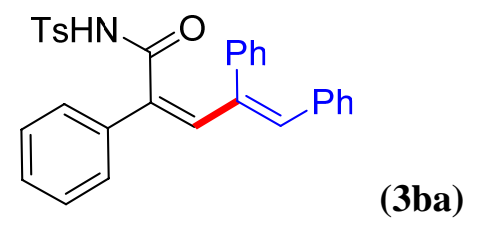

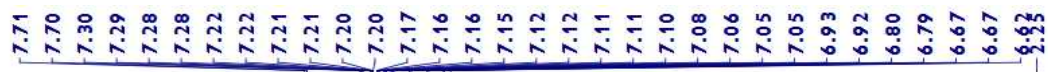

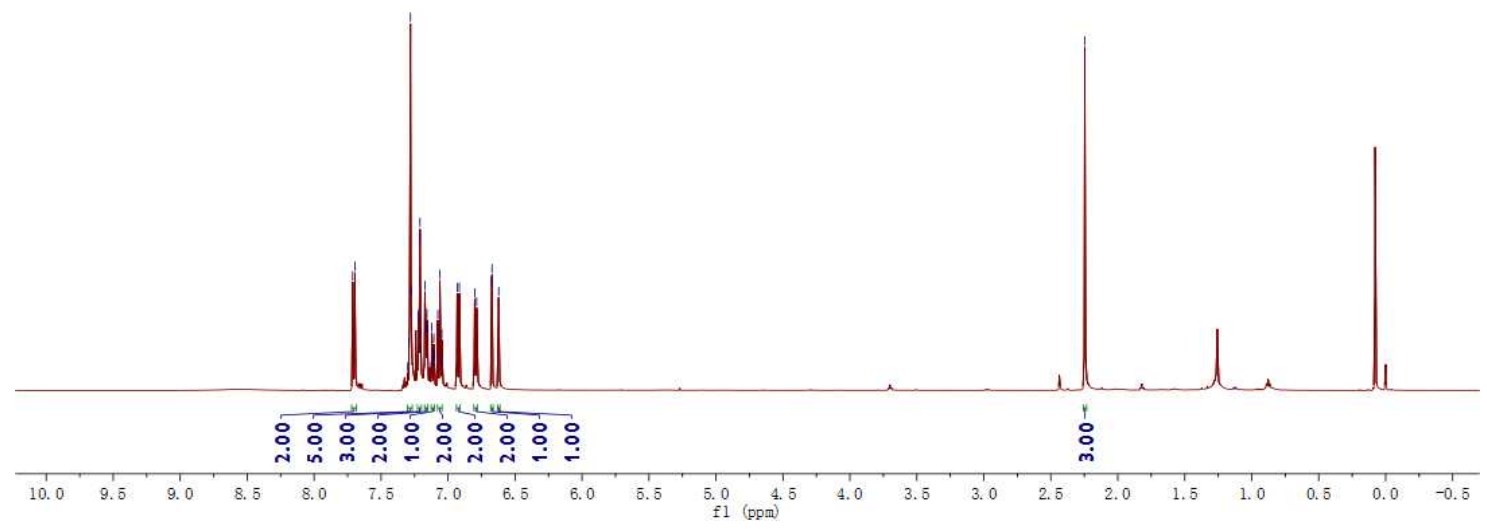

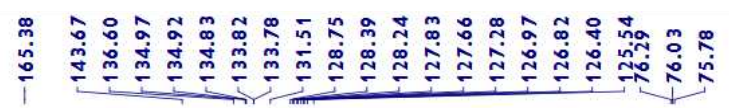

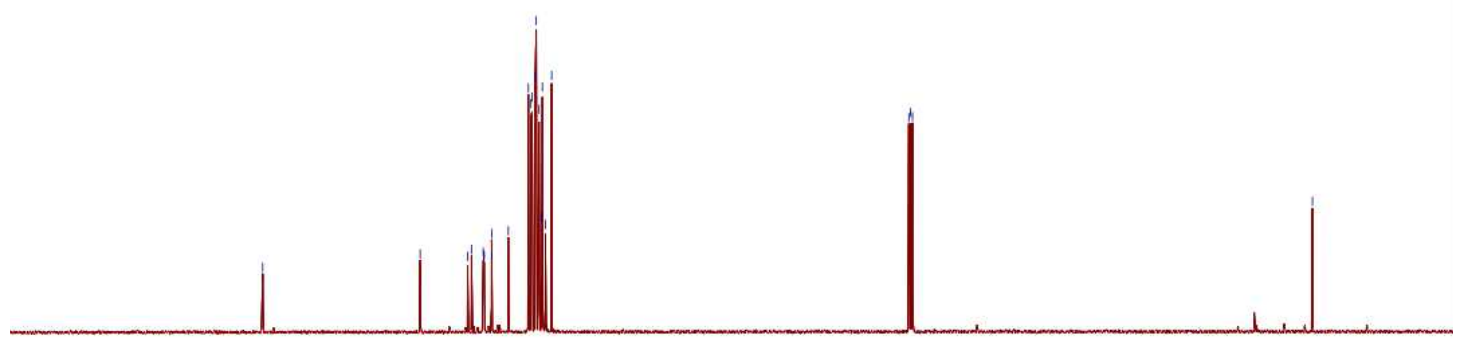

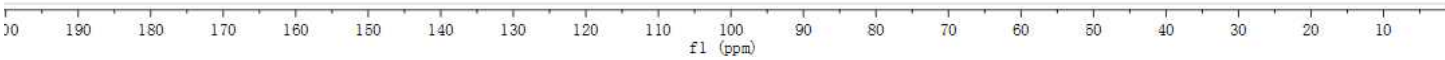


<smiles>COc1ccc(C(=CC(=Cc2ccccc2)c2ccccc2)C(=O)N[TeH])cc1</smiles>

(3ca)

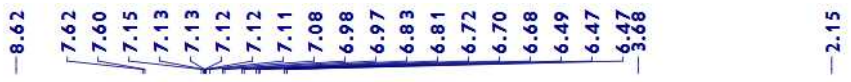

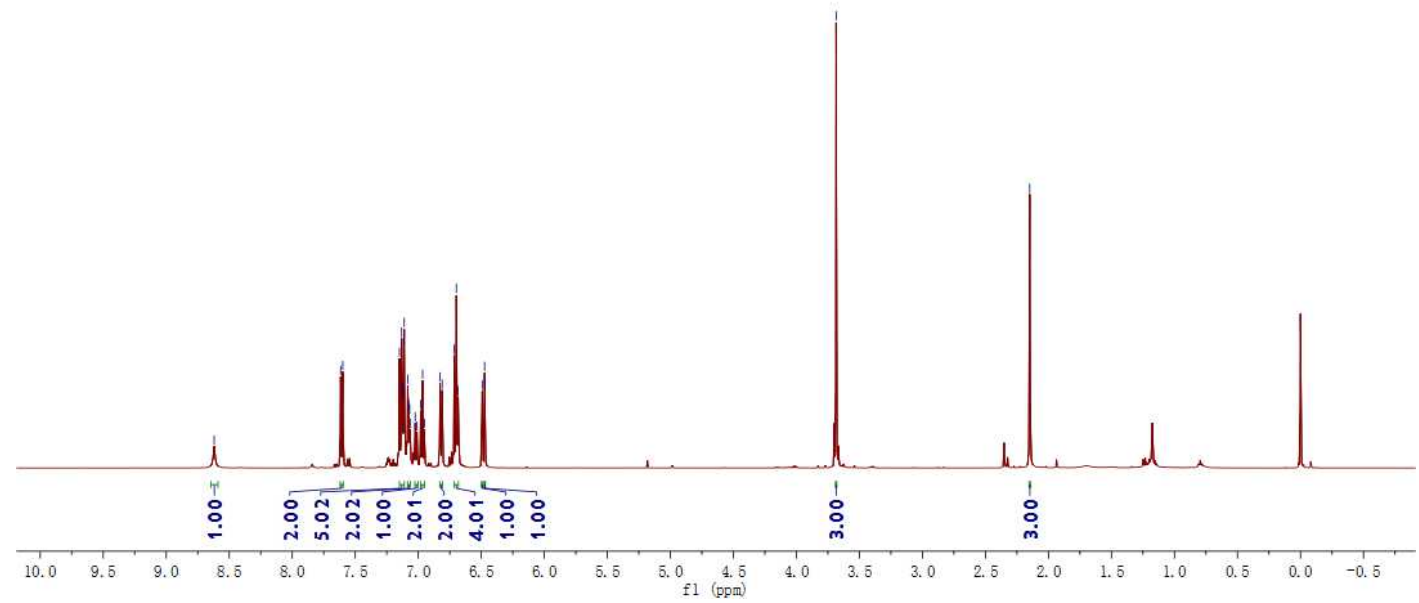

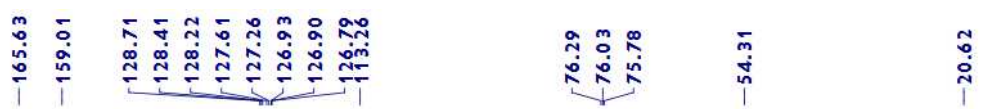

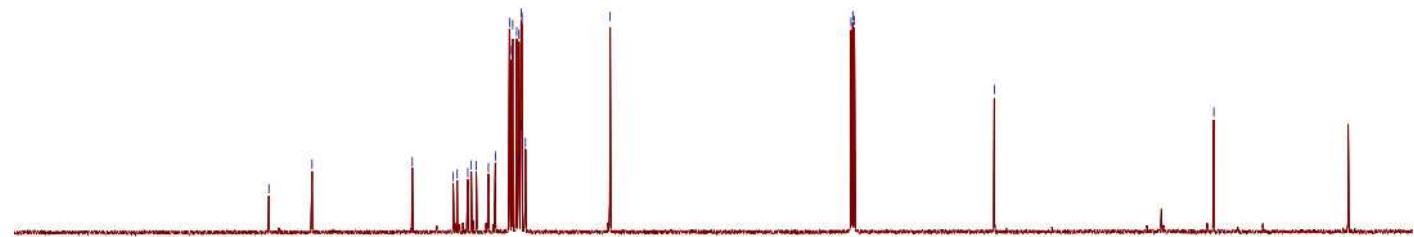

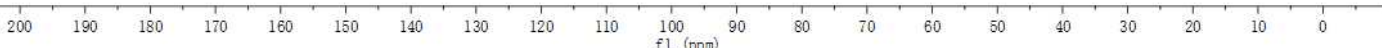


<smiles>O=C(N[Si])/C(=C\C(=C/c1ccccc1)c1ccccc1)c1ccc(C(F)(F)F)cc1</smiles>

(3da)
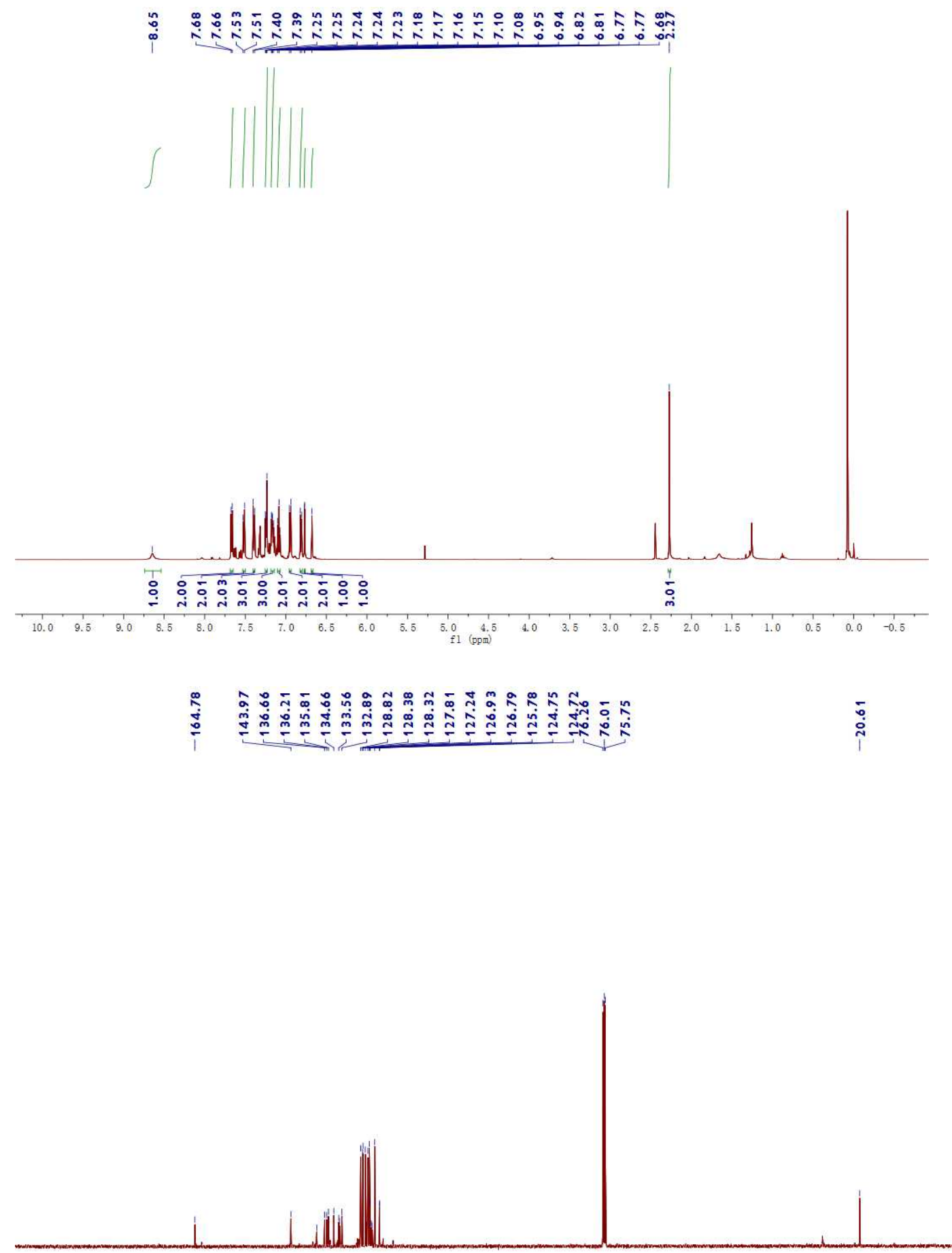

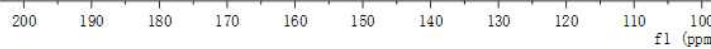


<smiles>O=C(N[AlH2])C(=CC(=Cc1ccccc1)c1ccc(F)cc1)c1ccccc1</smiles>

(3ea)

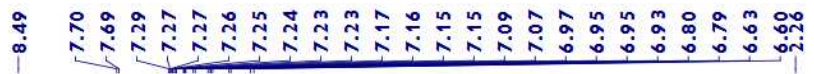
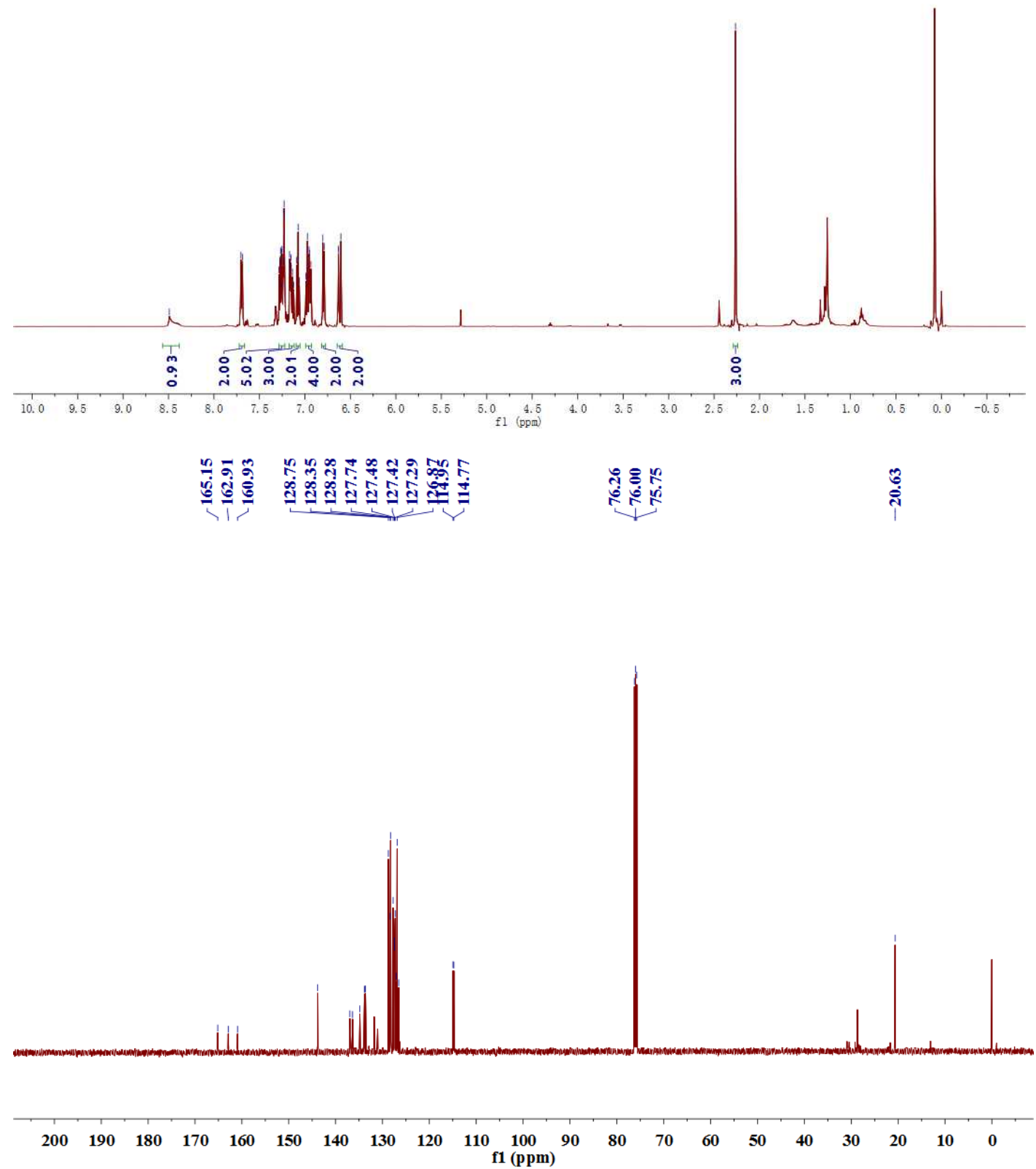
<smiles>O=C(N[AlH2])/C(=C\C(=C\c1ccccc1)c1ccccc1F)c1ccccc1</smiles>

店

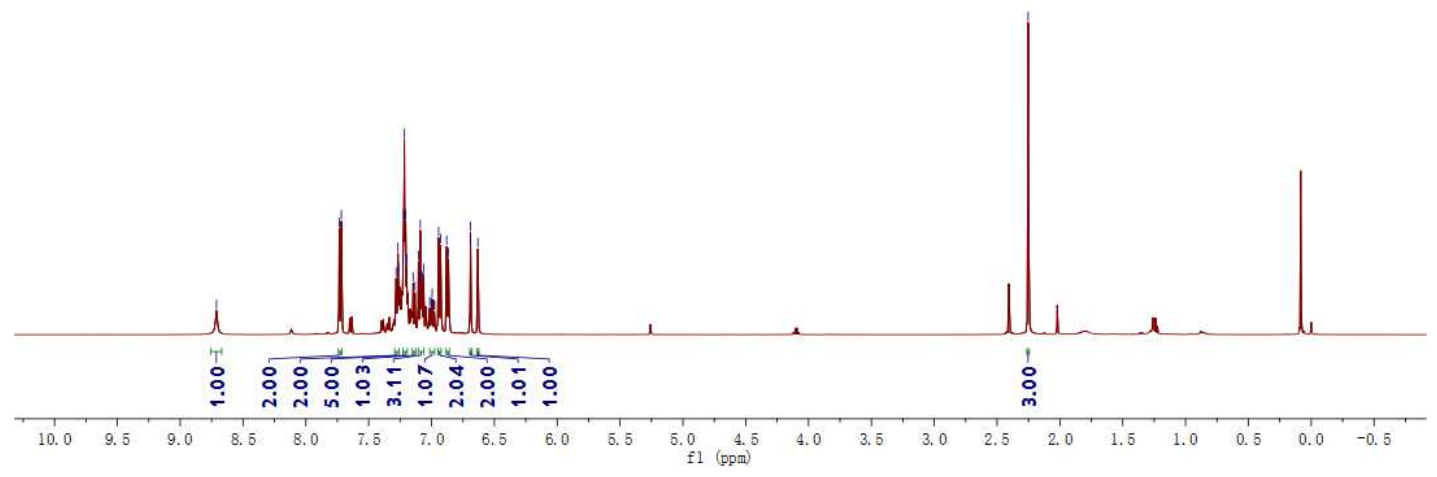

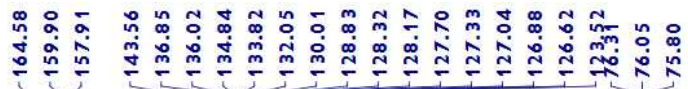

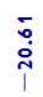

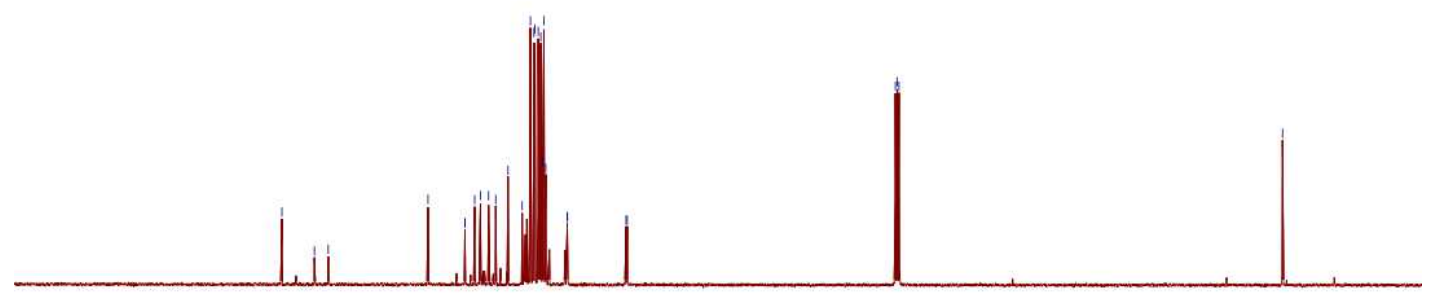

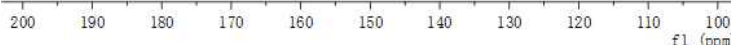


<smiles>CCCCCCCCNC(=O)C(=CC(=Cc1ccccc1)c1ccccc1)c1ccccc1</smiles>

(3ga)

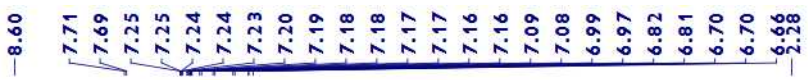

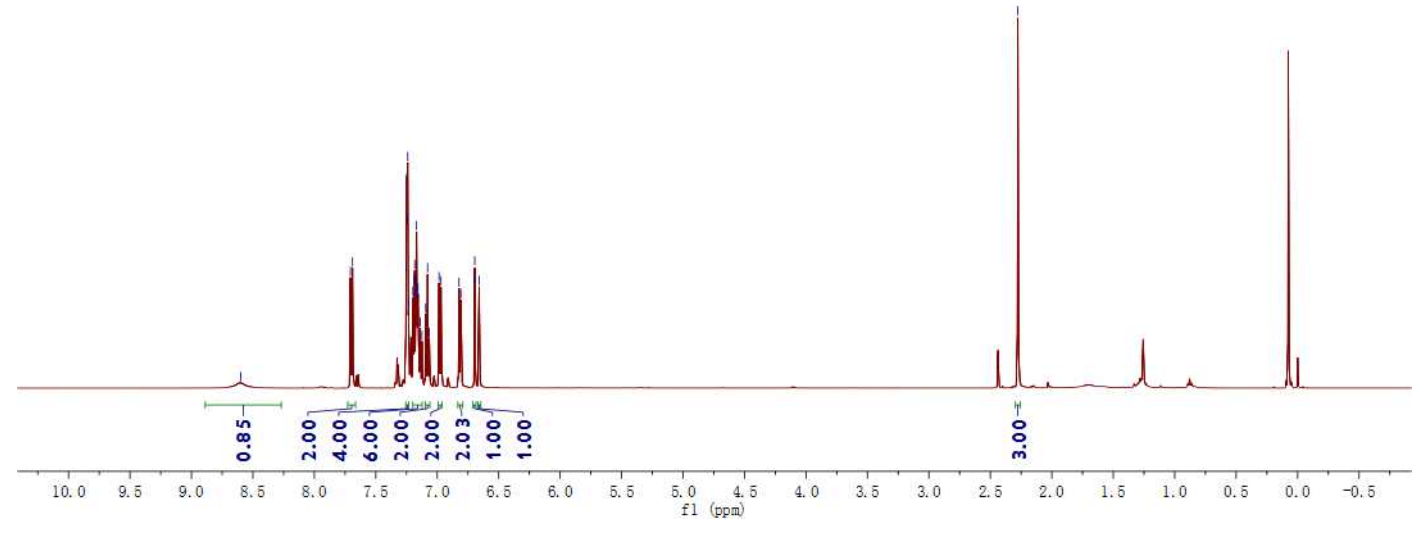

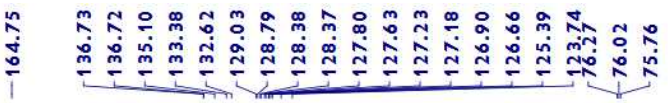

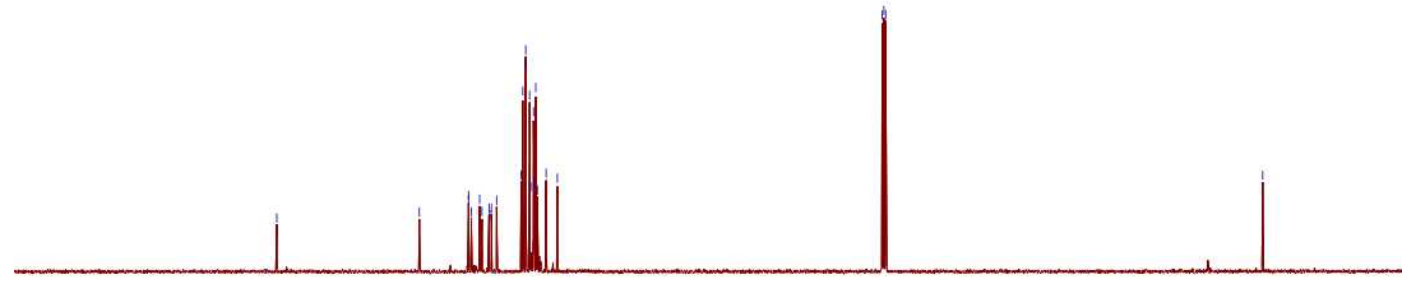

$\begin{array}{lllllllllll}1 & 1 & 1 & 1 & 1 & 1 & 1 & 1 & 1 & 1 & 1 \\ 200 & 190 & 180 & 170 & 160 & 150 & 140 & 130 & 120 & 110 & 100 \\ \text { f1 } & (\mathrm{pgm})\end{array}$ 
<smiles>[CH]NC(=O)C(=CC(=Cc1ccccc1)Cc1ccccc1)c1ccccc1</smiles>
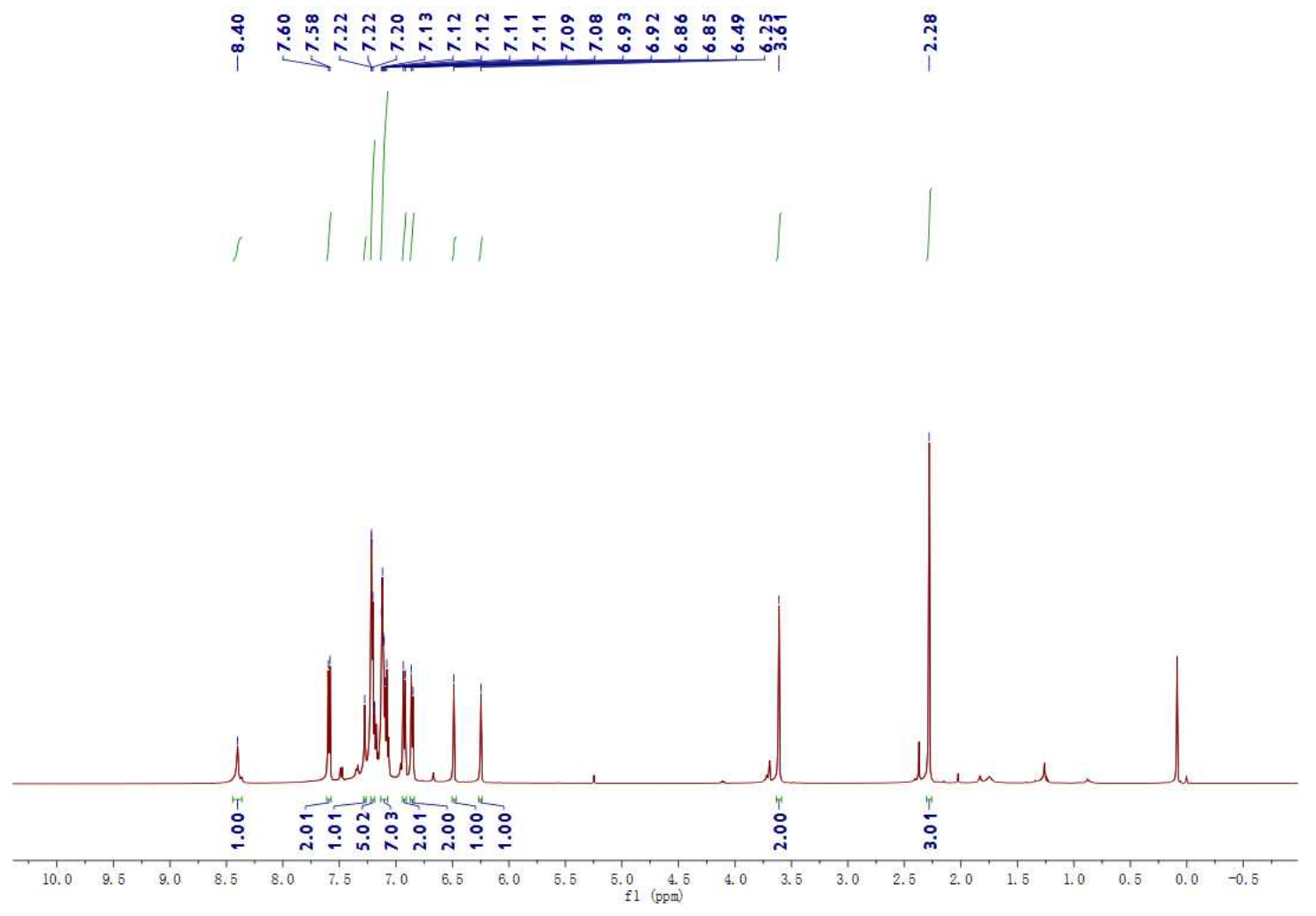

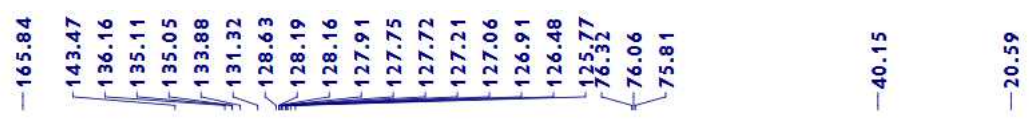

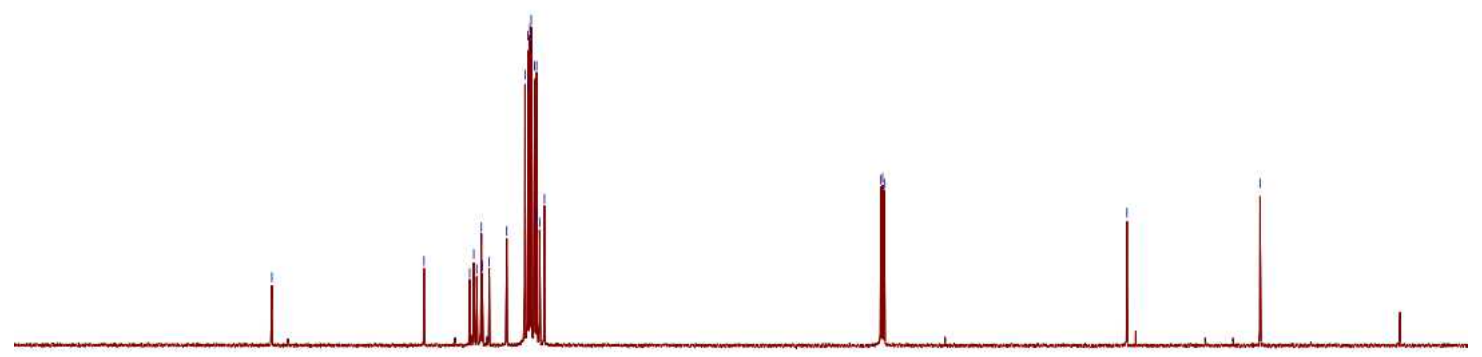

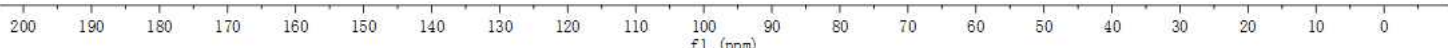


<smiles>CCCCCC/C(=C/C(=C/c1ccccc1)c1ccccc1)C(=O)N[AlH2]</smiles>

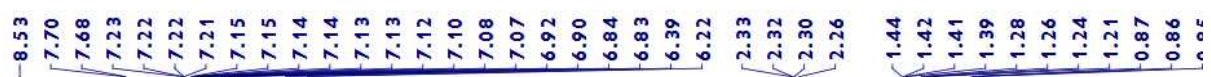

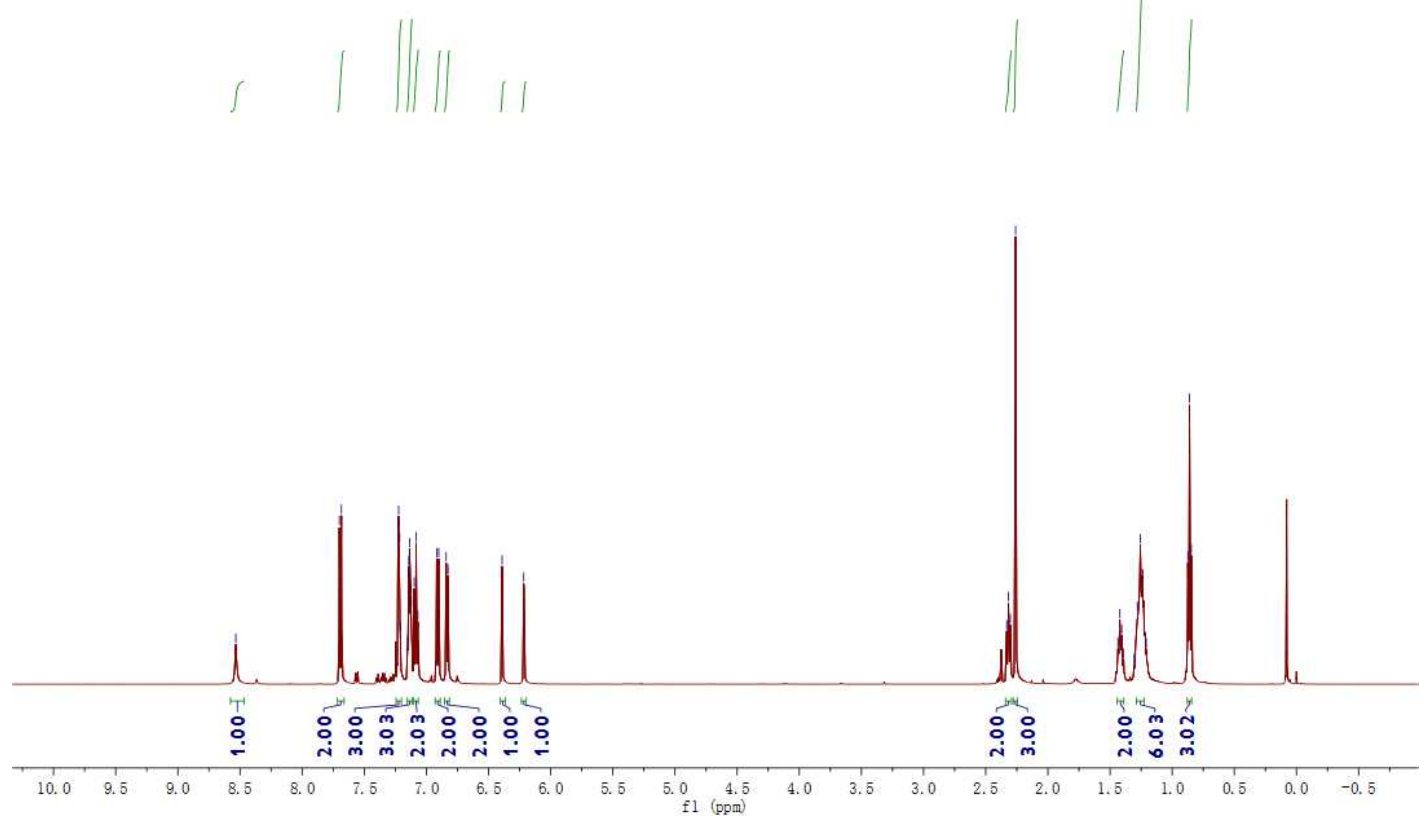

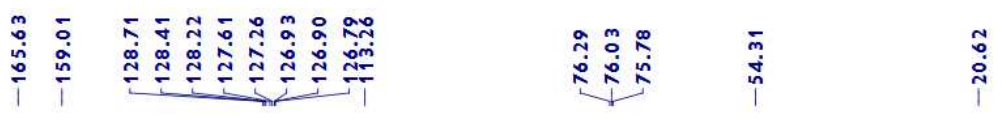

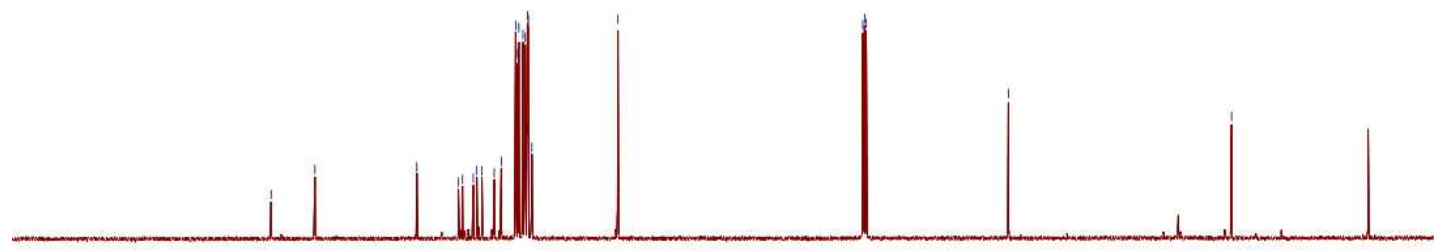

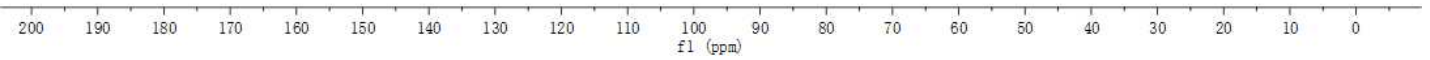




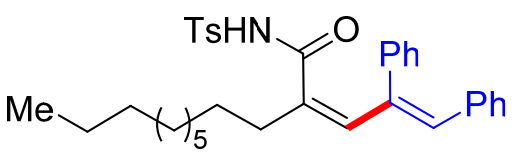

(3ja)

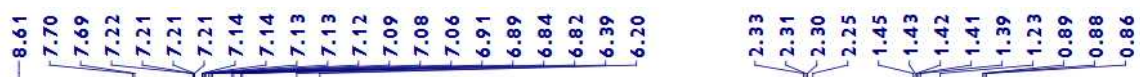

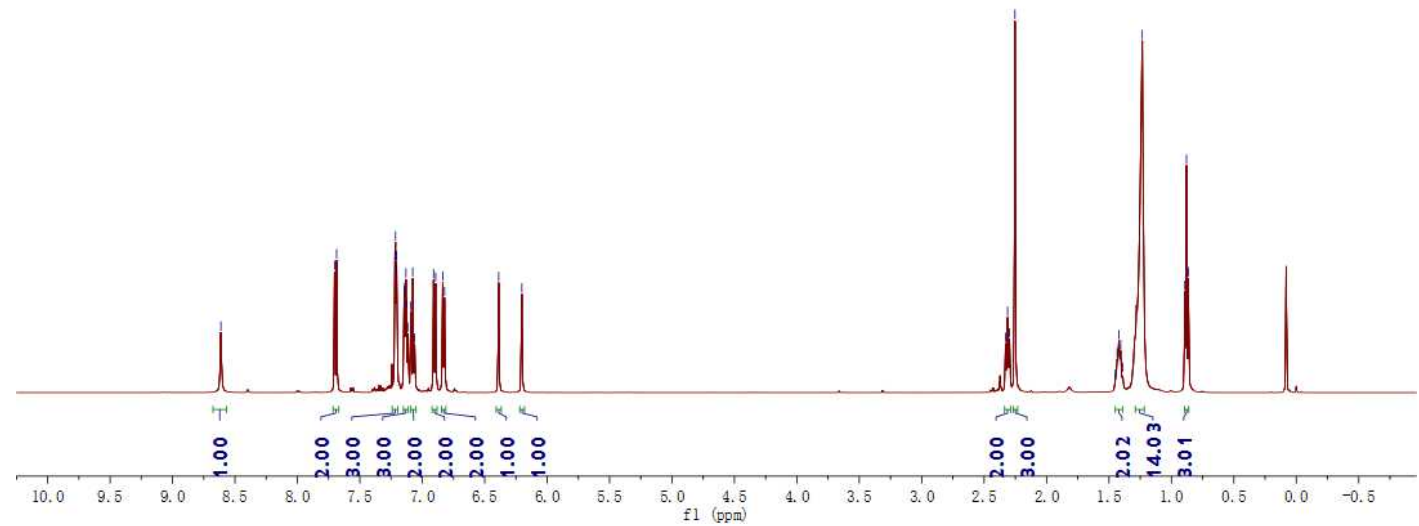

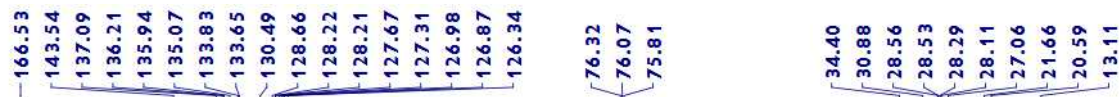

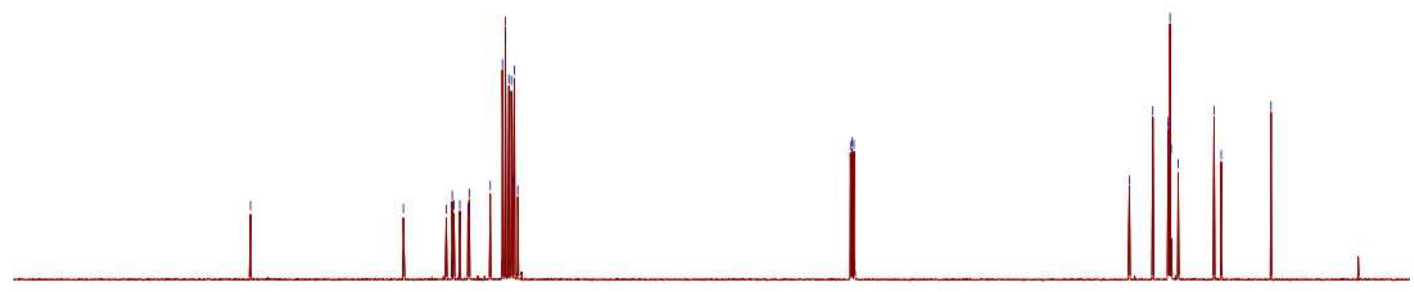

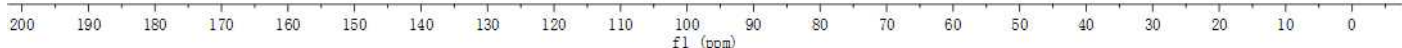




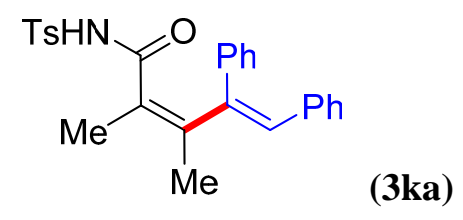

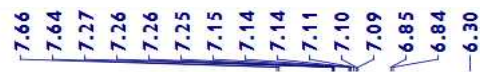

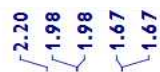
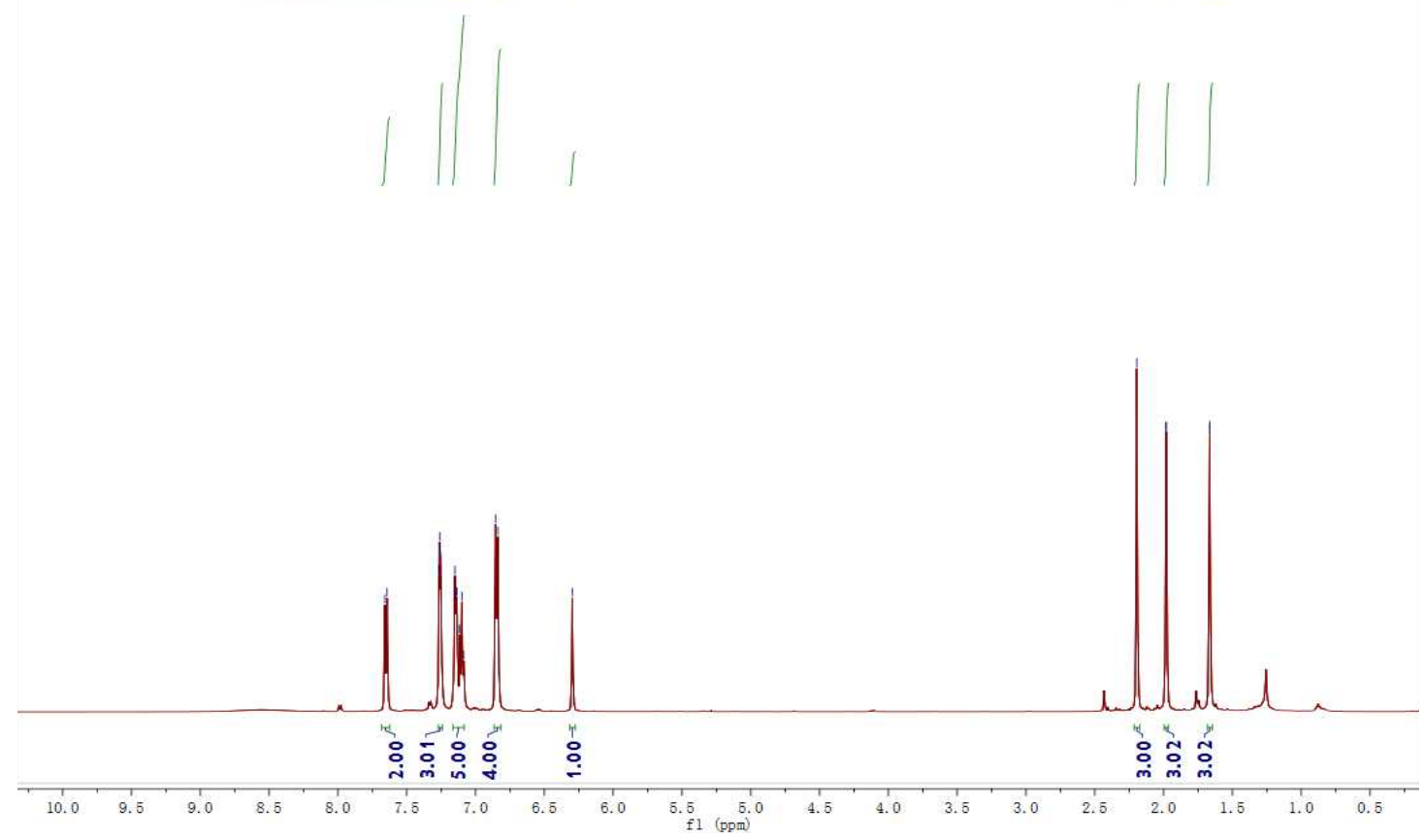

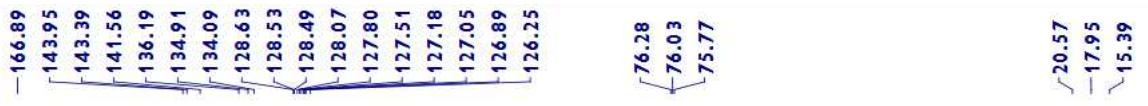

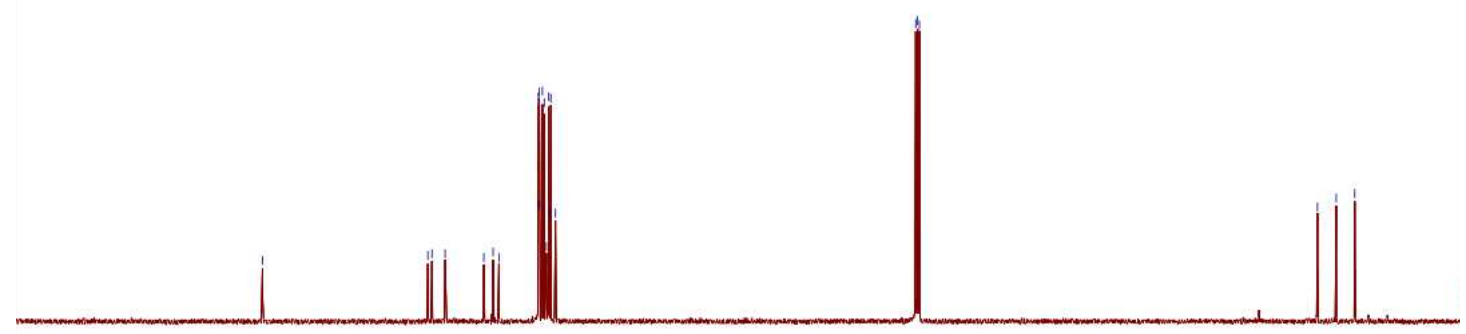

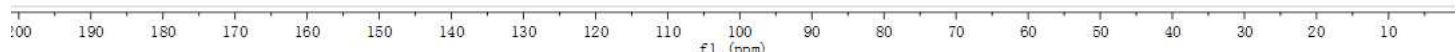




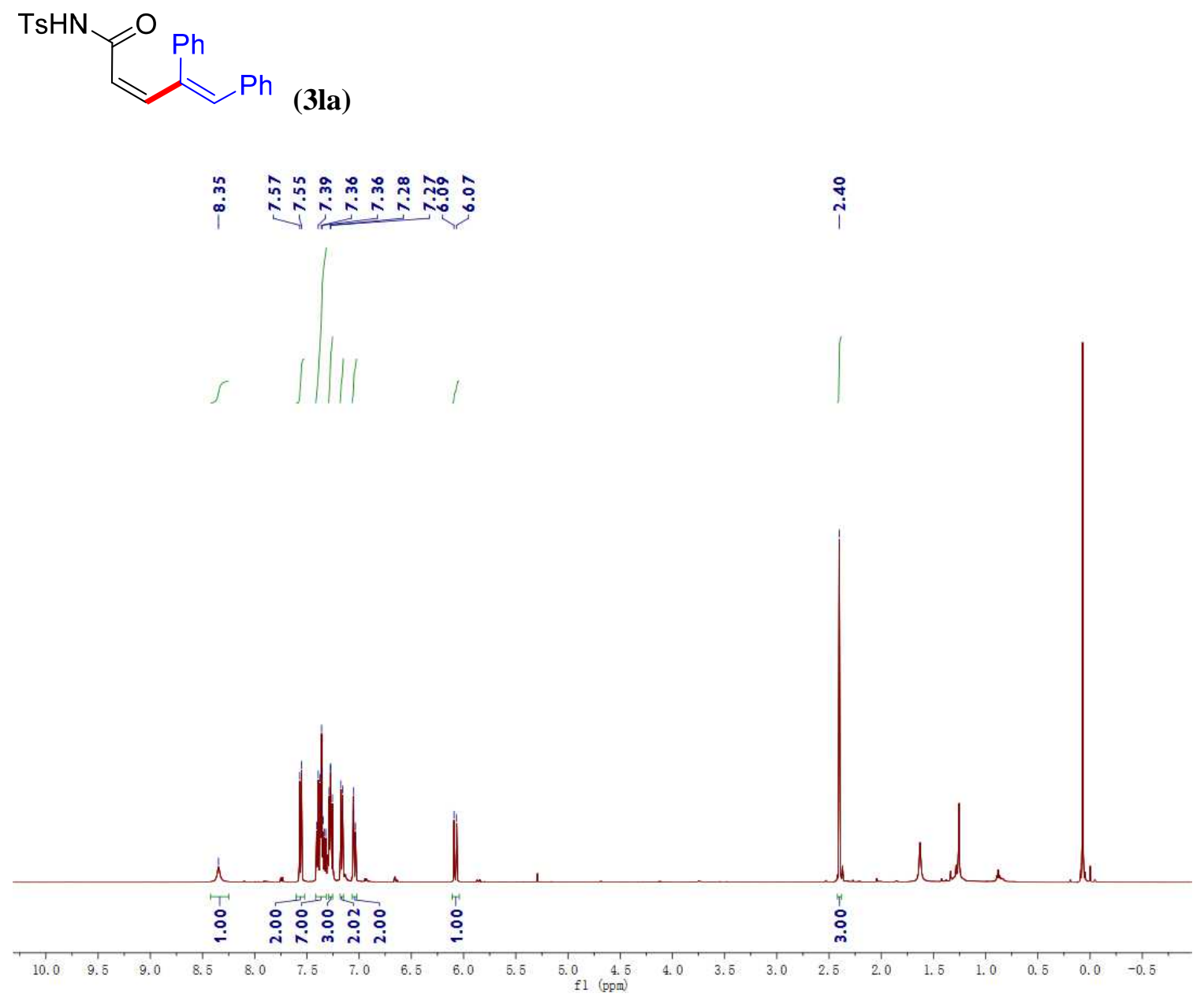

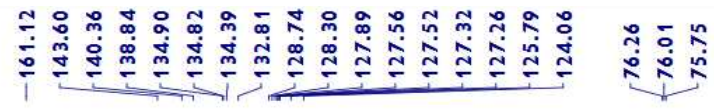

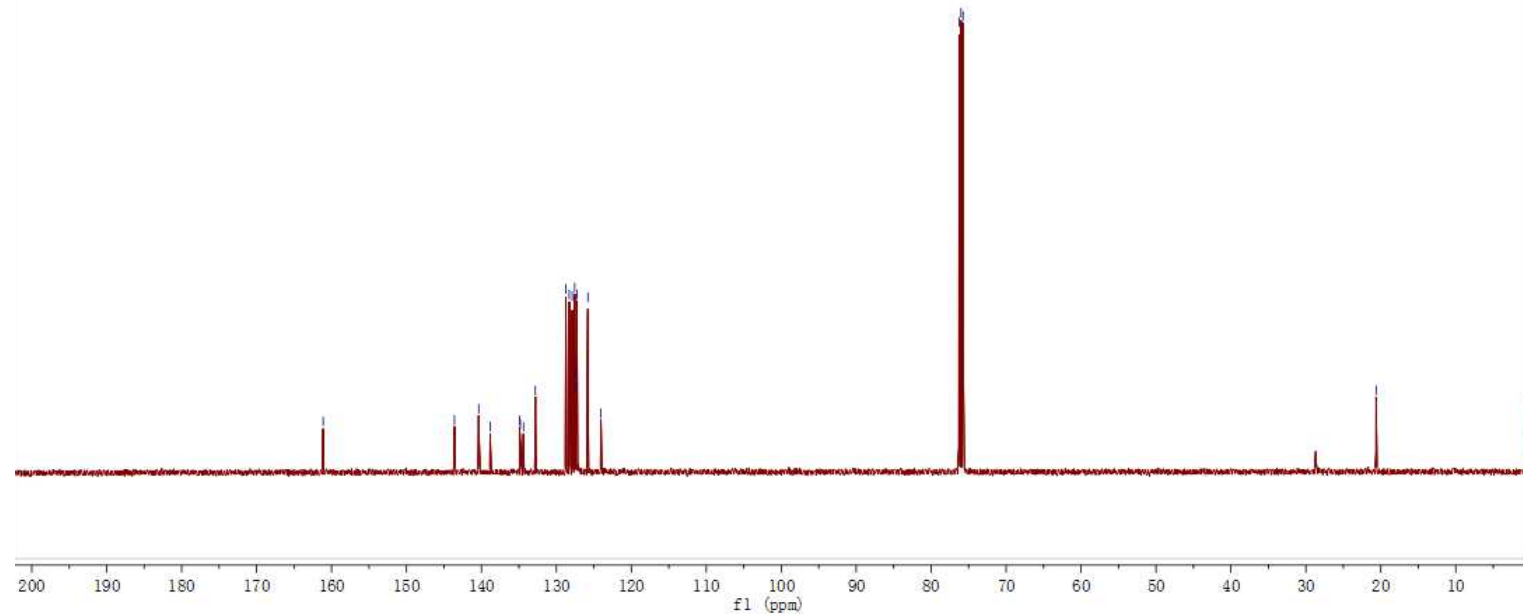



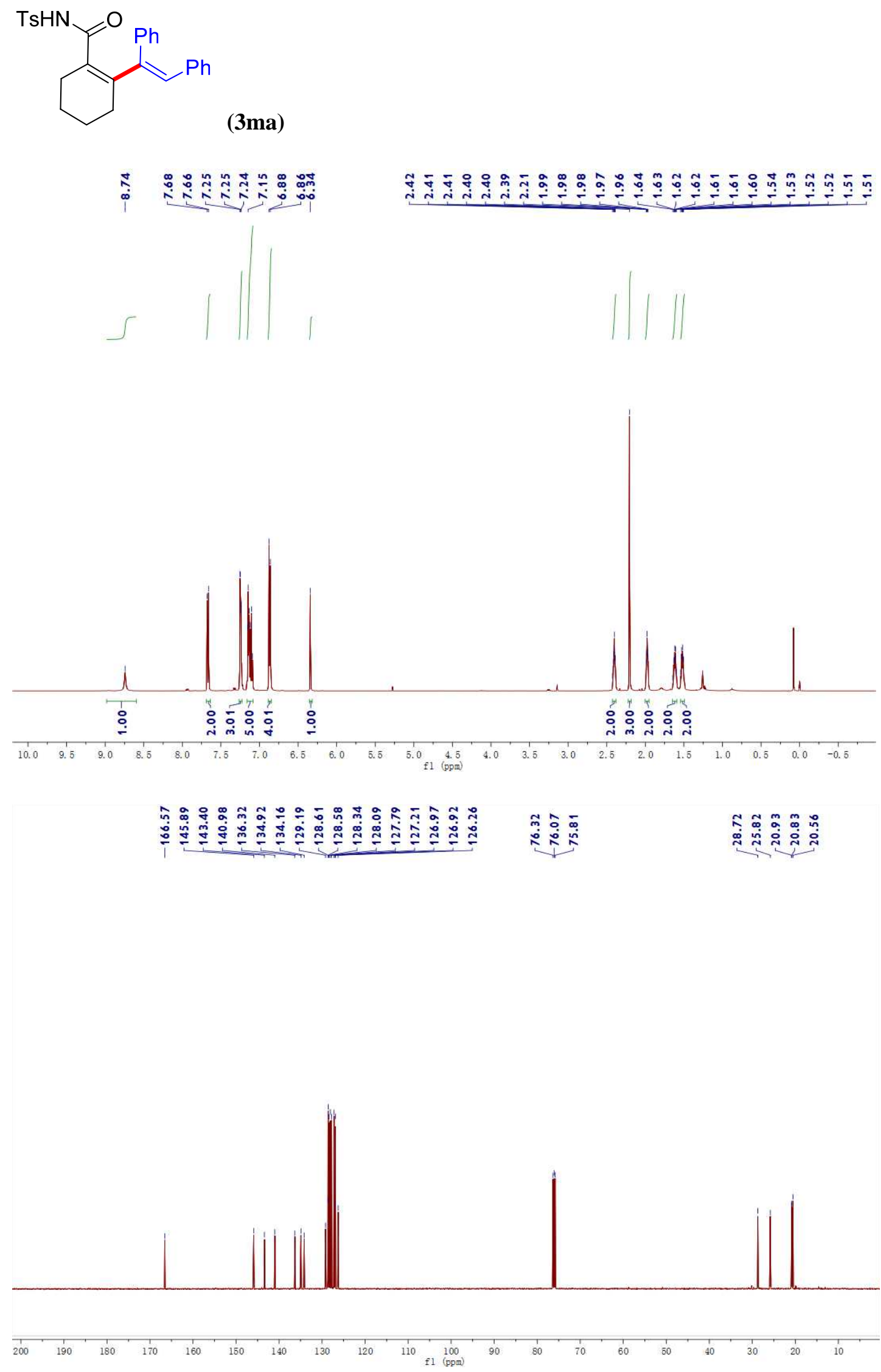


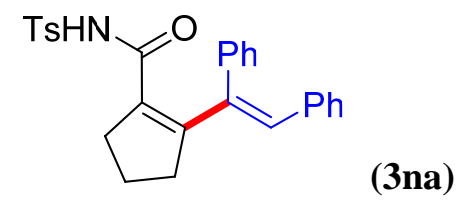

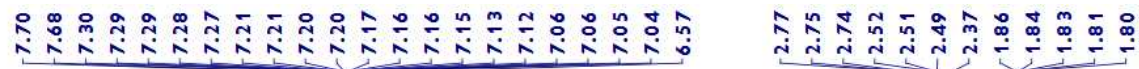
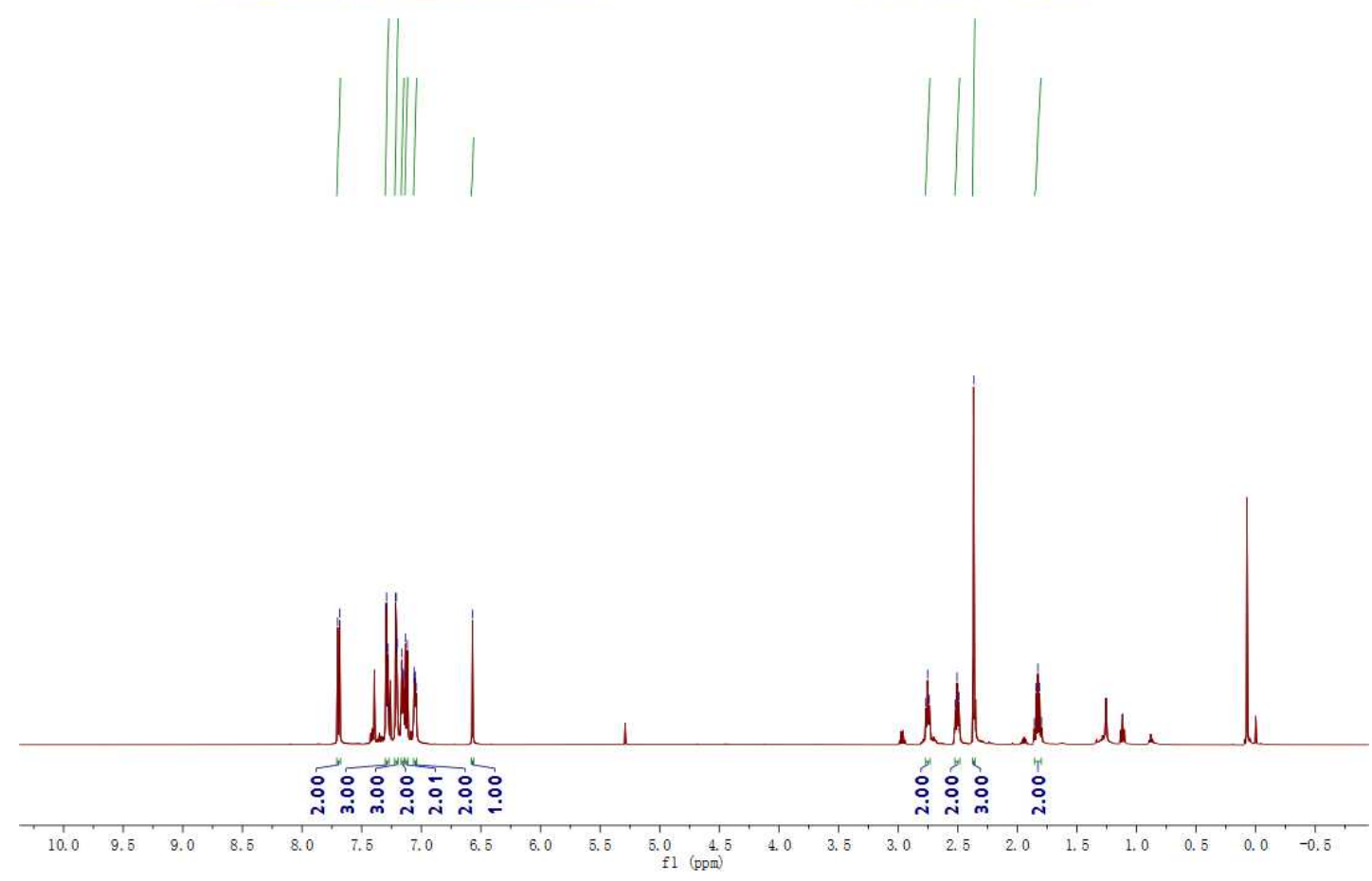

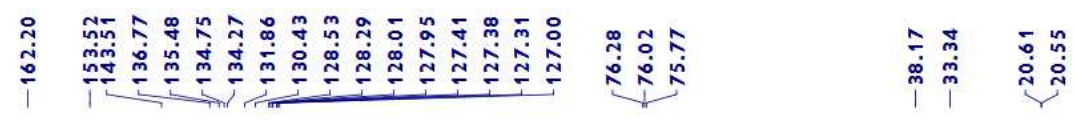

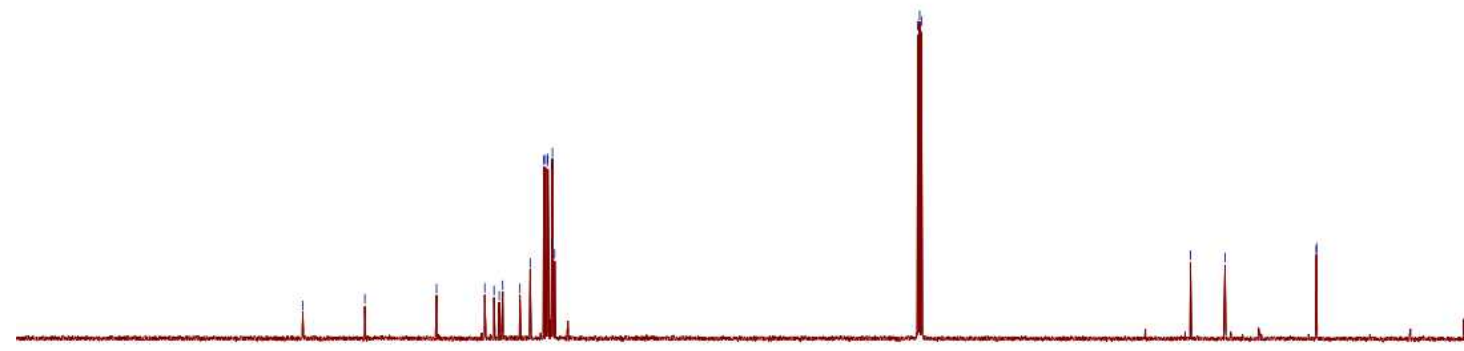

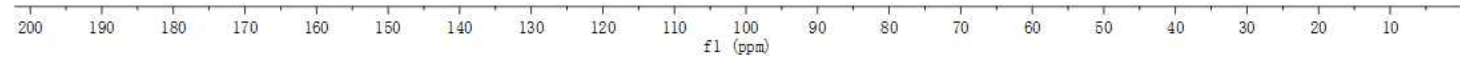



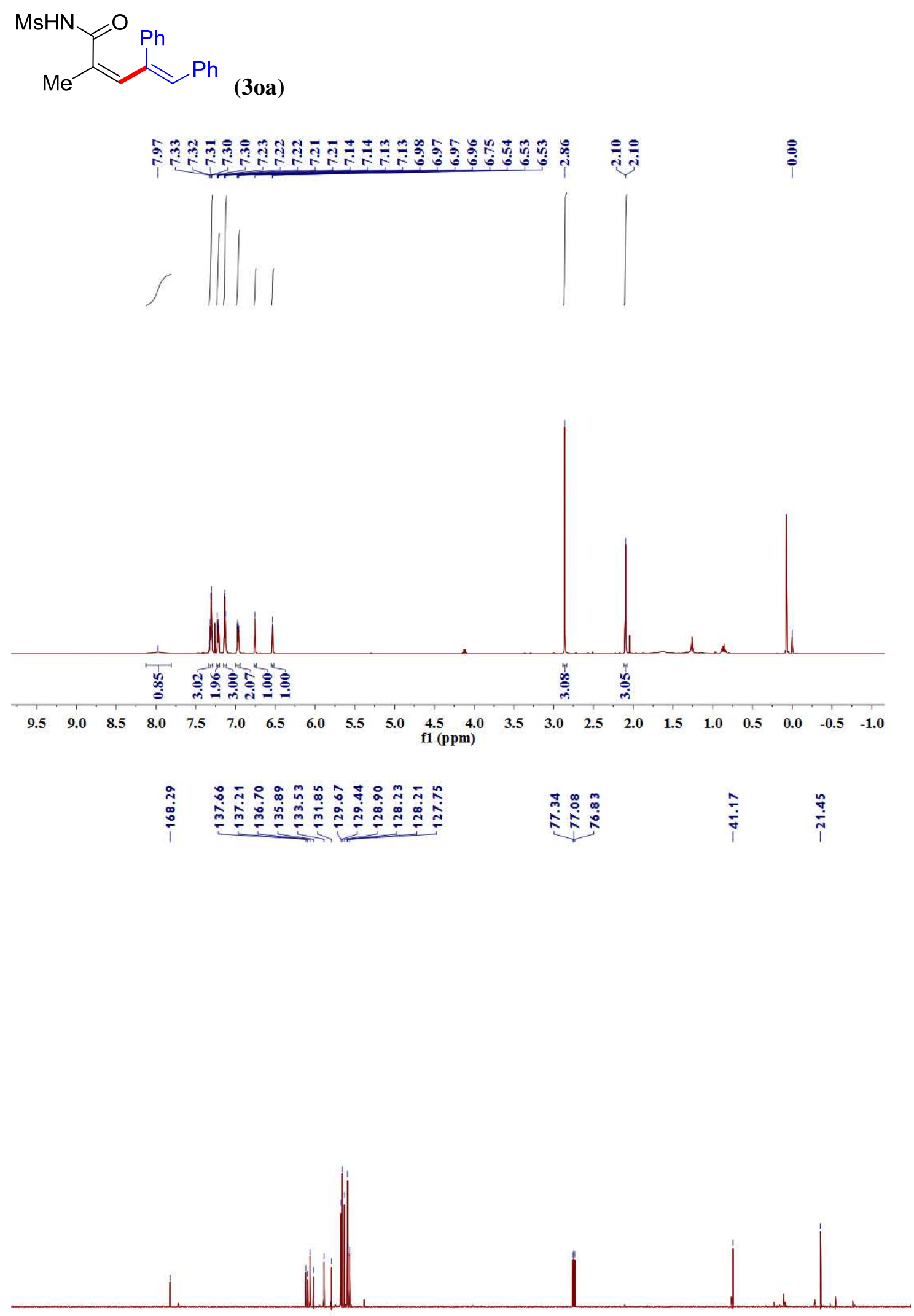

$\begin{array}{llllllllllllllllllllll}200 & 190 & 180 & 170 & 160 & 150 & 140 & 130 & 120 & 110 & 100 & 90 & 80 & 70 & 60 & 50 & 40 & 30 & 20 & 10\end{array}$ 
<smiles>C=C(C)[C@H]1CCC(C(=O)N[Sb])=C(/C(=C/c2ccccc2)c2ccccc2)C1</smiles>

(3pa)

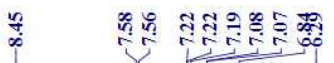

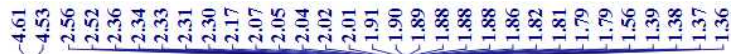
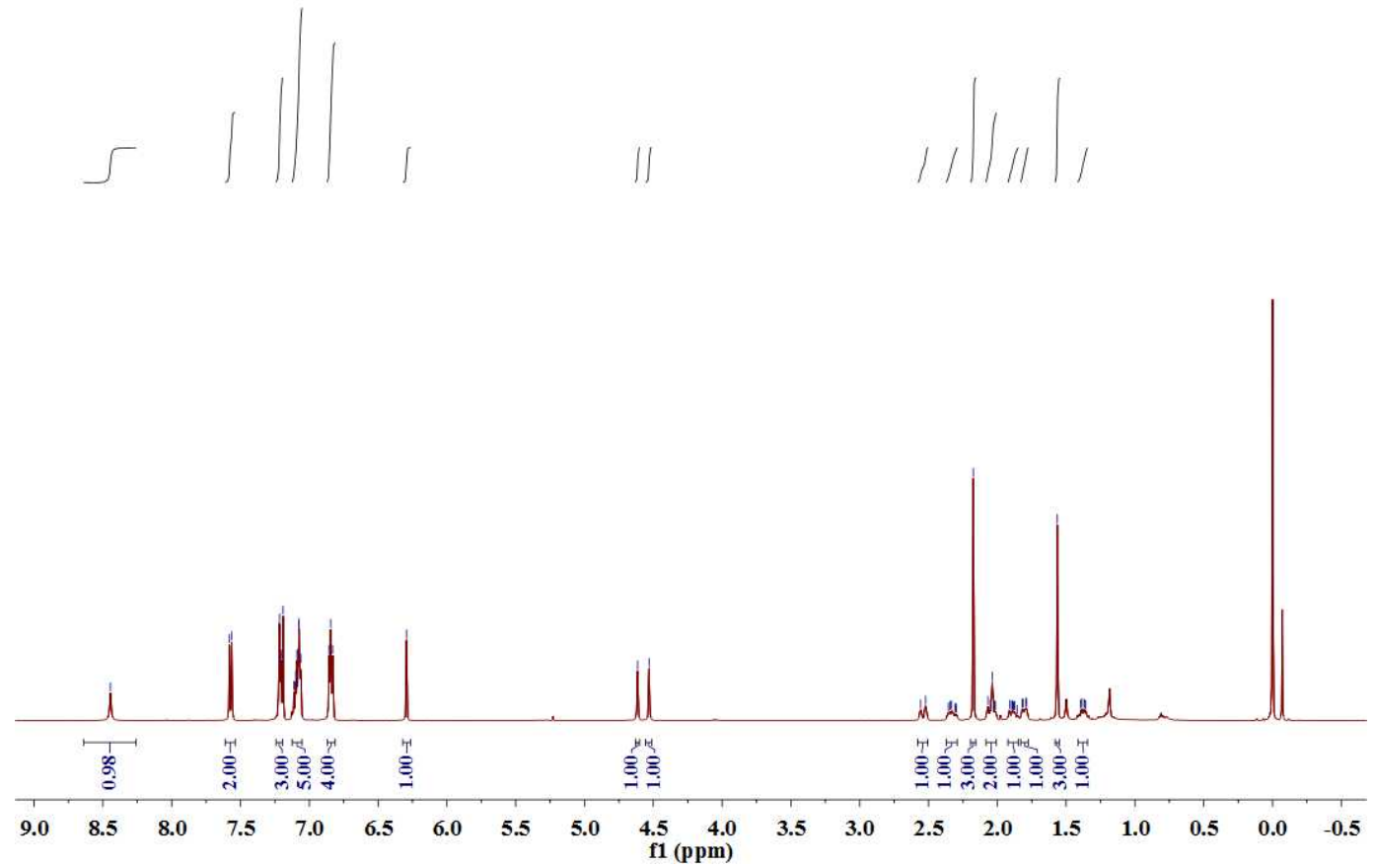

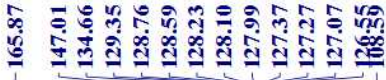

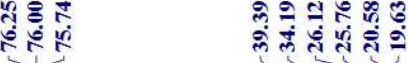

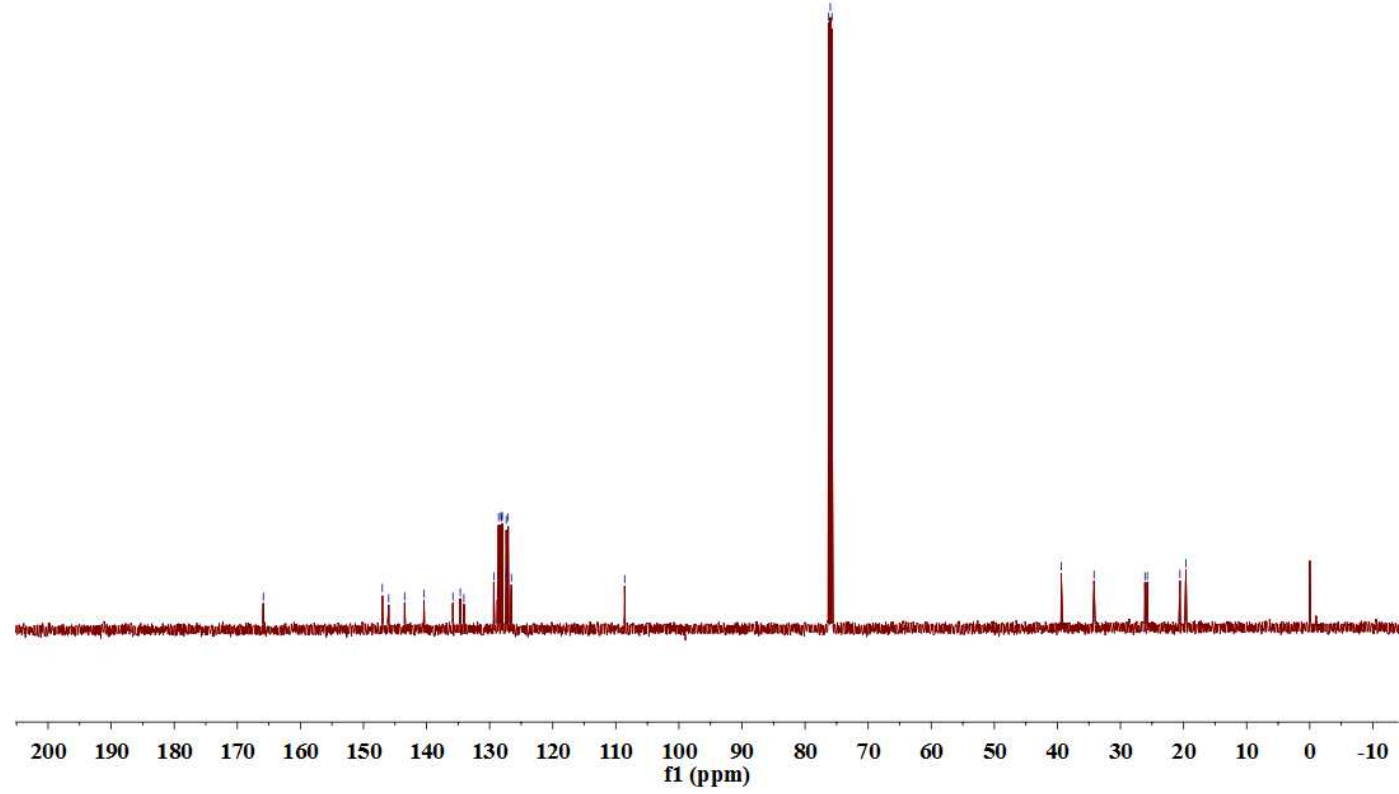


<smiles>C=C(C)[C@H]1CCC(C(N)=O)=C(CCCCCC)C1=CCCCC</smiles>

(3pg)

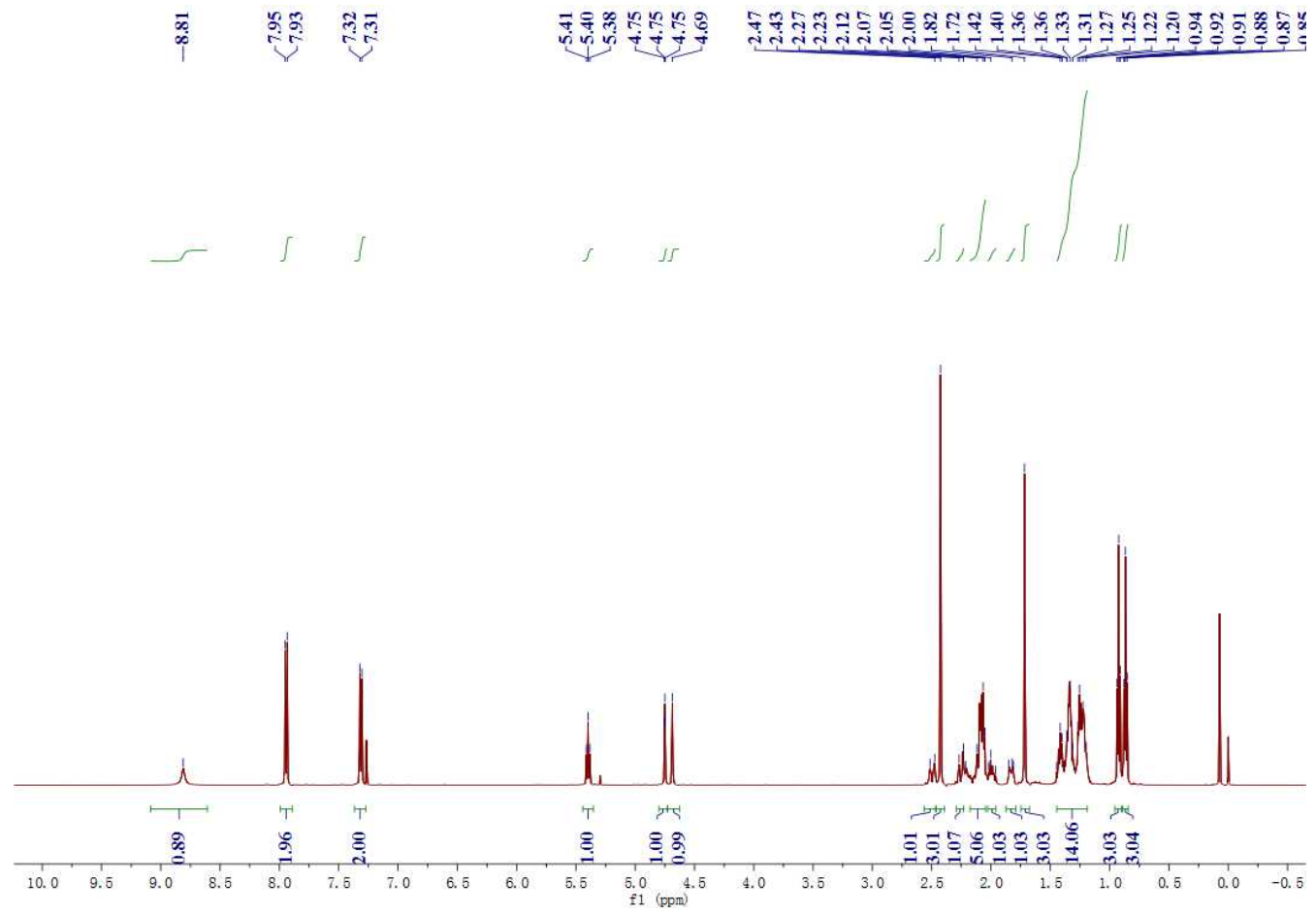

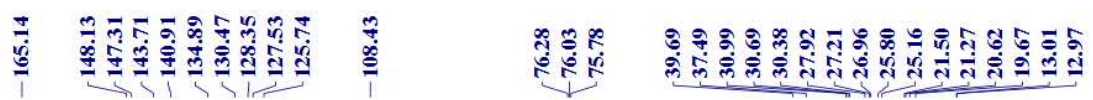

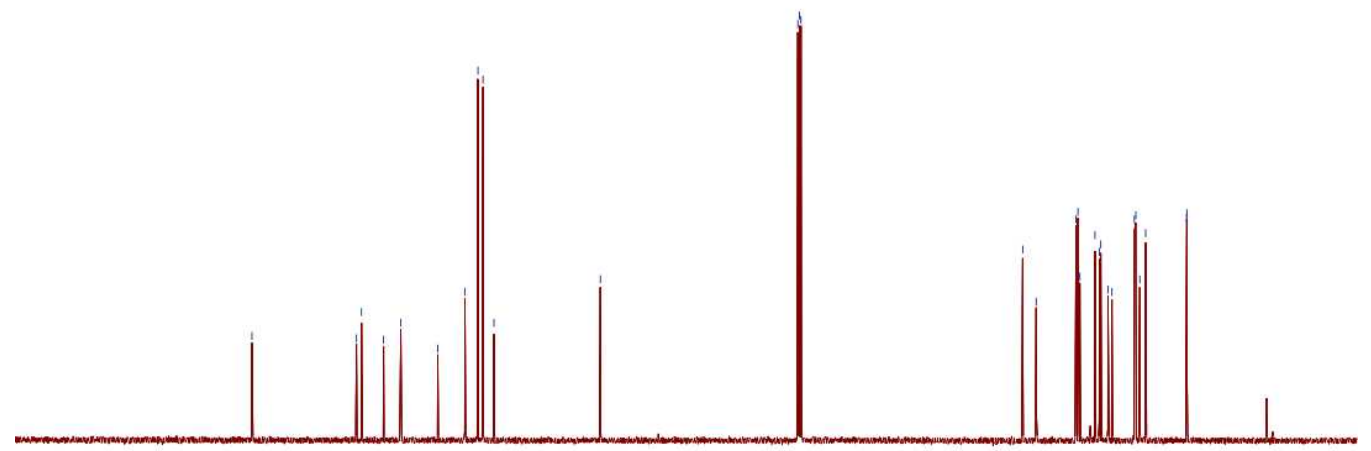

$\begin{array}{llllllllllllllllllllll}200 & 190 & 180 & 170 & 160 & 150 & 140 & 130 & 120 & 110 & \begin{array}{c}100 \\ \text { f1 }(\mathrm{ppm})\end{array} & 80 & 70 & 60 & 50 & 40 & 30 & 20 & 10 & 0 & -10\end{array}$ 


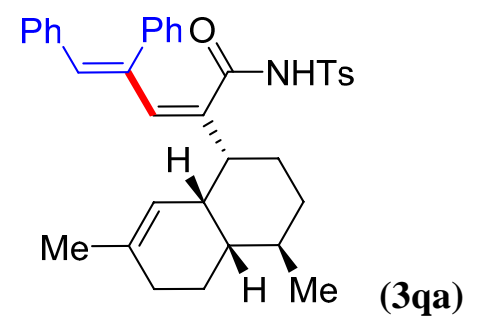

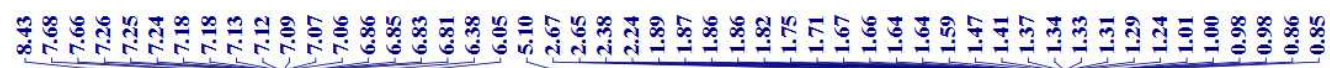
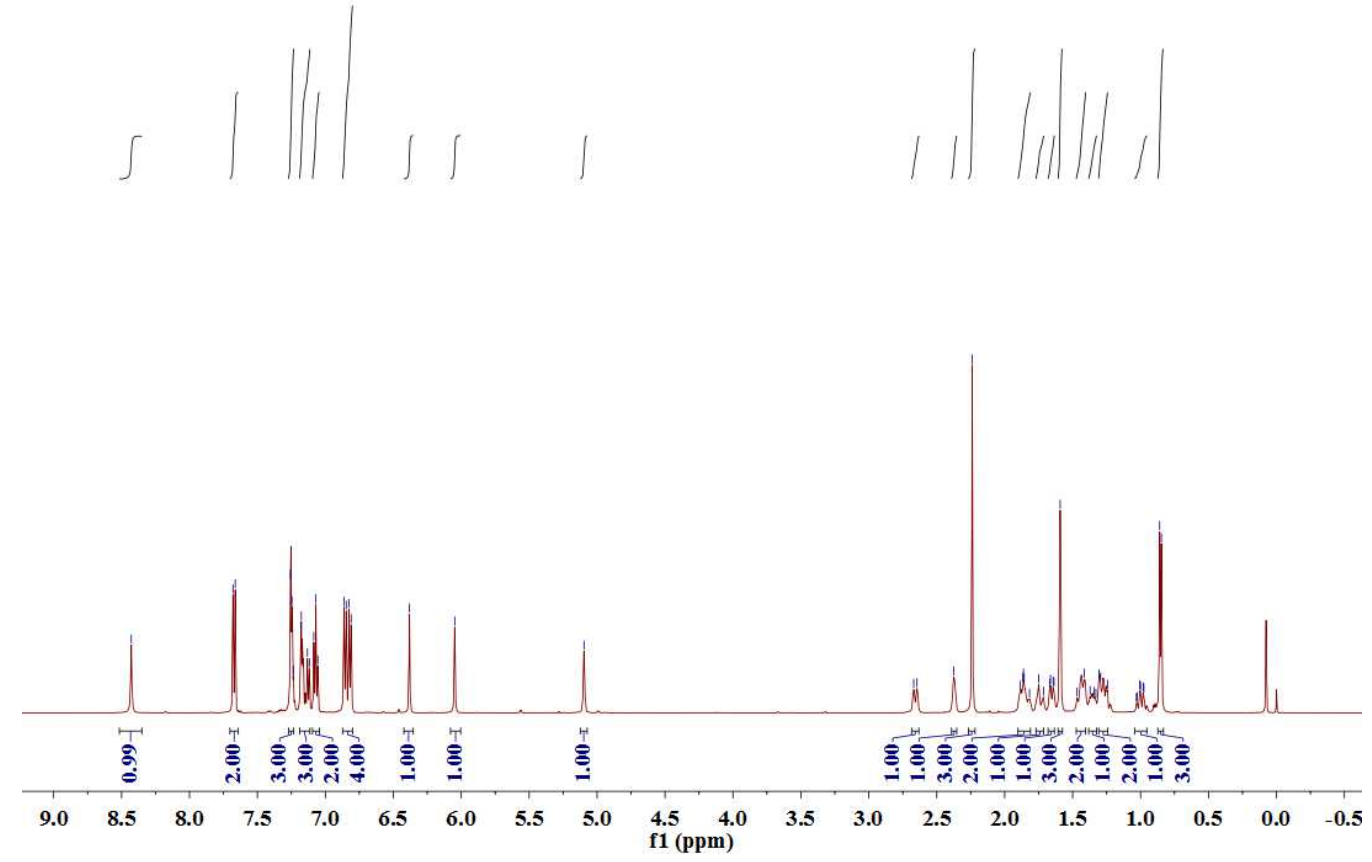

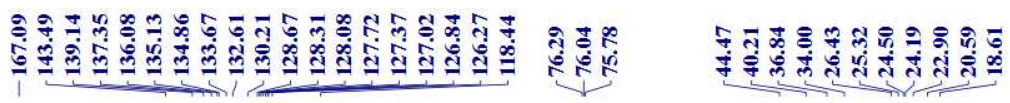

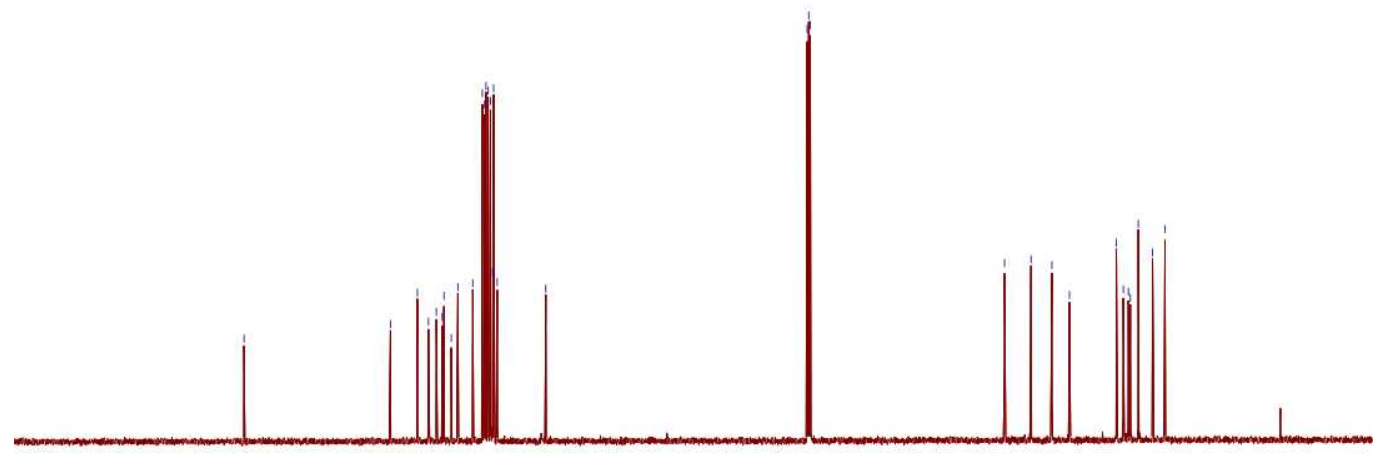

$\begin{array}{llllllllllllllllllllll}200 & 190 & 180 & 170 & 160 & 150 & 140 & 130 & 120 & 110 & 100 & 90 & 80 & 70 & 60 & 50 & 40 & 30 & 20 & 10 & 0 & -10\end{array}$ 
<smiles>C/C(=C/C(=C/P)c1ccccc1)C(=O)O</smiles>

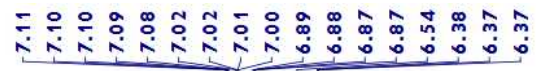
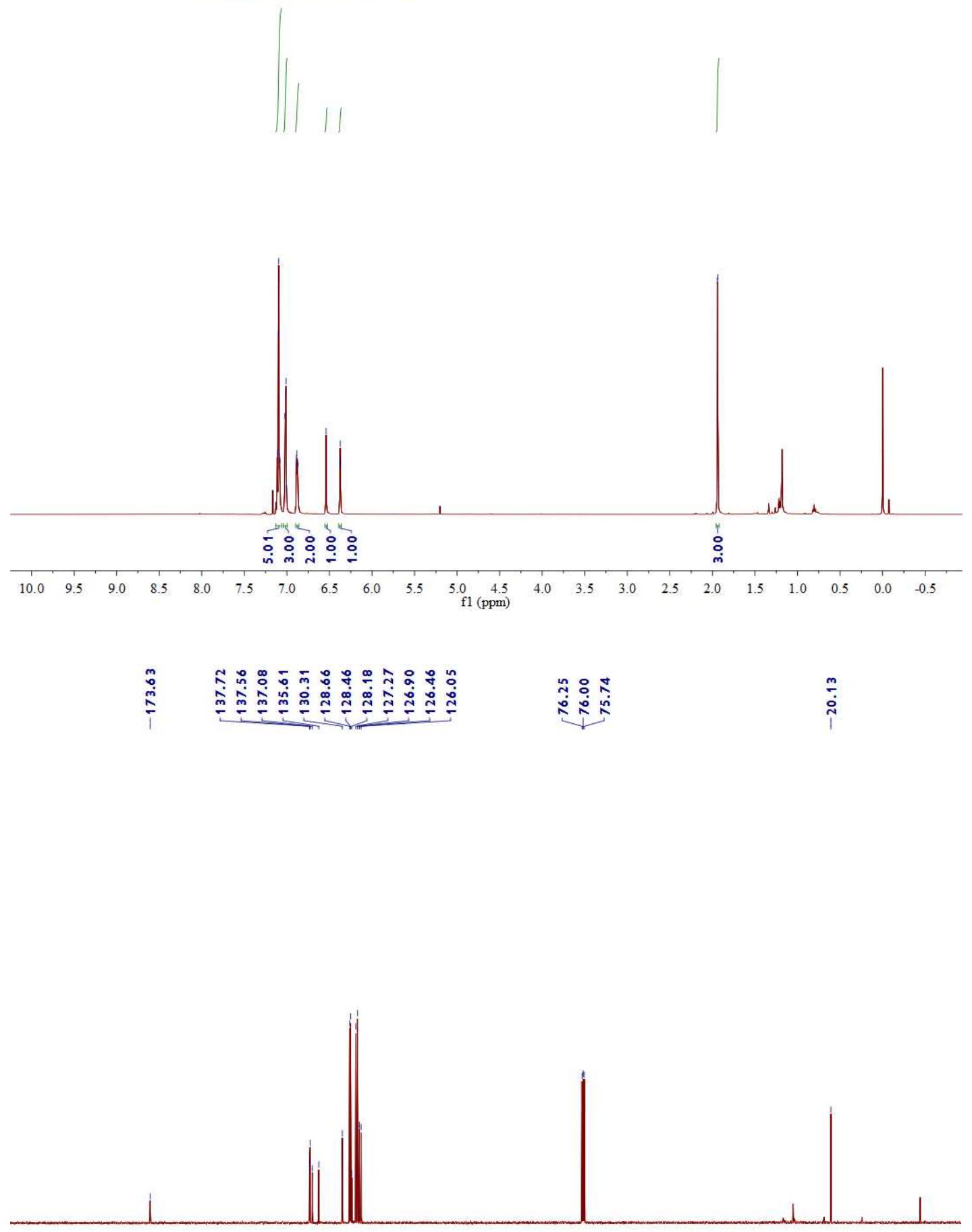

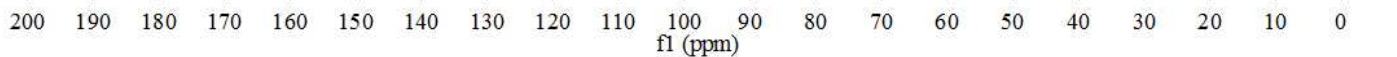


(3aa)

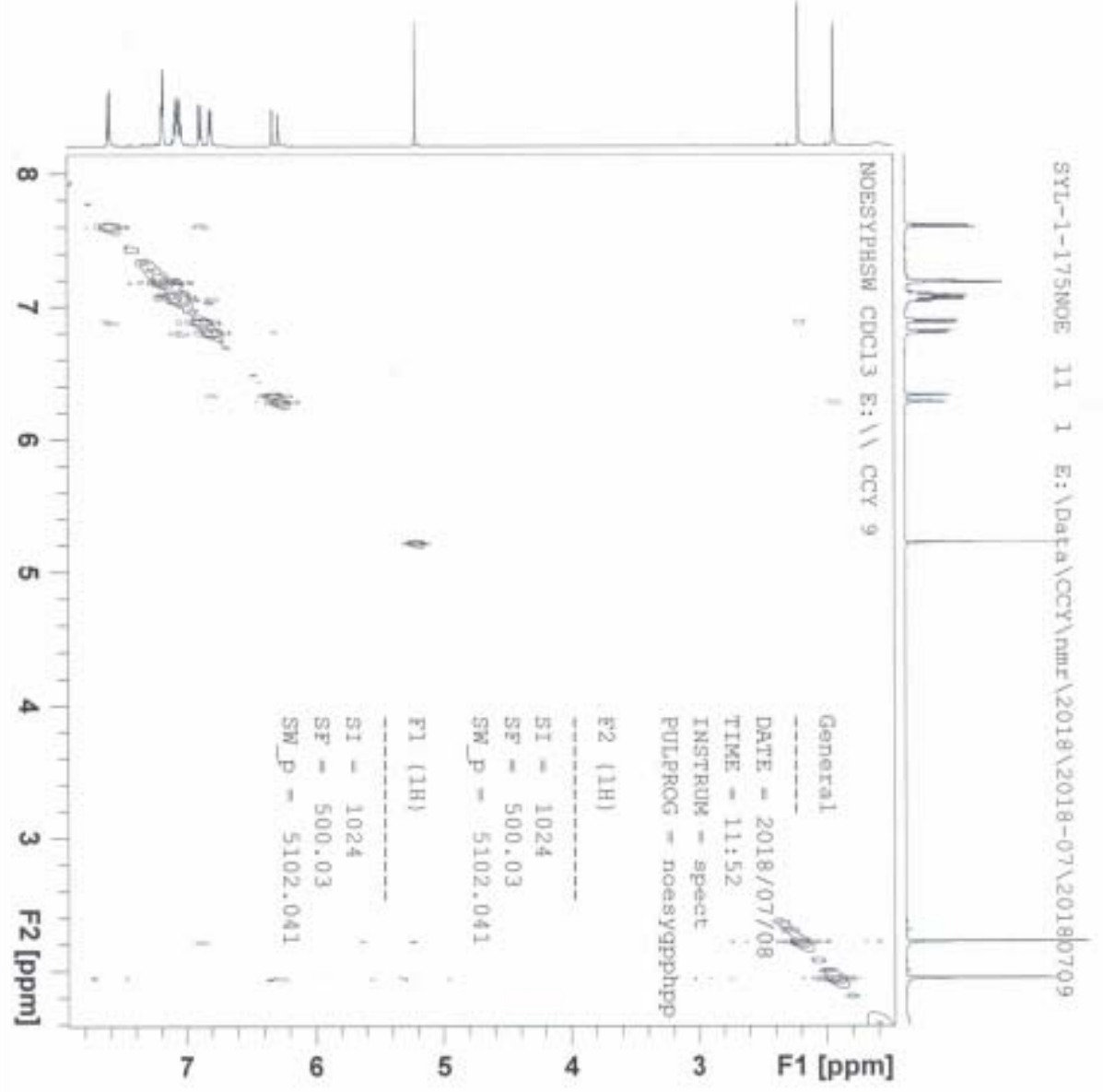

